\title{
UC-NRLF
}

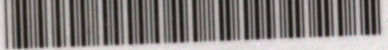

B $4250 \quad 115$ 


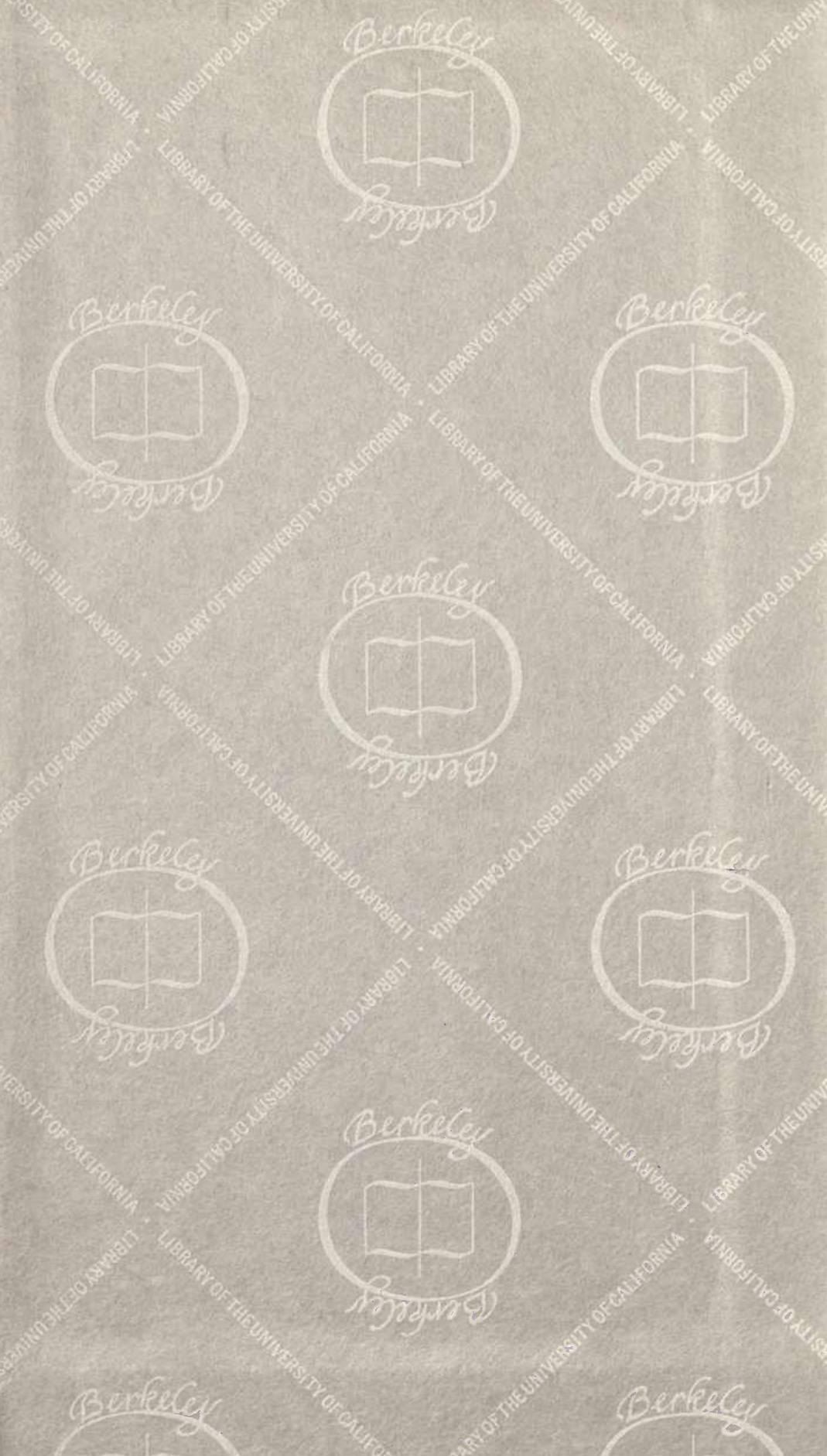




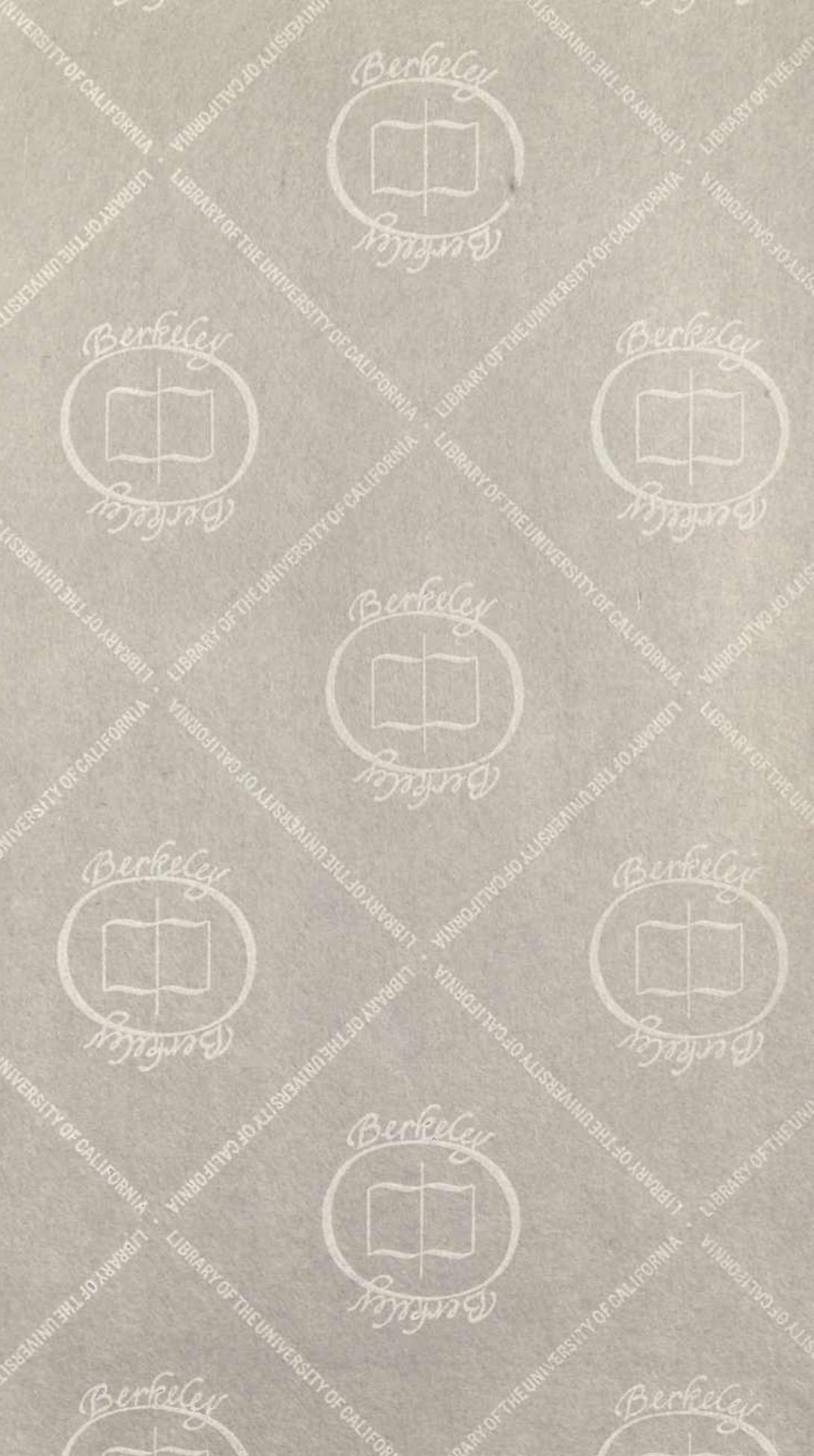






\section{THEORY OF SCREWS.}




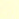




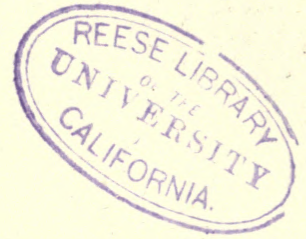




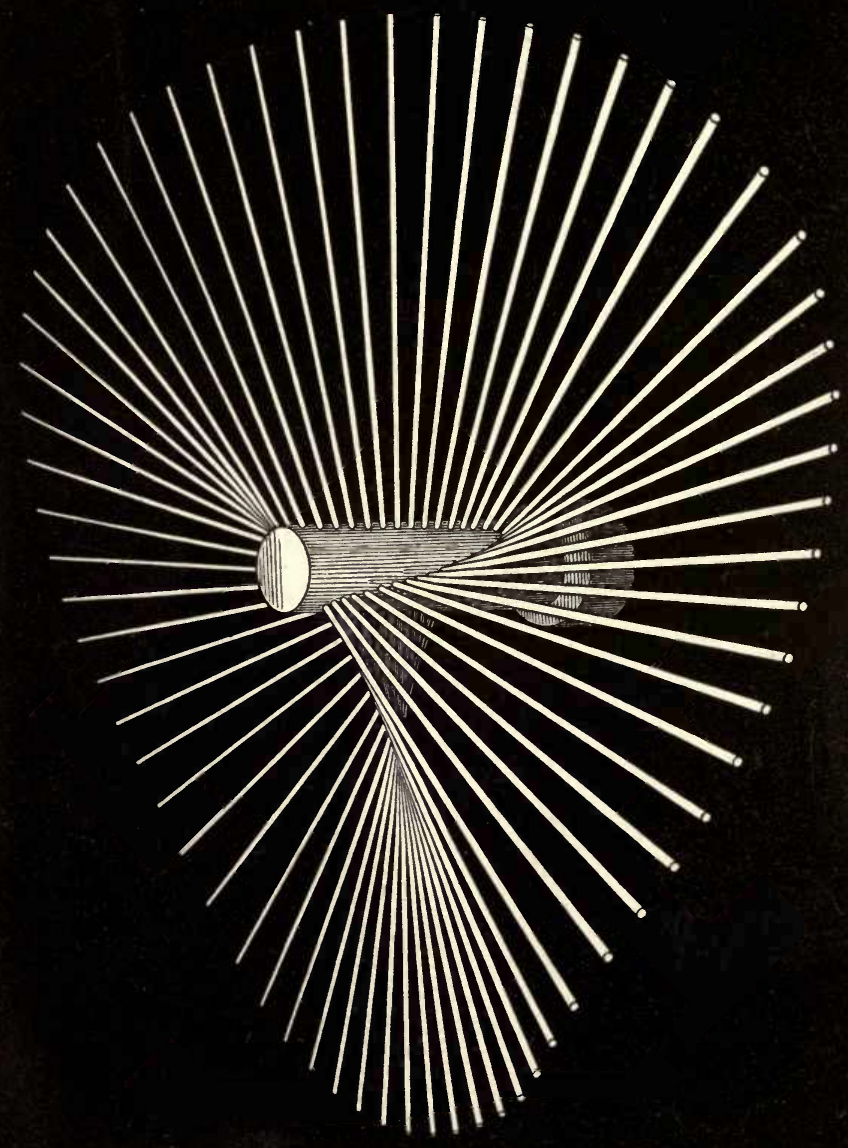

离 
THE

\section{THEORY OF SCREWS:}

A STUDY IN THE DYNAMICS OF A RIGID BODY.

BY

ROBERT STAWELL BALL, LL.D., F.R.S., ANDREWS' PROFESSOR OF ASTRONOMY IN THE UNIVERSITY OF DUBLIN, AND ROYAL ASTRONOMER OF IRELAND.
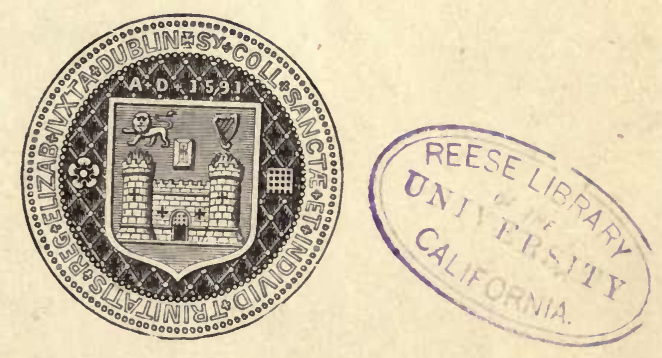

DUBLIN :

HODGES, FOSTER, AND CO., GRAFTON-STREET.

BOOKSELLERS TO THE UNIVERSITY.

1876 . 


$$
\begin{aligned}
& Q^{8^{41}} \\
& B^{3}
\end{aligned}
$$

DUBLIN :

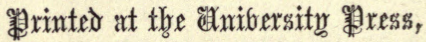
BY PONSONBY AND MURPHY.

46386 
TO MY FRIEND,

FREDERICK J. SIDNEY, LL.D.,

I DEDICATE THIS BOOK,

IN PLEASANT RECOLLECTION OF THE YEARS

DURING' 'WHIC̀H I WAS

A PROFESSOR AT THE ROYAL COLLEGE OF SCIENCE

FOR IRELAND. 


\section{CONTENTS.}

\section{INTRODUCTION.}

PAGE.

I. On the Canonical Form of a System of Forces applied to a Rigid Body, xvii II. On the Canonical Form of the Displacement of a Rigid Body, . xviii

\section{CHAPTER I.}

TWISTS AND WRENCHES.

Sect.

I. Definition of the word Screw, .

2. Definition of the word Twist, . . . . . . . . 3

3. Instantaneous Screws, . . . . . . . . . . 4

4. Definition of the word Wrench, . . . . . . . . ib.

5. Notation for Twists, Wrenches, and Twisting Motions, . . . 5

6. Restriction of the Forces, . . . . . . . . . 7

7 The Energy of Position, . . . . . . . . . . ib.

8. Theorem, . . . . . . . . . . . 8

9. Theorem, . . . . . . . . . . . . . . . . . . .

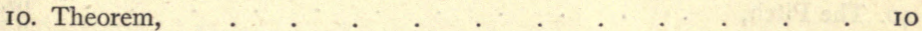

II. Concluding Remarks, . . . . . . . . . . . . II

\section{CHAPTER II.}

\section{THE CYLINDROID.}

12. Introduction,

13. On the Virtual Co-efficient of a Pair of Screws, 14. Symmetry of the Virtual Co-efficient, . . . . . . . . I3

I5. Composition of Twists and Wrenches, . . . . . . . ib.

I6. The Cylindroid, . . . . . . . . . . 14

17. General Property, . . . . . . . . . . 17

18. Particular Cases, . . . . . . . . . . 18

19. Form of the Cylindroid, . . . . . . . . . . . $\quad$. 19

20. The Pitch Conic, . . . . . . . . . . ib.

21. Summary, . . . . . . . . . . 20 


\section{CHAPTER III.}

RECIPROCAL SCREWS.

Sect.

PAGE.

22. Reciprocal Screws, :

23. Particula r Instances,

24. Screw Reciprocal to Cylindroid,

25. Reciprocal Cone,

26. Locus of a Screw Reciprocal to Four Screws, • • • . . 24

27. Screw Reciprocal to Five Screws, . • . • . . 25

28. Screw upon a Cylindroid Reciprocal to a Given Screw, . . $\quad 26$

29. Properties of the Cylindroid, . . . . . . . . ib.

\section{CHAPTER IV.}

\section{SCREW CO-ORDINATES.}

30. Introduction,

3 r. Intensities of the Components,

32. The Intensity of the Resultant,

33. Co-reciprocal Screws,

34. Co-ordinates of a Wfench,

35. The Work done in a Twist,

36. Screw Co-ordinates,

37. Identical Relation, .

38. Calculation of Co-ordinates,

39. The Virtual Coefficient, .

40. The Pitch,

41. Screw Reciprocal to Five Screws,

42. Co-ordinates of a Screw on a Cylindroid,

\section{CHAPTER V.}

\section{GENERAL CONSIDERATIONS ON THE EQUILIBRIUM OF A RIGID} BODY.

43. The Screw Complex,

44. Constraints, . .

45. Screw Reciprocal to a Complex, . . . . . . . ib.

46. The Reciprocal Screw Complex, . . . . . . . 40

47. Equilibrium, • • • • . . . . . . . 4 4

48. Reaction of Constraints, . . . . . . . . ib.

49. Parameters of a Screw Complex, . . . . . . 42

50. Applications of Co-ordinates, . . . . . . . . 43 


\section{CHAPTER VI.}

THE PRINCIPAL SCREWS OF INERTIA.

Sect.

51. Introduction,

PAGE.

$\cdot \cdot 46$

53. Impulsive Screws and Instantaneous Screws, . . . . . 47

54. Conjugate Screws of Inertia, . . . . . . . . 48

55. Determination of the Impulsive Screw, . . . . . 49

56. System of Conjugate Screws of Inertia, . . . . . . . ib.

57. Principal Screws of Inertia, . . . . . . . . 50

58. Kinetic Energy, . . . . . . . . . 52

59. Expression for Kinetic Energy, . . . . . . $\quad 54$

6o. Twist Velocity acquired by an Impulse, . . . . . . 55

61. The Kinetic Energy acquired by an Impulse, . . . . $\quad 56$

62. Free Body, . . . . . . . . . . ib.

63. Lemma, . . . . . . . . . . . 67

64. Euler's Theorem, . . . . . . . . . . ib.

65. Co-ordinates of a Screw belonging to a Screw Complex, . . $\quad 5^{8}$

66. The Reduced Wrench, . . . . . . . . 59

67. Co-ordinates of Impulsive and Instantaneous Screws, . . . 60

\section{CHAPTER VII.}

THE POTENTIAL ENERGY OF A DISPLACEMENT.

68. The Potential Energy of a Displacement, . . . . . 63

69. The Wrench evoked by Displacement, . . . . . . 64

70. Conjugate Screws of the Potential, . . . . . . . 66

71. Principal Screws of the Potential, . . . . . . . 68

72. Co-ordinates of the Wrench evoked by a Twist, . . . 69

73. Form of the Potential, . . . . . . . 71

\section{CHAPTER VIII.}

\section{HARMONIC SCREWS.}

74. Definition of an Harmonic Screw, . . . . - . 73

75. Equations of Motion, . . . . . . . . . . 76

76. Discussion of the Results, . . . . . . . 80

77. Remarks on Harmonic Screws, . . . . . . $\quad 82$ 


\section{CHAPTER IX.}

THE DYNAMICS OF A RIGID BODY WHICH HAS FREEDOM OF THE FIRST ORDER.

Sect.

PAGr.

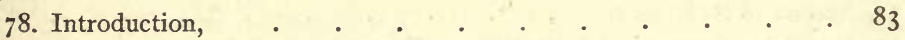

79. Screw Complex of the First Order, . . . . . . ib.

80. The Reciprocal Screw Complex, . . . . . . . 84

81. Equilibrium, . . . . . . . . . . . 86

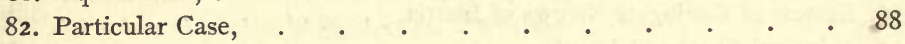

83. Impulsive Forces, . . . . . . . . . . . ib.

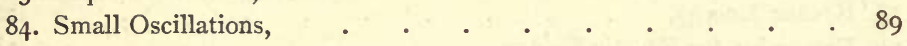

85. Property of Harmonic Screws, . . . • • . 90

\section{CHAPTER X.}

THE DYNAMICS OF A RIGID BODY WHICH HAS FREEDOM OF THE SECOND ORDER.

86. The Screw Complex of the Second Order, . . . . $\quad$ - 92

87. Applications of Screw Co-ordinates, . . . . . . ib.

88. Relation between Two Cylindroids, • • • • • . 93

89. Co-ordinates of Three Screws on a Cylindroid, . . . . 94

90. Screw Complex of the Fifth Order and Second Degree, . 95

91. Polar Screws, . . . . . . . . . . . . 96

92. Properties of Screws and their Polars, . . . . . . . . 98

93. Pitch Complex, . . . . . . . . . ib.

94. Screws on One Line, . . . . . . . . . . 101

95. Displacement of a Point, . . . . . . . . 102

96. Properties of the Pitch Conic, . . . . . . . 103

97. Equilibrium of a Body with Freedom of the Second Order, . . ib.

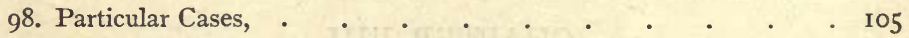

99. The Impulsive Cylindroid and the Instantaneous Cylindroid, . ib.

100. Reaction of Constraints, . . . . . . . . 107

ror. Principal Screws of Inertia, . . . . . . . . 108

102. The Ellipse of Inertia, . . . . . . . . 109

I03. The Ellipse of the Potential, . . . . . . . . III

I04. Harmonic Screws, . . . . . . . . . . II3

105. Exceptional Case, . . . . . . . . . . . ib.

I06. Reaction of Constraints, . . . . . . . . II4 


\section{CHAPTER XI.}

THE DYNAMICS OF A RIGID BODY WHICH HAS FREEDOM OF THE THIRD ORDER.

Sвст.

107. Introduction, . PAGB. 108. Screw Complex of the Third Order, 108. Screw Complex of the Third Order, . • . II7 I09. The Reciprocal Screw Complex, . . . . . . . I18 I Io. Distribution of the Screws, . . . . . . . . . ib. 11 I. The Pitch Quadric, . . . . . . . . . . . II9 112. Screws through a Given Point, . . . . . . 122 1 3. Screws of the Complex parallel to a Plane, . . . . 125 114. Determination of the Cylindroid, . . . . . . . 126 115. Miscellaneous Remarks, . . . . . . . . 128 I16. Virtual Co-efficients, . . . . . . . . . 130 II7. Four Screws of the Screw Complex, . . . . . . ib. I 18. Equilibrium of Four Forces applied to a Rigid Body, . . . I3I 119. The Ellipsoid of Inertia, . . . . . . . . 133 1 20. The Principal Screws of Inertia, . . . . . . 134 121. Lemma, . . . . . . . . . . . 135 122. Relation between the Impulsive Screw and the Instantaneous Screw, ib. 123. Kinetic Energy acquired by an Impulse, . . . . . . . 136 124. Reaction of the Constraints, . . . . . . . . 138 125. Impulsive Screw is Indeterminate, . . . . . . ib. 126. Ellipsoid of the Potential, . . . . . . . 139 127. The Principal Screws of the Potential, . . . . . 140 128. Wrench evoked by Displacement, . . . . . . . ib. 129. Harmonic Screws, . . . . . . . . . 141 130. Oscillations of a Rigid Body about a Fixed Point, _ . . 142

\section{CHAPTER XII.}

THE DYNAMICS OF A RIGID BODY WHICH HAS FREEDOM OF THE FOURTH ORDER.

131. Screw Complex of the Fourth Order, . . . . . . 146

132. Screws Parallel to a Given Line, . . . . . . . . ib.

133. Screws in a Plane, . . . . . . . . . ib.

134. Property of the Pitches of Six Co-reciprocals, . . . . 147

135. Another Proof, . . . . . . . . 149

136. Property of the Pitches of $n$ Co-reciprocals, . . . . 149

137. Special Screw of the Complex, . . . . . . 150

138. Particular Case, . . . . . . . . . . ib.

139. Statics, . . . . . . . . . . . . ib. 
Chapter XII.-continued.

Secr.

PAGE.

140. Equilibrium of Five Forces, . . . . . . . . 151

I4 r. Problem, . . . . . . . . . . . 152

142. Impulsive Screws and Instantaneous Screws, _ . . . . . ib.

143. Principal Screws of Inertia, . . . . . • . . 153

144. Application of Euler's Theorem, . . . . . . . 154

145. General Remarks, . . . . . . . . . 155

146. The Screw Complex of the $(n-1)^{\text {th }}$ Order and Second Degree, . 156

147. Polar Screws, . . . . . . . . . . 157

148. Kinetic Complex, . . . . . . . . . . 158

149. The Potential Complex, . . . . . . . . 160

150. Harmonic Screws, . . . . . . . . . . ib.

\section{CHAPTER XIII.}

THE DYNAMICS OF A RIGID BODY WHICH HAS FREEDOM OF THE FIFTH ORDER.

151. Screw Reciprocal to Five Screws, . . . . . . . 16i

152. Definition of the Sexiant, . . . . . . . . 163

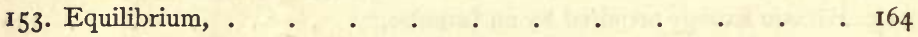

154. Impulsive Screws and Instantaneous Screws, . . . . . 166

155. Analytical Investigation, . . . • . . . . 167

156. Principal Screws of Inertia, . . . . . . . 168

\section{CHAPTER XIV.}

THE DYNAMICS OF A RIGID BODY WHICH HAS FREEDOM OF THE SIXTH ORDER.

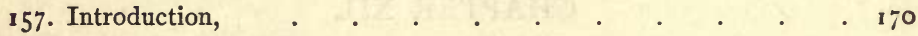

158. Impulsive Screws, . . . . . . . . . . ib.

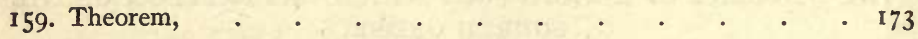

160. Theorem, . . . . . . . . . . . ib.

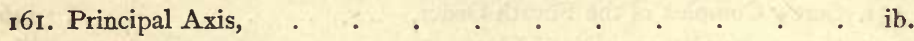

162. Harmonic Screws, . . . . . . . . . 174 


\section{INDEX TO DEFINITIONS.}

The Numerals refer to the Numbers of the Paragraphs.

Sect.

Amplitude of a Twist

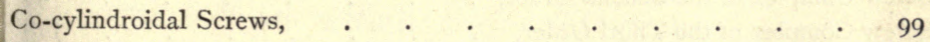

Conjugate Screws of Inertia, . $\quad . \quad$. $\quad . \quad . \quad . \quad .48$

Conjugate Screws of the Potential, . . . . . . . . 66

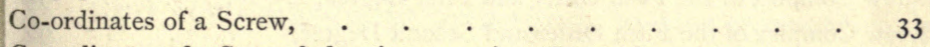

Co-ordinates of a Screw belonging to a given Screw Complex, . $\quad 59$

Co-ordinates of a Twist, . . . . . . . . . . . 32

Co-ordinates of a Wrench, . . . . . . . . . ib.

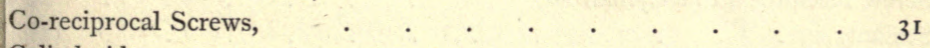

Cylindroid, $\quad . \quad . \quad . \quad . \quad . \quad . \quad . \quad . \quad . \quad . \quad . \quad . \quad . \quad . \quad{ }_{15}$

Ellipse of Inertia, . . . . . . . . . . . . . I I0

Ellipse of the Potential, . . . . . . . . . . 112

Ellipsoid of Inertia, . $\quad . \quad . \quad . \quad . \quad . \quad . \quad . \quad . \quad . \quad$ I $\quad . \quad$.

Ellipsoid of the Potential, . $\quad . \quad$. $\quad . \quad . \quad . \quad . \quad . \quad . \quad 140$

Freedom of the $n^{\text {th }}$ Order, . . . . . . . . . . . . 43

Harmonic Screws, $. \quad . \quad . \quad . \quad . \quad . \quad . \quad . \quad . \quad .74$

Impulsive Cylindroid, . $\quad . \quad$. $\quad . \quad . \quad . \quad . \quad . \quad . \quad . \quad 106$

Impulsive Screw, $\quad . \quad . \quad . \quad . \quad . \quad . \quad . \quad . \quad . \quad . \quad .47$

Impulsive Wrench, . $\quad . \quad . \quad . \quad . \quad . \quad . \quad . \quad . \quad .45$

Instantaneous Cylindroid, . $\quad . \quad . \quad . \quad . \quad . \quad . \quad . \quad . \quad 106$

Instantaneous Screw, . . . . . . . . . . . . . 4

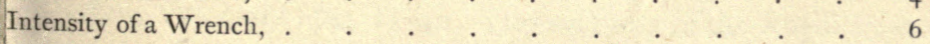

Kinetic Screw Complex, . . . . . . . . . . . ${ }^{159}$ 
Sect.

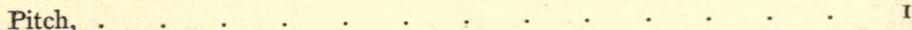

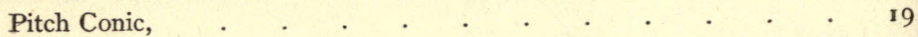

Pitch Quadric, . . . . . . . . . . . . . 1 IJ9

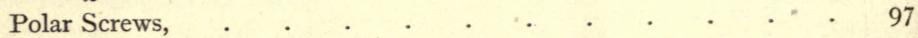

Potential Screw Complex, . . . . . . . . . 149

Principal Screws of Inertia, . . . . . . . . . . 45

Principal Screws of the Potential, . . . . . . . 68

Reciprocal Plane, . . . . . . . . . . . 122

Reciprocal Screws, . . . . . . . . . . 2 I

Reciprocal Screw Complex, . . . . . . . . . 41

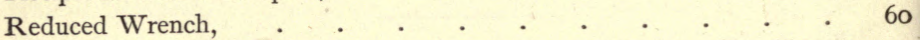

Resultant Twist, . $\quad . \quad . \quad . \quad . \quad . \quad . \quad . \quad .33$

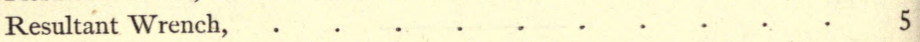

Screw, . . . . . . . . . . . . 3

Screw Complex, . . . . . . . . . . . 38

Screw Complex of the First Order, . . . . . . . . 83

Screw Complex of the Second Order, . $\quad$. $\quad$. . . . . . 92

Screw Complex of the Third Order, . . . . . . $\quad 118$

Screw Complex of the Fourth Order, . . . . . . . 147

Screw Complex of the Fifth Order and First Degree, . _ . . . 95

Screw Complex of the Fifth Order and Second Degree, . . . 96

Screw Complex of the Sixth Order, . . . . . . . 157

Screw Complex of the $(n-1)^{\text {th }}$ Order and Second Degree, . . . $\quad{ }_{1} 5^{8}$

Screw Reciprocal to a Cylindroid, . . . . . . 22

Sexiant, . . . . . . . . . . . 151

Twist, . . . . . . 3

Twisting Motion, . . . . . . . . . . . 6

Twist Velocity, . . . . . . . . . . . . . ib.

Virtual Co-efficient, . . . . . . . . . . . . 13

Wrench, . . . . . . . . . . . . . 4

Wrench evoked by Displacement, . . . . . . . 64 


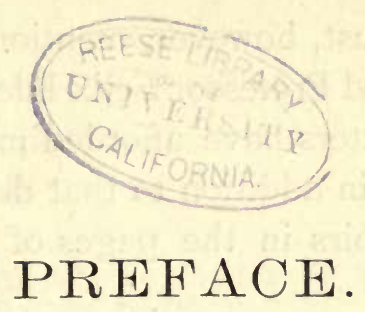

THE Theory presented in the following pages was first sketched by the author in a Paper* communicated to the Royal Irish Academy on the 1 3 th of November, 1871. This Paper was followed by others, $\uparrow$ in which the subject was more fully developed. The entire Theory has been re-written, and systematically arranged, in the present volume.

We owe to the geometrical ability of Poinsot and Chasles the two fundamental theorems from which this subject takes its rise. To the labours of Plücker, and his school, we are indebted for the theory of linear geometry, which receives a physical interpretation by the Theory of Screws.

References are made in the foot notes, and more fully in the Appendix, to various authors whose writings are connected with the subject discussed in

* Transactions of the Royal Irish Academy, Vol. xxv., pp. 157-217.

† Philosophical Transactions of the Royal Society of London, Vol. clxiv., pp. 15-40. Transactions of the Royal Irish Academy, Vol. xxv., pp. 295-327. 
this book. I must, however, mention specially the name of my friend Professor Felix Klein, of Munich, whose private letters have afforded me much valuable information, in addition to that derived from his instructive memoirs in the pages of the Mathematische Annalen.

To my friends, Rev. Professor Townsend, F. R. S., Professor Everett, Dr. Tarleton, F. T. C. D., Professor Niven, and Professor Casey, F. R. S., my thanks are due for many useful suggestions and a little friendly criticism, while to the two first-named I am further indebted for their trouble in looking over the proof-sheets in their passage through the press.

My grateful thanks are also due to the Board of Trinity College for a liberal contribution towards the expenses of publication; and to the Council of the Royal Irish Academy for kind permission to use the wood engravings which illustrated the original Papers published in their Transactions.

\section{ROBERT S. BALL.}

The Observatory, Dunsink, Co. Dublin, 31 st December, 1875 . 


\section{INTRODUCTION.}

THE Theory of Screws is founded upon two well-known theorems. One relates to a system of forces acting on a rigid body; while the other relates to the displacement of a rigid body. Although these two theorems are to be found in many treatises on mechanics, yet a discussion of them here, so far as they are necessary for our purpose, may be useful.

\section{I.-ON THE REDUCTION OF A SYSTEM OF FORCES APPLIED}

TO A RIGID BODY TO ITS CANONICAL FORM.

The Canonical Form.-It has been discovered by Poinsot* that any system of forces which act upon a rigid body can be replaced by a single force, and a couple in a plane perpendicular to the force. Thus a force, and a couple in a plane perpendicular to the force, constitute what may be called the canonical form of a system of forces applied to a rigid body.

It is easily seen that all the forces acting upon a rigid body may, by transference to an arbitrary origin, be compounded into a force acting at the origin, and a couple. Wherever the origin be taken, the magnitude and direc-

* See Appendix I. 
tion of the force are both manifestly invariable; but this is not the case either with the moment of the couple or the aspect of its plane.

The origin, however, can be always so selected that the plane of the couple shall be perpendicular to the direction of the force. For at any origin the couple can be resolved into two couples, one in a plane containing the force, and the other in the plane perpendicular to the force. The first component can be compounded with the force, the effect being merely to transfer the force to a parallel position; thus the entire system is reduced to a force, and a couple in a plane perpendicular to it.

The Canonical Form is Unique.-It is very important to observe that there is only one straight line which possesses the property that a force along this line, and a couple in a plane perpendicular to the line, is equivalent to the given system of forces. Suppose two lines possessed the property, then if the force and couple belonging to one were reversed, they must destroy the force and couple belonging to the other. But the two straight lines must be parallel, since each must be parallel to the resultant of all the forces supposed to act at a point, and the forces acting along these must be equal and opposite. The two forces would therefore form a couple in a plane perpendicular to that of the couple which is found by compounding the two original couples. We should then have two couples in perpendicular planes destroying each other, which is manifestly impossible.

We thus see that any system of forces applied to a rigid body can be made to assume an extremely simple form, in which no arbitrary element is involved. 
II.-ON THE REDUCTION OF THE DISPLACEMENT OF A RIGID BODY TO ITS CANONICAL FORM.

Problem.-Two positions of a rigid body being given, there are an infinite variety of movements by which the body can be transferred from one of these positions to the other. It has been discovered by Chasles* that among these movements there is one of unparalleled simplicity. The demonstration of this theorem is the object of the present section.

The Composition of Rotations about Intersecting Axes.Suppose a body receive a small rotation through an angle $\boldsymbol{a}$ about a certain axis, and another small rotation through an angle $\beta$ around a second axis intersecting the former one; then the position ultimately attained could have been reached by a single rotation from the initial position about an axis appropriately chosen.

Let $O A$ and $O B$ (Fig. I) represent the directions of the given axes, while their lengths are proportional to the angles $a$ and $\beta$, the directions of the rotations being such that if an ordinary screw were placed with its head at $O$, and its axis along $O A$, then the direction of the rotation which would make the screw advance from $O$ is the direction of the rotation

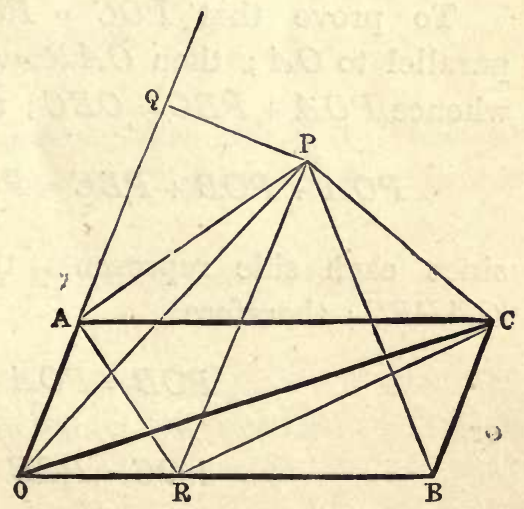

Fig. I. 
indicated by $O A$, with a similar convention for $O B$. Completing the parallelogram $O A C B$, we shall prove that a rotation around $O C$, through an angle proportional to the length $O C$, will have precisely the same effect as the two given rotations.

Consider any point $P$ of the body which lies in the plane of the axes. The rotation $O A$ will depress the point $P$ below the plane of the paper along the normal to a small distance which is proportional to the product of $O A$, and the perpendicular $P Q$; that is, proportional to the area of the triangle $P O A$. In the same way the rotation around $O B$ will raise $P$ above the plane of the paper to a distance which is proportional to the area of $P O B$. The joint effect will be to raise $P$ to a distance above the paper proportional to the difference between the areas of the triangle $P O B$ and $P O A$. - that is, to the area of $P O C$; but this is precisely the same effect as would be produced by a rotation around $O C$ through an angle proportional to $O C$.

To prove that $P O C=P O B-P O A$ : draw $P R$ parallel to $O A$; then $O A R=O A P$, and $B R C=B P C$, whence $P O A+P B C=O B C$; also we have

$$
P O A+P O B+P B C=P O A+P O C+O B C,
$$

since each side represents the area of the figure $O A P B C$; therefore

$$
P O B=P O A+P O C
$$

or

$$
P O C=P O B-P O A \text {. }
$$

The rotation around $O C$ must, therefore, produce precisely the same effect on every point in the plane as is produced by the joint effect of the rotations around $O A$ and $O B$. 
and hence it follows, that the two rotations about $O A$ and $O B$ can be replaced by the single one about $O C$.

The correspondence between the solution of this problem and the principle embodied in the parallelogram of force should be noticed. We see that rotations about intersecting axes are compounded by the same rules as intersecting forces.

Composition of Rotations about Parallel Axes.-We shall now consider the case in which the two axes $A$ and $B$, about which the body receives small rotations $a$ and $\beta$, are parallel. Divide the perpendicular distance $d$ between the parallel axes $A$ and $B$ in the inverse proportion of $a$ and $\beta$, and draw a line $C$ parallel to $A$ and $B$ through the point thus obtained. We shall show that a rotation around $C$ through an angle $a+\beta$ will be precisely equivalent to the two given rotations. For consider any point $P$ in the plane at a perpendicular distance $x$ from $C$. Then the distances of $P$ from $A$ and from $B$ are respectively

$$
x+d \frac{\beta}{a+\beta} \text {, and } x-d \frac{a}{a+\beta} .
$$

The effect of the rotations about $A$ and $B$ will, therefore, be to raise $P$ above the plane of $A$ and $B$ to an amount

$$
a\left(x+d \frac{\beta}{\alpha+\beta}\right)+\beta\left(x-d \frac{a}{a+\beta}\right)=(\alpha+\beta) x ;
$$

but rotation about $C$ through an angle $a+\beta$ would have had precisely the same effect, and the same will be true for every other point in the plane besides $P$.

We thus see that rotations about parallel axes are compounded by exactly the same lawes as parallel forces.

Translations.-The rule for the composition of parallel rotations would not apply if the two rotations were 
equal and opposite. We proceed to consider this case. Let the angle of rotation be $\boldsymbol{a}$, the axes $A$ and $B$, and their distance $d$. Let $x$ be the distance of any point $P$ from $A$; then the rotation about $A$ elevates $P$ above the plane of $A$ and $B$ to a distance $a x$. The rotation around $B$ depresses $P$ below the plane of $A$ and $B$ to a distance $a(x+d)$. The net result, therefore, is that $P$ is depressed below the plane of $A$ and $B$ to a distance $a d$. Now it is remarkable that this result is independent of the position of $P$ in the plane of $A$ and $B$; consequently all points in the plane are moved through equal distances, and thus we have the important result that a pair of equal parallel and opposite rotations are equivalent to a translation in the direction perpendicular to the plane of the axes, and through an interval proportional to the distance between them.

The converse of this result is also of great importance-namely, that a translation can always be decomposed into a pair of equal parallel but opposite rotations, in a plane perpendicular to the direction of the translation.

Composition of a Rotation with a Translation Perpendicular to the Axis of Rotation.-The translation may be resolved into a pair of equal parallel and opposite rotations in a plane which contains the given axis of rotation. This couple of rotations may be compounded with the given rotation in precisely the same way as a couple is compounded with a force in the same plane. It follows that the result of compounding a rotation with a translation perpendicular thereto is merely to transfer the rotation to a parallel position, without altering its magnitude.

Displacement of a Rigid Body about a Fixed Point.A rigid body is supposed to be free to turn around a fixed point $O$ in every way. If we fix our attention on 
any two adjacent positions of the body, we shall prove that it is possible for the body to be moved from one of these positions to the other by simple rotation round one axis. Describe a sphere round $O$ as centre, and let $P, Q$ be the positions of two points on the body of the sphere in the first position, and $P^{\prime}, Q^{\prime}$ the positions of the same points (still on the sphere of course) in the second position; a plane can be drawn, which shall bisect the angle $P O P^{\prime}$, and also be perpendicular to the line $P P^{\prime}$. By suitable rotation around any axis lying in this plane and passing through $O, P$ can be made to coincide with $P^{\prime}$. The next step is to rotate the body around the axis $O P$ in its new position until $Q$ is brought to $Q$, which is always possible, since by hypothesis $P Q=P^{\prime} Q^{\prime}$; thus by two rotations the desired change has been accomplished. But the two rotations can be compounded into one, and therefore the entire change may be produced by one rotation.

This proposition is also true, whatever be the magnitude of the displacements; but the proof we have given only applies to the small displacements with which we are concerned.

Reduction of any Displacement of a Rigid Body to a Rotation and a Translation.-Let $P, Q, R$ be three points of the body in the first position, and $P^{\prime}, Q, R^{\prime}$ the three positions assumed by these points after the body has been displaced. By a translation the body may be moved so that $P$ coincides with $P^{\prime}$, and then by a rotation the points $Q$ and $R$ may be brought to coincide with $Q$ and $R^{\prime}$. Thus by the combination of a rotation, and a translation, the desired change can be effected.

The Canonical Form.-In general the direction of the translation will be inclined to the axis of the rotation; but an equivalent rotation and translation can be always found in which this is not the case. 
Resolve the translation into two components-one parallel to the axis of rotation, and the other perpendicular thereto. The component perpendicular to the axis of rotation will have merely the effect of transferring the rotation into a parallel position. Thus the canonical form of the displacement of a rigid body consists of a rotation about an axis combined with a translation parallel to that axis.*

The Canonical Form is Unique.-It is easily seen that there is only one axis by rotation about which, and translation parallel to which, the rigid body can be brought from one given position to another given position; for suppose there were two axes $P$ and $Q$, which possessed this property, then by the movement about $P$, all the points of the body originally on the line $P$ continue thereon; but it cannot be true for any other line that all the points of the body originally on that line continue thereon after the displacement. Yet this would have to be true for $Q$, if by rotation around $Q$ and translation parallel thereto, the desired change could be effected. We thus see that the displacement of a rigid body can be made to assume an extremely simple form, in which no arbitrary element is involved.

* For another proof of Chasles' theorem by Professor Crofton, F. R. S., see Proceedings of the London Math, Soc., Vol, v., p. 25. 


\section{THEORY OF SCREWS.}

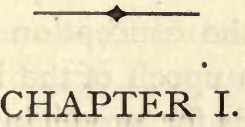

TWISTS AND WRENCHES.

$\S$ I. Definition of the word screw.-The direct problem offered by the Dynamics of a Rigid Body may be thus stated. To determine at any instant the position of a rigid body subjected to certain constraints and acted upon by certain forces. We may first inquire as to the manner in which the solution of any such problem ought to be presented. Adopting one position of the body as a standard of reference, a complete solution of the problem must provide us with the means of deriving the position at any subsequent epoch from the standard position. We are thus led to inquire into the most natural method of specifying one position of a body with respect to another.

To make our course plain let us consider the case of a mathematical point. To define the position of the point $P$ with reference to a standard point $A$, there can be no more natural method than to indicate the straight line along which it would be necessary for a particle to travel from $A$ in order to arrive at $P$, as well as the length of the journey. Now, there is an analogous method of defining the position of a rigid body with reference to a 
certain standard position. We can have a movement prescribed by which the body can be brought from the standard position to the sought position. We have seen, in the Introduction, that there is one preeminently simple movement which will always answer. A certain axis can be found, such that if the body be rotated around this axis through a certain angle, and translated parallel to the axis for a certain distance, the desired movement will be effected.

It will simplify the conception of the movement to suppose, that at each epoch of the interval of time occupied by the operations for producing the change of position, the angle of rotation bears to the final angle of rotation, the same ratio which the corresponding translation bears to the final translation. Under these circumstances the motion of the body is precisely the same as if it were rigidly attached to the nut of a screw (in the ordinary sense of the word), which had an appropriate position in space, and an appropriate number of threads to the inch.

In order to express, in a scientific manner, the relation between the rotation and the translation in the movement of a nut upon a screw, we give to the word pitch a special meaning. We define the pitch to be the rectilinear distance which the nut moves along the screw when the nut is rotated through the angular unit of circular measure. The pitch is thus a linear magnitude. The advantage of this convention is, that the rectilinear distance through which the nut moves when rotated through a given angle is simply the product of the pitch of the screw, and the circular measure of the angle.

It will presently appear that screws have a dynamical significance, which is of parallel importance to their kinematical properties. For this reason we attach a somewhat abstract sense to the word, by defining a screw 
to be a straight line in space with which a definite lnear magnitude, termed the pitch, is associated.

We shall often denote a screw by a symbol, and then usually by a small Greek letter. With reference to these symbols, a caution may be necessary. If, for example, a screw be denoted by $a$, then $a$ is not an algebraic quantity, and cannot occur in an algebraic equation. It is a symbol which denotes all that is included in the conception of a screw, and requires five quantities for its specification, because four quantities are required to determine a straight line, and the pitch must be specified by a fifth. It will often be convenient to denote the pitch by a symbol, which is derived from the symbol employed to denote the screw to which the pitch belongs. The pitch of a screw can be represented by appending to the letter $p$ a suffix denoting the screw; thus, $p_{a}$ is the pitch of $a$. The symbol $p_{a}$ represents, in fact, a certain number of millimetres, or inches.

$\S 2$. Definition of the word Twist.-We have now to define the very important use to be made of the word twist. A body is said to receive a twist about a screw when it is rotated about the screw, while it is at the same time translated parallel to the screw, through a distance equal to the product of the pitch and the circular measure of the angle of rotation; hence,

The canonical form to which the displacement of a rigid body can be reduced is a twist about a screw.

If a body receive several twists in succession, then the position finally attained could have been reached in a single twist, which is called the resultant twist.

Although we have described the twist as a compound movement, yet in the present method of studying mechanics it is essential to consider the twist as one homogeneous quantity. Nor is there anything unnatural in such a supposition. Everyone will admit that the rela- 
tion between two positions of a point is most simply presented by associating the purely metric element of length with the purely geometrical conception of a directed straight line. In like manner the relation of two positions of a rigid body can be most simply presented by associating a purely metric element with the purely geometrical conception of a screw, which is merely a straight line, with direction, situation, and pitch.*

$\S 3$. Instantaneous Screws. - Whatever be the movement of a rigid body, it is at every instant twisting about a screw. For the movement of the body when passing from one position to another position indefinitely adjacent, is indistinguishable from the twist about an appropropriately chosen screw by which the same displacement could be effected. The screw about which the body is twisting at any instant is termed the instantaneous screw.

$\S 4$. Definition of the word Wrench.-It has been proved in the Introduction, that the canonical form of a system of forces acting upon a rigid body consists of a force and a couple whose plane is perpendicular to the force. We now introduce the word wrench, to denote a force and a couple in a plane perpendicular to the force. The quotient obtained by dividing the moment of the couple by the force is a linear magnitude. Everything, therefore, which could be specified about a wrench is determined (if the force be given in magnitude), when the position of a straight line is assigned as the direction of the force, and a linear magnitude is assigned as the quo-

* Those acquainted with the language of the Quaternions, invented by the late Sir W. R. Hamilton, will perceive that a twist bears the same relation to a rigid body which a vector does to a point ; each just expresses what is necessary to transfer the corresponding object from one given position to another. 
tient just referred to. Remembering the definition of a screw, $(\S \mathrm{I})$, we may use the phrase, wrench on a screw, meaning thereby, a force directed along the screw and a couple in a plane perpendicular to the screw, the moment of the couple being equal to the product of the force and the pitch of the screw. Hence, we may state, that

The canonical form to which all the forces acting on a rigid body can be reduced is a wrench on a screw.

If a rigid body be acted upon by several wrenches, then these wrenches could be replaced by one wrench which is called the resultant rurench.

§5. Notation for Twists, Wrenches, and Twisting Motions.- A twist about a screw a requires six algebraic quantities for its complete specification, and of these, five are required to specify the screw $a$. The sixth, or metric quantity, which is called the AMPLITUDE OF THE TWIST, and is denoted by $\boldsymbol{a}^{\prime}$, expresses the angle of that rotation which, when united with a translation, constitutes the entire twist.

The distance of the translation is the product of the amplitude of the twist and the pitch of the screw, or in symbols $a^{\prime} p_{a}$.

If the pitch be positive (negative), the direction of the translation portion of the twist bears the same relation to the direction of the rotation portion of the twist as the direction of the translation of a nut on an ordinary righthanded (left-handed) screw bears to the direction of the rotation of the nut.

If the pitch be zero, the twist reduces to a pure rotation around $\boldsymbol{a}$. If the pitch be infinite, then a finite twist is not possible except the amplitude be zero, in which case the twist reduces to a pure translation parallel to $a$.

A wrench on a screw $a$ requires six algebraic quantities for its complete specification, and of these, five are 
required to specify the screw $a$. The sixth or metric quantity, which is called the INTENSITY OF THE WRENCH, and is denoted by $a^{\prime \prime}$, expresses the magnitude of that force which, when united with a couple, constitutes the entire wrench.

The moment of the couple is the product of the intensity of the wrench and the pitch of the screw, or in symbols, $a^{\prime \prime} p_{a}$.

If the pitch be positive (negative), the direction in which the couple acts which forms one portion of the wrench bears the same relation to the direction in which the force acts which forms the other portion of the wrench as the direction of a couple which would make a nut turn on an ordinary right-handed (left-handed) screw bears to the direction of the force parallel to the axis of the screw which would give the nut the same motion if the screw were frictionless.

If the pitch be zero, the wrench reduces to a pure force along $a$. If the pitch be infinite, then a finite wrench is not possible except the intensity be zero, in which case the wrench reduces to a couple in a plane perpendicular to $\boldsymbol{a}$.

In the case of a twisting motion about a screw $\boldsymbol{\alpha}$ the rate at which the amplitude of the twist changes may be called the TWIST VELOCITY and be denoted by $\dot{\boldsymbol{a}}^{\prime}$.

The following illustration may be useful:-

If the screw be conceived placed along the axis around which the hands of a watch turn, and if the twist be in the direction in which the hands of the watch move, then, for positive pitch the translation will be from the front of the watch to the back; for negative pitch the translation will be from the back of the watch to the front. If in this statement we interchange the words positive and negative, we have the case where the direction of the twist is opposite to the motion of the hands. 
§6. Restriction of the Forces.-It is first necessary to point out the restrictions which we shall impose upon the forces. The rigid body $M$, whose motion we are considering, is presumed to be acted upon by the same forces whenever it occupies the same position. This will necessitate that the surrounding bodies are fixed whence the forces acting on $M$ emanate. Forces such as those due to a resisting medium are excluded, because such forces do not depend on the position of the body, but on the manner in which the body is moving through that position. The same consideration excludes friction which depends on the direction in which the body is moving through the position under consideration.

But the condition that the forces shall be defined, when the position is given, is still not sufficiently precise. We might include, in this restricted group, forces which could have no existence in nature. We shall, therefore, add the condition that the system is to be one in which the contimual creation of energy is impossible.

$\S 7$. The Energy of Position. - An important consequence of this restriction is stated as follows:-The quantity of energy necessary to compel the body $M$ to move from the position $A$ to the position $B$, is independent of the toute by which the change has been effected.

Let $L$ and $M$ be two such routes, and suppose that less energy was required to make the change from $A$ to $B$ viâ $L$ than viâ $M$. Make the change viâ $L$, with the expenditure of a certain quantity of energy, and then allow the body to return viâ $M$. Now, since at every stage of the route $M$ the forces acting on the body are the same whichever way the body be moving, it follows, that in returning from $B$ to $A$ viâ $M$, the forces will give out exactly as much energy as would have been required to compel the body to move from $A$ to $B$ via $M$; but by hypothesis this exceeds the energy necessary to make 
the change viâ $L$, and hence, on the return of the body to $A$, there is a clear gain of a quantity of energy, while the position of the body and the forces are the same as at first. By successive repetitions of the process an indefinite quantity of energy could be created from nothing. This being contrary to nature, compels us to admit that the quantity of energy necessary to force the body from $A$ to $B$ is independent of the route followed.

\& 8. Theorem.-The sum of the works done in a number of twists against a wrench is equal to the work that would be done in the resultant twist.

For, by the last article, the work done in producing a given change of position is independent of the route.

\$ 9. Theorem.-We first define that by the work done in a twist against a wrench is to be understood the sum of the works done against the three forces which constitute the wrench in the movements of their points of application which are caused by the twist.

We shall assume the two lemmas-ist. The work done in the displacement of a rigid body against a force is the same at whatever point in its line of application the force acts. 2nd. The work done in the displacement of a point against a number of forces acting at that point, equals the work done in the same displacement against the resultant force.

The theorem to be proved is as follows :-The sum of the works done in a given twist against a number of wrenches, equals the work done in the same twist against. the resultant wrench.

Let $n$ wrenches, which consist of $3 n$ forces acting at $A_{1}$, \&c., $A_{3 n}$, compound into one wrench, of which the three forces act at $P, Q, R$. The force at each point $A_{k}$ may be decomposed into three forces along $P A_{k}, Q A_{k}$, $R A_{k}$. By the 2nd lemma the sum of the works $(W)$ 
done against the $3 n$ original forces, equals the sum of the works done against the $9 n$ components. It, therefore, appears from the ist lemma, that $W$ will still be the sum of the works done against the $9 n$ components, of which $3 n$ act at $P, 3 n$ at $Q, 3 n$ at $R$. Finally, by the and lemma, $W$ will also be the sum of the works done by the original twist against the three resultants formed by compounding each group at $P, Q, R$. But these resultants constitute the resultant wrench, whence the theorem has been proved.

$\S$ I0. Theorem.-From a comparison of the two last articles, we easily deduce the following theorem, which we shall find of great service throughout the essay.

If a series of twists $A_{1}, \&$ c., $A_{m}$, would compound into one twist $A$, and a series of wrenches $B_{1}, \&$ c., $B_{n}$, would compound into one wrench $B$, then the energy that would be expended or gained when the rigid body performs the twist $A$, under the influence of the wrench $B$, is equal to the algebraic sum of the $m n$ quantities of energy that would be expended or gained when the body performs severally each twist $A_{1}, \&$ c., under the influence of each wrench $B_{1}, \&$ c.

$\S$ II. Concluding Remarks.-We have now explained the conceptions, and the language in which the solution of any problem in the Dynamics of a rigid body may be presented. A complete solution of such a problem must provide us, at each epoch, with a screw, by a twist about which of an amplitude also to be specified, the body can be brought from a standard position to the position occupied at the epoch in question. It will also be of much interest to know the instantaneous screw about which the body is twisting at each epoch, as well as its twist velocity. Nor can we regard the solution as quite complete, unless we also have a clear conception of the screw on which all the forces acting on the body consti- 
tute a wrench of which we should also know the intensity.

There is one special feature which characterises that portion of the Dynamics of a rigid body which is discussed in the present essay. We shall impose no restrictions on the form of the rigid body, none on the character of the constraints by which its movements are limited, and but little on the forces to which the rigid body is submitted. The restriction which we do make is that the body, while the object of examination, remains in, or indefinitely adjacent to, its original position.

As a consequence of this restriction, we here make the remark that the amplitude of a twist is henceforth to be regarded as a small quantity.

If it be objected, that with so great a restriction as that just referred to, only a limited field of inquiry remains, the answer is as follows :-A perfectly general investigation could yield but a slender harvest of interesting or valuable results. All the problems of Physical importance are special cases of the general question. Thus, a special character in the constraints has produced the celebrated problem of the rotation of a rigid body about a fixed point. To vindicate our particular restriction it seems only necessary to remark, that the restricted inquiry still includes the theory of Equilibrium, of Impulsive Forces, and of Small Oscillations.

Whatever of novelty may be found in the following pages will, it is believed, be due to the circumstance that, with the important exception referred to, all the conditions of each problem are of absolute generality. 


\section{CHAPTER II.}

THE CYLINDROID.

12. Introduction.-We shall now ascertain the laws according to which twists (and wrenches) must be compounded together, that is to say, we shall determine the single screw, one twist (or wrench) about which will produce the same effect on the body as two or more given twists (or wrenches) about two or more given screws. It will be found to be a fundamental point of the present theory that the rules for the composition of twists and of wrenches are identical.*

$\S$ 13. On the Virtual Coefficient of a Pair of Screws.Suppose a rigid body be acted upon by a wrench on a screw $\beta$, of which the intensity is $\beta^{\prime \prime}$. Let the body receive a twist of small amplitude $\boldsymbol{a}^{\prime}$ around a screw $a$. It is proposed to find an expression either for the energy required to effect the displacement, or the work done if the displacement be permitted.

Let $d$ be the shortest distance between $a$ and $\beta$, and let $O$ be the anglet between $\boldsymbol{\alpha}$ and $\beta$. Take $\boldsymbol{a}$ as the axis of $x$, the common perpendicular to $a$ and $\beta$ as the axis

* That the source of the analogy between the composition of forces and of rotations lies in the general principles of virtual velocities, has been proved by Rodrigues (Liouville's Journal, t. 5, I840, p. 436).

+ Perhaps the best convention to distinguish between $O$ and its supplement is the following :-Suppose the common perpendicular to be an ordinary righthanded screw, and that there is a nut on this screw to which $a$ is attached. If, then, the nut be turned so as to make a approach $\beta$ (that is, to make the length of the common perpendicular diminish), the angle less than $\pi$ through which $a$ has turned when it has become parallel to $\beta$ is the angle $O$. 
of $y$, and a line perpendicular to $x$ and $y$ for $z$. If we resolve the wrench on $\beta$ into forces $X, Y, Z$, parallel to the axes, and couples of moments $L, M, N$, in planes perpendicular to the axes we shall have

$$
X=\beta^{\prime \prime} \cos O ; Y=\beta^{\prime \prime} \sin O ; Z=0
$$

$L=\beta^{\prime \prime} p_{\beta} \cos O-\beta^{\prime \prime} d \sin O ; M=\beta^{\prime \prime} p_{\beta} \sin O+\beta^{\prime \prime} d \cos O$;

$$
N=0 \text {. }
$$

We thus reduce the given wrench to four wrenches, viz., two forces 'and two couples, and we reduce the given twist to two twists, viz., one rotation and one translation. By the principle of $\S$ Io the work done by the given twist against the given wrench must equal the sum of the eight quantities of work done by each of the two component twists against each of the four component wrenches. Six of these quantities are evanescent. In fact a rotation through the angle $\boldsymbol{a}^{\prime}$ around the axis of $x$ can only do work against $L$, the amount being

$$
\boldsymbol{\alpha}^{\prime} \beta^{\prime \prime}\left(p_{\beta} \cos O-d \sin O\right) \text {. }
$$

The translation $p_{a} a^{\prime}$ parallel to the axis of $x$ can only do work against $X$, the amount being

$$
a^{\prime} \beta^{\prime \prime} p_{a} \cos O \text {. }
$$

Thus, the total quantity of work done is

$$
\boldsymbol{a}^{\prime} \beta^{\prime \prime}\left\{\left(p_{a}+p_{\beta}\right) \cos O-d \sin O\right\} \text {. }
$$

The expression

$$
\left(p_{a}+p_{\beta}\right) \cos O-d \sin O,
$$

is of great importance in the present theory.* It is

* The theory of screws has many points of connexion with recent geometrical speculations on the linear complex, by the late Dr. Plücker and Dr. Felix Klein. Thus the latter has shown, (Mathematische Annalen, Band II., p. 368), 
called the virtual coefficient of the two screws $a$ and $\beta$, and may be denoted by the symbol

$$
2 \varpi_{a \beta} \text {. }
$$

\$ I4. Symmetry of the Virtual Coefficient.-One property of the virtual coefficient is of the utmost importance. If the two screws $\alpha$ and $\beta$ be interchanged, the virtual coefficient remains unaltered. The identity of the laws of composition of twists and wrenches can be deduced from this property, ${ }^{*}$ and also the Theory of Reciprocal Screws.

\$15. Composition of Twists and Wrenches.-Suppose three twists about three screws $a, \beta, \gamma$, possess the property that the body after the last twist has the same position which it had before the first: then the amplitudes of the twists, as well as the geometrical relations of the screws, must satisfy certain conditions. The particular nature of these conditions does not concern us at present, although it will be fully developed hereafter.

We may at all events conceive the following method of ascertaining these conditions:-

It follows from $\S 10$, that the sum of the works done in the twists about $a, \beta, \gamma$, against a wrench, on any screw $\eta$, must be zero, whence

$$
a^{\prime} \varpi_{a \eta}+\beta^{\prime} \varpi_{\beta \eta}+\gamma^{\prime} \varpi_{\gamma \eta}=0 .
$$

This equation is a type of an indefinite number (of which six are independent) which may be obtained by

that if $p_{a}$ and $p_{\beta}$ be each the "hauptparameter" of a linear complex, and if

$$
\left(p_{\alpha}+p_{\beta}\right) \cos O-d \sin O=0,
$$

where $d$ and $O$ relate to the principal axes of the complexes, that then the two complexes possess a special relation and are said to be in "involution."

- This remark, or what is equivalent thereto, is due to Dr. Felix Klein (Math. Ann., vol. iv., p. 413). 
choosing different screws for $\eta$. From each group of three equations the amplitudes can be eliminated, and four of the equations thus obtained will involve all the purely geometrical conditions as to direction, situation, and pitch, which must be fulfilled by the screws when three twists can neutralize each other.

But now suppose that three wrenches equilibrate on the three screws $a, \beta, \gamma$. Then ( $\$ 10$ ) the sum of the works done in a twist about any screw $\eta$ against the three wrenches must be zero, whence

$$
a^{\prime \prime} \varpi_{a \eta}+\beta^{\prime \prime} \varpi_{\beta \eta}+\gamma^{\prime \prime} \varpi_{\gamma \eta}=0,
$$

and an indefinite number of similar equations must be satisfied.

By comparing this system of equations with that previously obtained, it is obvious that the geometrical conditions imposed on the screws $\alpha, \beta, \gamma$, in the two cases are identical, and that the amplitudes of the three twists which neutralise are, respectively, proportional to the intensities of the three wrenches which equilibrate.

When three twists (or wrenches) neutralise, then a twist (or wrench) equal and opposite to one of them must be the resultant of the other two, and hence it follows that the laws for the composition of twists and of wrenches must be identical.

$\S$ I6. The Cylindroid.-We now proceed to study the composition of twists and wrenches, and we select twists for this purpose, though wrenches would have been equally convenient.

A body receives twists about three screws; under what conditions will the body, after the last twist, occupy the same position which it had before the first.

The problem may also be stated thus :-It is required to ascertain the single screw, a twist about which would produce the same effect as any two given twists. We 
shall first examine a special case, and from it we shall deduce the general solution.

Take, as axes of $x$ and $y$, two screws $\alpha, \beta$, intersecting at right angles, whose pitches are $p_{s}$ and $p_{\beta}$. Let a body receive twists about these screws of amplitudes $\theta^{\prime} \cos l$ and $\theta^{\prime} \sin l$. The translations parallel to the axes of $x$ and $y$ will then be $p_{a} \theta^{\prime} \cos l$ and $p_{\beta} \theta^{\prime} \sin l$. The resultant of the two translations may be resolved into two components, of which $\theta^{\prime}\left(p_{a} \cos ^{2} l+p_{\beta} \sin ^{2} l\right)$ is parallel to the direction of that axis, a rotation about which is equivalent to the two given rotations, while $\theta^{\prime} \sin l \cos l\left(p_{a}-p_{\beta}\right)$ is perpendicular to the same line. The latter component has the effect of transferring the resultant axis of the rotations to a distance $\sin l \cos l\left(p_{a}-p_{\beta}\right)$, the axis moving parallel to itself in a plane perpendicular to that which contains $\boldsymbol{a}$ and $\beta$. The two original twists about $\boldsymbol{\alpha}$ and $\beta$ are therefore compounded into a single twist of amplitude $\theta^{\prime}$ about a screw $\theta$ whose pitch is

$$
p_{a} \cos ^{2} l+p_{\beta} \sin ^{2} l \text {. }
$$

The position of the screw $\theta$ is defined by the equations

$$
\begin{aligned}
y & =x \tan l, \\
z & =\left(p_{a}-p_{\beta}\right) \sin l \cos l .
\end{aligned}
$$

Eliminating $l$ we have the equation

$$
z\left(x^{2}+y^{2}\right)-\left(p_{a}-p_{\beta}\right) x y=0 .
$$

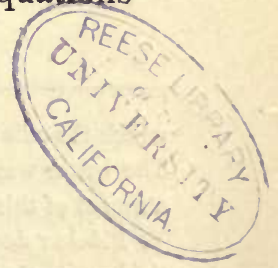

The conoidal cubic surface represented by this equation has been called the cylindroid.*

- This surface has been described by Plücker (Neue Geometrie des Raumes, p. 97); he arrives at it as follows :-Let $\Omega=0$, and $\Omega^{\prime}=0$ represent two linear complexes of the first degree, then all the complexes formed by giving $\mu$ different values in the expression $\Omega+\mu \Omega^{\prime}=0$ form a system of which the axes lie on the surface $z\left(x^{2}+y^{2}\right)-\left(k^{0}-k_{0}\right) x y=0$. The parameter of any complex of 
Each generating line of the surface is conceived to be the residence of a screw, the pitch of which is determined by the expression $p_{a} \cos ^{2} l+p_{\beta} \sin ^{2} l$.

We shall now show that a cylindroid can be described so as to contain any two screws. When a cylindroid is said to contain a screw, it is not only meant that the screw is one of the generators of the surface, but that the pitch of the screw is identical with the pitch appropriate to the generator with which the screw coincides.

Let the two given screws be $\theta$ and $\phi$, the length of their common perpendicular be $h$, and the angle between the two screws be $A$; we shall show that by a proper choice of the origin, the axes, and the constarits $p_{a}$ and $p_{\beta}$, a cylindroid can be found which contains $\theta$ and $\phi$.

If $l, m$ be the angles which two screws on a cylindroid make with the axis of $x$, and if $z_{1}, z_{2}$ be the corresponding values of $z$, we have the equations

$$
\begin{array}{cl}
p_{\theta}=p_{a} \cos ^{2} l+p_{\beta} \sin ^{2} l, & z_{1}=\left(p_{a}-p_{\beta}\right) \sin l \cos l, \\
p_{\phi}=p_{\alpha} \cos ^{2} m+p_{\beta} \sin ^{2} m . & z_{2}=\left(p_{a}-p_{\beta}\right) \sin m \cos m . \\
A=l-m, & h=z_{1}-z_{2},
\end{array}
$$

which the axis makes an angle $\omega$ with the axis of $x$ is $k=k^{0} \cos ^{2} \omega+k_{0} \sin ^{2} \omega$. The writer was informed by Dr. Felix Klein that Plücker had also constructed a model of this surface.

Plücker does not appear to have contemplated the mechanical and kinematical properties of the cylindroid, with which alone we are concerned; but it is worthy of remark that the distribution of pitch which is presented by physical considerations is exactly the same as the distribution of parameter upon the generators of the surface, which was fully discussed by Plücker in connexion with his theory of the linear complex.

The name cylindroid was suggested by Professor Cayley in reply to a request of the writer. The word originated in the following construction for the surface, which was also communicated by Professor Cayley. Cut the cylinder $x^{2}+y^{2}=\left(p_{\beta}-p_{\alpha}\right)^{2}$ in an ellipse by the plane $z=x$, and consider the line $x=0, y=p_{\beta}-p_{\alpha}$. If any plane $z=c$ cuts the ellipse in the points $A, B$ and the line in $C$, then $C A, C B$ are two generating lines of the surface. 


$$
\begin{gathered}
p_{\alpha}-p_{\beta}=\frac{\sqrt{h^{2}+\left(p_{\theta}-p_{\phi}\right)^{2}}}{\sin A} \\
p_{a}+p_{\beta}=p_{\theta}+p_{\phi}-h \cot A . \\
l=\frac{1}{2}\left(A+\tan ^{-1} \frac{p_{\theta}-p_{\phi}}{h}\right) \cdot \quad z_{1}=\frac{1}{2}\left(p_{\beta}-p_{a}\right) \cot A+\frac{h}{2},
\end{gathered}
$$

with similar values for $m$ and $z_{2}$. It is therefore obvious that the cylindroid is determined, and that the solution is unique.

It will often be convenient to denote by $(\theta, \phi)$ the cylindroid drawn through the two screws $\theta$ and $\phi$.

$\S$ I7. General Property. - The general property of the cylindroid, which is of importance for our present purpose, may be thus stated. If a body receive twists about three screws on a cylindroid, and if the amplitude of each twist be proportional to the sine of the angle between the two non-corresponding screws, then the body after the last twist will occupy the same position which it did before the first.

The proof of this theorem must, according to $\S \mathrm{I}_{5}$; involve the proof of the following:-If a body be acted upon by wrenches about three screws on a cylindroid, and if the intensity of each wrench be proportional to the sine of the angle between the two non-corresponding screws, then the three wrenches equilibrate.

The former of these properties of the cylindroid is thus proved:-Take any three screws $\theta, \phi, \psi$, upon the surface which make angles $l, m, n$, with the axis of $x$, and let the body receive twists about these screws of amplitudes $\theta^{\prime}, \phi^{\prime}, \psi^{\prime}$. Each of these twists can be decomposed into two twists about the screws $\boldsymbol{\alpha}$ and $\boldsymbol{\beta}$ which lie along the axes of $x$ and $y$. The entire effect of the three twists is, therefore, reduced to two rotations around the axes of $x$ and $y$, and two translations parallel to these axes. 
The rotations are through angles equal respectively to

and

$$
\theta^{\prime} \cos l+\phi^{\prime} \cos m+\psi^{\prime} \cos n
$$

$$
\theta^{\prime} \sin l+\phi^{\prime} \sin m+\psi^{\prime} \sin n \text {. }
$$

The translations are through distances equal to

$$
p_{a}\left(\theta^{\prime} \cos l+\phi^{\prime} \cos m+\psi^{\prime} \cos n\right)
$$

and

$$
p_{\beta}\left(\theta^{\prime} \sin l+\phi^{\prime} \sin m+\psi^{\prime} \sin n\right) \text {. }
$$

These four quantities vanish if

$$
\frac{\theta^{\prime}}{\sin (m-n)}=\frac{\phi^{\prime}}{\sin (n-l)}=\frac{\psi^{\prime}}{\sin (l-m)},
$$

and hence the fundamental property of the cylindroid has been proved.

The cylindroid affords the means of compounding two twists (or two wrenches) by a rule as simple as that which the parallelogram of force provides for the composition of two intersecting forces. Draw the cylindroid which contains the two screws; select the screw on the cylindroid which makes angles with the given screws whose sines are in the inverse ratio of the amplitudes of the twists (or the intensities of the wrenches); a twist (or wrench) about the screw so determined is the required resultant. The amplitude of the resultant twist (or the intensity of the resultant wrench) is proportional to the diagonal of a parallelogram of which the two sides are parallel to the given screws, and of lengths proportional to the given amplitudes (or intensities).

$\S$ I8. Particular Cases.-If $p_{a}=p_{\beta}$ the cylindroid reduces to a plane, and the pitches of all the screws are equal. If the pitches be all zero, then the general property of the cylindroid reduces to the well known construction for the resultant of two intersecting forces, or of rotations about two intersecting axes. If the pitches 
be all infinite, the general property reduces to the construction for the composition of two translations or of two couples.

$\S$ I9. Form of the Cylindroid.-The equation of the surface only contains the single parameter $p_{a}-p_{\beta}$, consequently all cylindroids are similar surfaces only differing in absolute magnitude.

The curved portion of the surface is contained between the two parallel planes $z= \pm\left(p_{a}-p_{\beta}\right)$, but it is to be observed that the nodal line $x=0, y=0$, also lies upon the surface.

The intersection of the nodal line with a plane is a double point (connode) or a conjugate point (acnode) upon the curve in which the plane is cut by the cylindroid according as the point does lie or does not lie between the two bounding planes.

A model of a portion of the cylindroid is represented in the frontispiece. In order to realize from the model the actual form of the surface, the diameter of the central cylinder must be conceived to be evanescent, and the radiating wires must be extended to infinity.

$\S 20$. The Pitch Conic.-Besides being acquainted with the form of the cylindroid, it is also very useful to have a clear view of the distribution of pitch upon the screws contained on the surface. The surface being given, one arbitrary element must be further specified before that distribution is known. If, however, two screws be given, then both the surface and the distribution are determined. Any constant quantity may be added to all the pitches of a certain distribution, and the distribution thus modified is still a possible one.

Let $p_{\theta}$ be the pitch of a screw $\theta$ on the cylindroid which makes an angle $l$ with the axis of $x$; then ( $\S I I)$

$$
p_{\theta}=p_{a} \cos ^{2} l+p_{\beta} \sin ^{2} l \text {. }
$$


Draw in the plane $x, y$, the conic

$$
p_{a} x^{2}+p_{\beta} y^{2}=H \text {, }
$$

where $H$ is any constant; and if $r$ be the radius vector which makes an angle $l$ with the axis of $x$, we have

$$
p_{\theta}=\frac{H}{r^{2}}
$$

whence the pitch of each screw on a cylindroid is proportional to the inverse square of the parallel diameter of the pitch conic.

Being given the cylindroid, we require further to know the eccentricity of the pitch conic, and then the pitches of all the screws are determined.

$\S 2$ I. Summary.-It is one of the main objects of the present essay to associate a geometrical conception with the solution of each problem. To do this effectively we shall often have occasion to make use of the principle demonstrated in this chapter, viz.,

That a cylindroid can be drawn so that not only shall two of its generators coincide with any two given screws a and $\beta$, but that when all the generators of the surface become screws by having pitches assigned to them according to the law of distribution enunciated in $\$ 20$, the pitches assigned to the generators which coincide with $a$ and $\beta$ shall be equal to the given pitches of $a$ and $\beta$. 


\section{CHAPTER III.}

RECIPROCAL SCREWS.

22. Reciprocal Screws.-If a body only free to twist about a screw $\boldsymbol{a}$ be in equilibrium, though acted upon by a wrench on the screw $\beta$, then conversely a body only free to twist about the screw $\beta$ will be in equilibrium, though acted upon by a wrench on the screw $\boldsymbol{\alpha}$.

The principle of virtual velocities states, that if the body be in equilibrium the work done in a small displacement against the external forces must be zero; but the condition for this is, that the virtual coefficient should vanish $(\S \mathrm{I})$, or

$$
\left(p_{a}+p_{\beta}\right) \cos O-d \sin O=0 .
$$

The symmetry shows that precisely the same condition is required whether the body be free to twist about $a$, while the wrench act on $\beta$, or vice versâ. $A$ pair of screws are said to be reciprocal when their virtual coefficient is zero.

§23. Particular Instances:-Parallel or intersecting screws are reciprocal when the sum of their pitches is zero. Screws at right angles are reciprocal either when they intersect, or when one of the pitches is infinite. Two screws of infinite pitch are reciprocal, because a couple could not move a body which was only susceptible of translation. A screw whose pitch is zero or infinite is reciprocal to itself.*

* For other particular instances see Professor Everett "On a New Method in Statics and Kinematics," \$27; “Messenger of Mathematics," New Series, No. 39,1874 . 
$\S 24$. Screw Reciprocal to Cylindroid.-If a screw $\eta$ be reciprocal to two given screws $\theta$ and $\phi$, then $\eta$ is reciprocal to every screw on the cylindroid $(\theta, \phi)$.

For a body only free to twist about $\eta$ would be undisturbed by wrenches on $\theta$ and $\phi$; but a wrench on any screw $\psi$ of the cylindroid can be resolved into wrenches on $\theta$ and $\phi$; therefore a wrench on $\psi$ cannot disturb a body only free to twist about $\eta$; therefore $\psi$ and $\eta$ are reciprocal. We may say for brevity that $\eta$ is reciprocal to the cylindroid.

$\eta$ cuts the cylindroid in three points, ${ }^{*}$ and one screw of the cylindroid passes through each of these three points ; these three screws must, of course, be reciprocal to $\eta$. Now two intersecting screws can only be reciprocal when they are at right angles, or when the sum of their pitches is zero. The pitch of the screw upon the cylindroid which makes an angle $l$ with the axis of $x$ is

$$
p_{a} \cos ^{2} l+p_{\beta} \sin ^{2} l \text {. }
$$

This is also the pitch of the screw $\pi-l$. There are, therefore, two screws of any given pitch; but there cannot be more than two. It follows that $\eta$ can at most intersect two screws upon the cylindroid of pitch equal and opposite to its own; and, therefore, $\boldsymbol{\eta}$ must be perpendicular to the third screw. $\dagger$ Hence any screw reciprocal to a cylindroid must intersect one of the generators at right angles. We easily infer, also, that a line intersecting one screw of a cylindroid at right angles, must cut the surface again in two points, the screws passing through which have equal pitch.

$\S 25$. Reciprocal Cone.-From any point $P$ perpen-

* Every right line meets a surface of the third degree in three points. Salmon, "Analytic Geometry of Three Dimensions," 2nd Ed., p. I4.

+ The writer may, perhaps, be excused for adding that it was the perception of this point which first gave him clear views on the subject of the present volume. 
diculars can be let fall upon the generators of the cylindroid, and if to these perpendiculars pitches are assigned which are equal in magnitude and opposite in sign to the pitches of the two remaining screws on the cylindroid intersected by the perpendicular, then the perpendiculars form a cone of reciprocal screws.

We shall now prove that this cone is of the second order, and we shall show how it can be constructed.

Let $O$ be the point from which the cone is to be drawn, and through $O$ let a line $O T$ be drawn which is parallel to the nodal line, and, therefore, perpendicular to all the generators. This line will cut the cylindroid in one real point $T$ (Fig. 2), the two other points of intersection coalescing into the infinitely distant point in which $O T$ intersects the nodal line.

Draw a plane through $T$ and through the screw $L M$ which, lying on the cylindroid, has the same pitch as the screw through $T$. Now this plane must cut the cylindroid in a conic section, for the line $L M$ and the conic will then make up the curve of the third degree, in which the plane must cut the surface.* Also since the entire cylindroid (or at least its curved portion) is included between two parallel planes, $\S 19$, it follows, that this conic must be an ellipse.

We shall now prove that

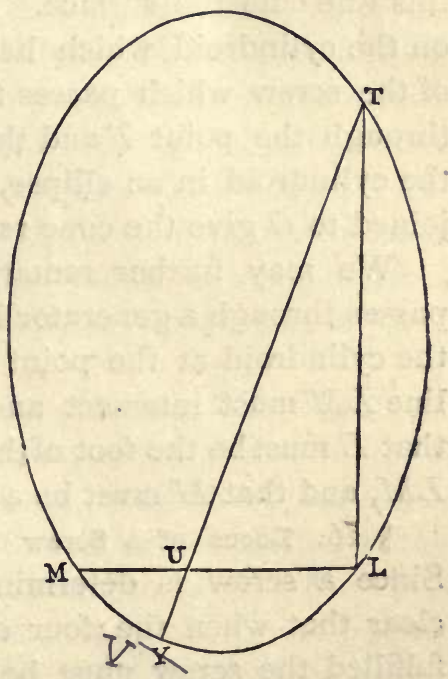

Fig. 2.

* Salmon, "Analytic Geometry of Three Dimensions," 2nd Ed., p. I4. 
this ellipse is the locus of the feet of the perpendiculars let fall from $O$ on the generators of the cylindroid. Draw in the plane of the ellipse any line $T U V$ through $T$; then, since this line intersects two screws of equal pitch in $T$ and $U$, it must be perpendicular to that generator of the cylindroid which it meets at $V$. This generator is, therefore, perpendicular to the plane of $O T$ and $V T$, and, therefore, to the line $O V$. It follows that $V$ must be the foot of the perpendicular from $O$ on the generator through $V$, and that, therefore, the cone drawn from $O$ to the ellipse $T L V M$ is the cone required.

We hence deduce the following construction for the cone of reciprocal screws which can be drawn to a cylindroid from any point $O$.

Draw through $O$ a line parallel to the nodal line of the cylindroid, and let $T$ be the one real point in which this line cuts the surface. Find the second screw $L M$ on the cylindroid which has a pitch equal to the pitch of the screw which passes through $T$. A plane drawn through the point $T$ and the straight line $L M$ will cut the cylindroid in an ellipse, the various points of which joined to $O$ give the cone required.

We may further remark that as the plane TLM passes through a generator it must be a tangent plane to the cylindroid at the point $L$, while at the point $M$ the line $L M$ must intersect another generator.* It follows that $L$ must be the foot of the perpendicular from $T$ upon $L M$, and that $M$ must be a point upon the nodal line.

$\S 26$. Locus of a Screw Reciprocal to Four Screws.Since a screw is determined by five quantities, it is clear that when the four conditions of reciprocity are fulfilled the screw must be confined to a certain ruled surface. Now this surface can be no other than a cylin-

* Salmon, "Analytic Geometry of Three Dimensions," 2nd Ed., p. 348. 
droid. For, suppose that three screws $\lambda, \mu, \nu$, which were reciprocal to the four given screws did not lie on the same cylindroid, then any screw $\phi$ on the cylindroid $(\lambda, \mu)$, and any screw $\psi$ on the cylindroid $(\lambda, v)$ must also fulfil the conditions, and so must also every screw on the cylindroid $(\phi, \psi)(\S 4)$. We should thus have the screws reciprocal to four given screws, limited not to one surface, but to a family of surfaces, which is impossible. The construction of the cylindroid which is the locus of all the screws reciprocal to four given screws, may be effected in the following manner :-

Let $a, \beta, \gamma, \delta$ be the four screws, of which the pitches are in descending order of magnitude. Draw the cylindroids $(a, \gamma)$ and $(\beta, \delta)$. If $\sigma$ be a linear magnitude intermediate between $p_{\beta}$ and $p_{\gamma}$, it will be possible to choose two screws of pitch $\sigma$ on $(a, \gamma)$, and also two screws of pitch $\sigma$ on $(\beta, \delta)$. Draw the two transversals which intersect the four screws thus selected;* attribute to each of these transversals the pitch $-\sigma$, and denote the screws thus produced by $\theta, \phi$. Since intersecting screws are reciprocal when the sum of their pitches is zero, it follows that $\theta$ and $\phi$ must be reciprocal to the cylindroids $(a, \gamma)$ and $(\beta, \delta)$. Hence all the screws on the cylindroid $(\theta, \phi)$ must be reciprocal to $a, \beta, \gamma, \delta$, and thus the problem has been solved.

\$27. Screw Reciprocal to Five Screws.-The problem of the determination of a screw reciprocal to five given screws must admit of a finite number of solutions, because the number of conditions to be fulfilled is the same as the number of disposable constants. Now it is very important to observe that that number must be one. For if two screws could be found which fulfilled the neces-

* Two lines can be drawn which will intersect four non-intersecting lines. Salmon, "Analytic Geometry of Three Dimensions," 2nd Ed., page 426. 
sary conditions, then these conditions would be equally fulfilled by every screw on the cylindroid determined by those screws ( $\$ 24)$, and therefore the number of solutions. of the problem would not be finite.

The construction of the screw whose existence is thus demonstrated, can be effected by the results of the last article. Take any four of the five screws, and draw the reciprocal cylindroid which must contain the required screw. Any other set of four will give a different cylindroid, which also contains the required screw. Thesecylindroids must therefore intersect in the single screw, which is reciprocal to the five given screws.

$\$ 28$. Screw upon a Cylindroid Reciprocal to a Given. Screw.-Let $\varepsilon$ be the given screw, and let $\lambda, \mu, v, \rho$ be any four screws reciprocal to the cylindroid; then the single screw $\eta$, which is reciprocal to the five screws $\varepsilon, \lambda, \mu, v, \rho$, must lie on the cylindroid because it is reciprocal to $\lambda, \mu, \nu, \rho$, and therefore $\eta$ is the screw required.

The solution must be unique, for if a second screw were reciprocal to $\varepsilon$, then the whole cylindroid would be reciprocal to $\varepsilon$; but this is not the case unless $\varepsilon$ fulfil certain conditions ( $\$ 24)$.

$\S 29$. Properties of the Cylindroid.-We add here a few properties of the cylindroid for which the writer is principally indebted to his friend Dr. Casey.

The ellipse in which a tangent plane cuts the cylindroid has a circle for its projection on a plane perpendicular to the nodal line, and the radius of the circle is the minor axis of the ellipse.

The difference of the squares of the axes of the ellipse: is constant wherever the tangent plane be situated.

The minor axes of all the ellipses lie in the same plane.

The line joining the points in which the ellipse is cut by two screws of equal pitch on the cylindroid is parallel to the major axis. 
The line joining, the points in which the ellipse is cut by two intersecting screws on the cylindroid, is parallel to the minor axis.

All the screws which lie in a plane and are reciprocal to a cylindroid envelope a conic. The relation of this conic to the cubic in which the plane cuts the cylindroid might have some geometrical interest.

As we have no physical applications to make of these theorems, the demonstrations are not given. 


\section{$(28)$}

\section{CHAPTER IV.}

\section{SCREW CO-ORDINATES.}

\$30. Introduction.-We are accustomed, in ordinary statics, to resolve the forces acting on a rigid body into three forces acting along given directions at a point and three couples in three given planes. In the present theory we are, however, led to regard a force as a wrench on a screw, of which the pitch is zero, and a couple as a wrench on a screw of which the pitch is infinite. The familiar process just referred to is, therefore, only a special case of the more general method of resolution by which the intensities of the six wrenches on six given screws can be determined, so that, when these wrenches are compounded together, they shall constitute a wrench of given intensity on a given screw.*

The problem which has to be solved may be stated in a more symmetrical manner as follows :-

To determine the intensities of the seven wrenches on seven given screws, such that, when these wrenches are applied to a rigid body, which is entirely free to move in any way, they shall equilibrate.

The solution of this problem is identical ( $\$ 15)$ with that which may be enunciated as follows :-

To determine the amplitudes of seven small twists about seven given screws, such that, if these twists be

* If all the pitches be zero, the problem stated above reduces to the determination of the six forces along six given lines which shall be equivalent to a given force. If further, the six lines of reference form the edges of a tetrahedron, we have a problem which has been solved by Möbius, Crelle's Journal, t. xviii., p. $20 \%$. 
applied to a rigid body in succession, the body after the last twist shall occupy the same position which it had before the first.

The problem we have last stated has been limited as usual to the case where the amplitudes of the twists are small quantities, so that the motion of each particle produced by each twist is sensibly rectilinear. Were it not for this condition a distinct solution would be required for every variation of the order in which the successive twists were imparted.

If the number of screws were greater than seven, then both problems would be indeterminate; if the number were less than seven, then both problems would be impossible (unless the screws were specially related); the number of screws being seven, the problem of the determination of the ratios of the seven intensities (or amplitudes) has, in general, one solution. We shall solve this for the case of wrenches.

Let the seven screws be $a, \beta, \gamma, \delta, \varepsilon, \zeta, \eta$. Find the screw $\psi$ which is reciprocal to $\gamma, \delta, \varepsilon, \zeta, \eta$. Let the seven wrenches act upon a body only free to twist about $\psi$. The reaction of the constraints which limit the motion of the body will neutralize every wrench on a screw reciprocal to $\psi(\$ 22)$. We may, therefore, so far as a body thus circumstanced is concerned, discard all the wrenches except those on $\boldsymbol{\alpha}$ and $\beta$. Draw the cylindroid $(\alpha, \beta)$, and determine thereon the screw $\rho$ which is reciprocal to $\psi$. The body will not be in equilibrium unless the wrenches about $\boldsymbol{\alpha}$ and $\beta$ constitute a wrench on $\rho$, and hence the ratio of the intensities $\alpha^{\prime \prime}$ and $\beta^{\prime \prime}$ is determined. By a similar process the ratio of the intensities of the wrenches on any other pair of the seven screws may be determined, and thus the problem has been solved.

$\S 3$ I. Intensities of the Components.-Let the six screws of reference be $\omega_{1}, \&$ c. $\omega_{6}$, and let $\rho$ be a given screw 
on which is a wrench of given intensity $\rho^{\prime \prime}$. Let the intensities of the components be $\rho_{1}{ }^{\prime \prime}, \&$ c. $\rho_{6}{ }^{\prime \prime}$, and let $\eta$ be any screw. By the principle of $\S$ 1o, a twist about $\eta$ must do the same quantity of work acting directly against the wrench on $\rho$ as the sum of the six quantities of work which would be done by the same twist against each of the six components of the wrench on $\rho$. We, therefore, have the equation (using the notation of $p$. r3)

$$
\rho^{\prime \prime} \varpi_{\eta \rho}=\rho_{1}^{\prime \prime} \varpi_{n \omega_{1}}+\& \mathrm{c} .+\rho_{6}^{\prime \prime} \varpi_{n \omega_{6}} \text {. }
$$

By taking five other screws in place of $\eta$, five more equations are obtained, and from the six equations thus found $\rho_{1}{ }^{\prime \prime}, \&$ c. $\rho_{6}{ }^{\prime \prime}$ can be determined. This process will be greatly simplified by judicious choice of the six screws of which $\eta$ is the type. Let $\eta$ be reciprocal to $\omega_{2}$, \&c. $\omega_{6}$, then $\sigma_{n \omega_{2}}=0 \&$ c. $\varpi_{\eta \omega_{6}}=0$, and we have

$$
\rho^{\prime \prime} \varpi_{\eta \rho}=\rho_{1}^{\prime \prime} \varpi_{n \omega_{1}} \text {. }
$$

From this equation $\rho_{1}{ }^{\prime \prime}$ is at once determined, and by five similar equations the intensities of the five remaining components may be likewise found.

Precisely similar is the investigation which determines the amplitudes of the six twists about the six screws of reference into which any given twist may be decomposed.

$\S 32$. The Intensity of the Resultant may be expressed in terms of the intensities of its components on the six screws of reference.

Let $\rho$ be any screw of pitch $p_{\rho}$, and let $p_{1}, \varepsilon^{\circ} c . p_{6}$ be the pitches of the six screws of reference $\omega_{1}$, \&c. $\omega_{6}$; then taking for $\boldsymbol{\eta}$ in $(\$ 26)$, each of the screws of reference in succession, and remembering that the virtual coefficient of two coincident screws is simply double the pitch, we have the following equations:-

$$
\begin{aligned}
& \rho^{\prime \prime} \tau_{p \omega_{1}}=\rho_{1}^{\prime \prime} p_{1}+\rho_{2}^{\prime \prime} \pi_{\omega_{1} \omega_{3}}+\& c .+\rho_{6}^{\prime \prime} \varpi_{\omega_{1} \omega_{6}} \\
& \text { \&c. }=\& \mathrm{c} \text {. } \\
& \rho^{\prime \prime} \pi_{\rho \omega_{6}}=f_{1}{ }^{\prime \prime} \varpi_{\omega 6} \omega_{1}+\& c_{0}+\rho_{5}{ }^{\prime \prime} \varpi_{\omega_{6} \omega_{s}}+\rho_{6}{ }^{\prime \prime} p_{6} .
\end{aligned}
$$


But taking the screw $\rho$ in place of $\eta$ we have

$$
\rho^{\prime \prime} p_{p}=\rho_{1}{ }^{\prime \prime} \varpi_{\rho \omega_{1}}+\& c_{0}+\rho_{6}^{\prime \prime} \pi_{\rho \omega_{0}} \text {. }
$$

Substituting for $\varpi_{\rho \omega_{1}} \&$ c. $\varpi_{\rho \omega_{\varepsilon}}$ from the former equations, we deduce

$$
p_{\rho} \rho^{\prime \prime 2}=\Sigma\left(p_{1} \rho_{1}{ }^{\prime \prime 2}\right)+2 \Sigma\left(\rho_{1}{ }^{\prime \prime} \rho_{2}{ }^{\prime \prime} \varpi_{\omega_{1} \omega_{2}}\right) .
$$

This result may recall the well-known expression for the square of a force acting at a point in terms of its components along three axes passing through the point. This expression is greatly simplified when the three axes are rectangular, and we shall now show that by a special disposition of the screws of reference, a corresponding simplification can be made in the formula just written.

$\$ 33$. Co-Reciprocal Screws. -We have hitherto chosen the six screws of reference quite arbitrarily; we now proceed in a different manner. Take for $\omega_{1}$, any screw ; for $\omega_{2}$, any screw reciprocal to $\omega_{1}$; for $\omega_{3}$, any screw reciprocal to $\omega_{1}$ and $\omega_{2}$; for $\omega_{4}$, any screw reciprocal to $\omega_{1}$, $\omega_{2}, \omega_{3}$; for $\omega_{5}$, any screw reciprocal to $\omega_{1}, \omega_{2}, \omega_{3}, \omega_{4}$; for $\omega_{6}$, the screw reciprocal to $\omega_{1}, \omega_{2}, \omega_{3}, \omega_{4}, \omega_{5}$.

A group constructed in this way possesses the property that each pair of screws is reciprocal. Any set of screws not exceeding six, of which each pair is reciprocal, may be called for brevity a set of co-reciprocals.*

Thirty constants determine a group of six screws. If the group be co-reciprocal, fifteen conditions must be fulfilled; we have, therefore, fifteen elements still disposable, so that we are always enabled to select a coreciprocal group with special appropriateness to the problem under consideration.

* Dr. Klein has discussed (Math. Ann. Band II. p. 204), six linear complexes, of which each pair are in involution. If the axes of these complexes be regarded as screws, of which the " auptparameters" are the pitches, then these six screws will be co-reciprocal. 
The facilities presented by rectangular axes for questions connected with the dynamics of a particle have perhaps their analogues in the conveniences which arise from the use of co-reciprocal groups of screws in the present theory.

If the six screws of reference be co-reciprocal, then the formula of the last section assumes the very simple form

$$
p_{p} \rho^{\prime \prime 2}=p_{1} \rho_{1}^{1 / 2}+\& c .+p_{6} \rho_{6}{ }^{1 / 2}
$$

§34. Co-ordinates of a Wrench.-We shall henceforth usually suppose that the screws of reference are co-reciprocal. We may also speak of the co-ordinates of a wrench,* meaning thereby the intensities of its six components on the six screws of reference. So also we may speak of the co-ordinates of a twist, meaning thereby the amplitudes of its six components about the six screws of reference.

The co-ordinates of a wrench of intensity $\rho^{\prime \prime}$ on the screw $\rho$ are denoted by $\rho_{1}^{\prime \prime}, \&$ c. $\rho_{6}{ }^{\prime \prime}$. The co-ordinates of a twist of amplitude $\rho^{\prime}$ about $\rho$ are denoted by $\rho_{1}^{\prime}, \&$ c. $\rho_{6}^{\prime}$.

$\S 35$. The Work done in a twist of amplitude $\boldsymbol{a}^{\prime}$ about a screw $\alpha$, against a wrench of intensity $\beta^{\prime \prime}$ on the screw $\beta$, can be expressed in terms of the co-ordinates.

Replace the twist and the wrench by their respective components about the co-reciprocals. Then the total work done will be equal to the sum of the thirty-six quantities of work done by each component twist against each component wrench $(\S$ IO). Since the screws are co-

* Plücker has introduced the conception of the six co-ordinates of a system of forces-Phil. Trans., vol. I56, p. 362. See also Battaglini, "Sulle dinami jn involuzione," Atti di Napoli IV., I869; Zeuthen, Math. Ann., Band I., p. $43^{2}$. 
reciprocal, thirty of these quantities disappear, and the remainder have for their sum*

$$
2 p_{1} a_{1}^{\prime} \beta_{1}{ }^{\prime \prime}+\& c_{0}+2 p_{6} a_{6}{ }^{\prime} \beta_{6}{ }^{\prime \prime} .
$$

$\S 36$. Screw Co-ordinates.-A wrench on the screw $a$, of which the intensity is one unit, has for its components, on six co-reciprocal screws, wrenches of which the intensities may be said to constitute the co-ordinates of the screw $a$. These co-ordinates may be denoted by $\boldsymbol{a}_{1}, \& \mathrm{cc}$., $\boldsymbol{a}_{6}$.

When the co-ordinates of a screw are given, the screw itself may be thus determined. Let $\iota$ be any small quantity. Take a body in the position $A$, and impart to it successively twists about each of the screws of reference of amplitudes $\iota a_{1}, \iota a_{2}, \& c$., $\iota a_{6}$. Let the position thus attained be $B$; then the twist which would bring the body directly from $A$ to $B$ is about the required screw $\boldsymbol{a}$.

\$37. Identical Relation. - The six co-ordinates of a screw are not independent quantities, they fulfil one relation, the nature of which is suggested by the relation between three direction cosines.

When two twists are compounded by the cylindroid $(\S \mathrm{I})$, it will be observed that the amplitude of the resultant twist, as well as the direction of its screw, depend solely on the amplitudes of the given twists, and the directions of the given screws, and not at all upon either their pitches or their absolute situations. So also when any number of twists are compounded, the amplitude and direction of the resultant depend only on the amplitudes and directions of the components. We may, therefore, state the following general principle. If $n$ twists neutralize (or $n$ wrenches equilibrate) then a closed polygon

* That the work done can be represented by an expression of this kind was stated by Dr. Klein, Math. Ann., Band IV., p. 4 I3. 
of $n$ sides can be drawn, each of the sides of which is proportional to the amplitude of one of the twists (or intensity of one of the wrenches), and parallel to the corresponding screw.

Let $a_{n}, b_{n}, c_{n}$, be the direction cosines of a line parallel to any screw of reference $\omega_{n}$, and drawn through a point through which pass three rectangular axes.

Then since a wrench of one unit on $a$ has components of intensities $a_{1}$, \&c. $a_{6}$, we must have

$$
\left(a_{1} a_{1}+\& c .+a_{6} a_{6}\right)^{2}+\left(b_{1} a_{1}+\& c . b_{6} a_{6}\right)^{2}+\left(c_{1} a_{1} \& c .+c_{6} a_{6}\right)^{2}=\mathrm{I} \text {, }
$$

whence

$$
\Sigma a_{1}^{2}+2 \Sigma a_{1} a_{2} \cos \left(\omega_{1} \omega_{2}\right)=\mathrm{I},
$$

if we denote by $\cos \left(\omega_{1} \omega_{2}\right)$ the cosine of the angle between two intersecting lines parallel to $\omega_{1}$ and $\omega_{2}$.

$\S 38$. Calculation of Co-ordinates.-We must conceive the formation of a table of triple entry from which the virtual coefficient of any pair of screws may be ascertained. The three arguments will be the angle between the two screws, the perpendicular distance, and the sum of the pitches. These arguments having been ascertained by ordinary measurement of lines and angles, the virtual coefficient can be extracted from the tables.

Let $\boldsymbol{a}$ be a screw, of which the co-ordinates are to be determined. The work done against the unit wrench on $\boldsymbol{a}$ by a twist of amplitude $\omega_{1}^{\prime}$ about the screw $\omega_{1}$ is

$$
2 \omega_{1}^{\prime} \pi_{\alpha \omega_{1}}
$$

but this must equal the work done by the same twist against a wrench of intensity $a_{1}$ on the screw $\omega_{1}$, whence

$$
2 p_{1} a_{1} \omega_{1}^{\prime}=2 \omega_{1}^{\prime} \pi_{a \omega_{1}}
$$

or

$$
a_{1}=\frac{\omega_{a \omega_{1}}}{p_{2}}
$$


Thus, to compute each co-ordinate $\boldsymbol{a}_{n}$, it is only necessary to ascertain from the tables half the virtual coefficient between $a$ and $\omega_{n}$ and to divide this quantity by $p_{n}$.

$\S 39$. The Virtual Coefficient between two screws may be expressed with great simplicity by the aid of screw co-ordinates.

The components of a twist of amplitude $\boldsymbol{a}^{\prime}$ are of amplitudes $\boldsymbol{a}^{\prime} \boldsymbol{a}_{1}, \&$ c. $\boldsymbol{a}^{\prime} \boldsymbol{a}_{6}$.

The components of a wrench of intensity $\beta^{\prime \prime}$ are of intensities $\beta^{\prime \prime} \beta_{1} \&$ c. $\beta^{\prime \prime} \beta_{6}$.

Comparing these expressions with $\S 34$, we see that

$$
\alpha_{n}{ }^{\prime}=\alpha^{\prime} \alpha_{n}, \quad \beta_{n}{ }^{\prime \prime}=\beta^{\prime \prime} \beta_{n},
$$

and the expression for the work done by the twist about $a$, against the wrench on $\beta$, is

$$
a^{\prime} \beta^{\prime \prime}\left[2 p_{1} \alpha_{1} \beta_{1}+, \& \text { c., }+2 p_{6} a_{6} \beta_{6}\right] \text {. }
$$

The quantity inside the bracket is the virtual coefficient, whence we deduce the important expression

$$
\varpi_{\alpha \beta}=\Sigma p_{1} \alpha_{1} \beta_{1} \text {. }
$$

Since $\alpha$ and $\beta$ enter symmetrically into this expression, we are again reminded of the reciprocal character of the virtual coefficient.

$\S 40$. The Pitch of a screw is at once expressed in terms of its co-ordinates, for the virtual coefficient of two coincident screws being double the pitch, we have

$$
p_{a}=\Sigma p_{1} a_{1}{ }^{2} \text {. }
$$

$\S 4$ I. Screw Reciprocal to five Screws.-We can determine the co-ordinates of the single screw $\rho$, which is reciprocal to five given screws, $a, \beta, \gamma, \delta, \varepsilon$. ( $(27)$.

The quantities $\rho_{1}, \&$ c., $\rho_{6}$, must satisfy the condition

$$
\Sigma p_{1} \rho_{1} a_{1}=0 \text {, }
$$


and four similar equations; hence $p_{n} \rho_{n}$ is proportional to the determinant obtained by omitting the $n^{\text {th }}$ column from the matrix.

$$
\left|\begin{array}{llllll}
a_{1}, & a_{2}, & a_{3}, & \alpha_{4}, & a_{5}, & a_{6}, \\
\beta_{1}, & \beta_{2}, & \beta_{3}, & \beta_{4}, & \beta_{5}, & \beta_{6}, \\
\gamma_{1}, & \gamma_{2}, & \gamma_{3}, & \gamma_{4}, & \gamma_{5}, & \gamma_{6}, \\
\delta_{1}, & \delta_{2}, & \delta_{3}, & \delta_{4}, & \delta_{5}, & \delta_{6}, \\
\varepsilon_{1}, & \varepsilon_{2}, & \varepsilon_{3}, & \varepsilon_{4}, & \varepsilon_{5}, & \varepsilon_{7},
\end{array}\right|
$$

The relative values of $\rho_{1}, \&$ c., $\rho_{6}$, being thus found, the absolute values are given by $\S 37$.

The condition that six screws have a common reciprocal screw is expressed by the evanescence of a determinant, which may be compared with the condition that three straight lines be coplanar, of which the direction cosines are given.

$\S 42$. Co-ordinates of a Screw on a Cylindroid. - We may define the screw $\theta$ on the cylindroid by the angle $l$, which it makes with the screw $a$ on the axis of $x$. Since a wrench of unit intensity on $\theta$ has components of intensities $\cos l$ and $\sin l$ on $\boldsymbol{a}$ and $\beta$ ( $\S$ I 7 ), and since each of these components may be resolved into six wrenches on any six co-reciprocal screws, we must have $(\$ 36)$

$$
\theta_{n}=a_{n} \cos l+\beta_{n} \sin l \text {. }
$$

From this expression we can find the pitch of $\theta$ :

for we have

$$
p_{\theta}=\Sigma p_{1}\left(a_{1} \cos l+\beta_{1} \sin l\right)^{2}
$$

whence expanding and observing that as $a$ and $\beta$ are rèciprocal $\Sigma p_{1} a_{1} \beta_{1}=0$ and also that $\Sigma p_{1} a_{1}{ }^{2}=p_{a}$ and $\Sigma p_{1} \beta_{1}{ }^{2}=p_{\beta}$, we have the expression already given $(\$ 20)$ viz.

$$
p_{0}=p_{\alpha} \cos ^{2} l+p_{\beta} \sin ^{2} l
$$


If two screws, $\theta$ and $\phi$, upon the cylindroid, are reciprocal, then ( $m$ being the defining angle of $\phi$ ),

$$
\Sigma p_{1}\left(a_{1} \cos l+\beta_{1} \sin l\right)\left(a_{1} \cos m+\beta_{1} \sin m\right)=0,
$$

or, $\quad p_{a} \cos l \cos m+p_{\beta} \sin l \sin m=0$.

Comparing this with $\S 20$, we have the following useful theorem :-

Any two reciprocal screws on a cylindroid are parallel to conjugate diameters* of the pitch conic.

Since the sum or difference of the squares of two conjugate diameters is constant $\dagger$ according as the pitch conic is an Ellipse or a Hyperbola, we see that the sum or difference of the reciprocals of the pitches of two reciprocal screws on a cylindroid is constant according as the pitch conic is an Ellipse or a Hyperbola.

* Salmon, Conic Sections, 3rd Ed., page I29.

+ Do. page 153 . 


\section{CHAPTER V.}

GENERAL CONSIDERATIONS ON THE EQUILIBRIUM OF A RIGID BODY.

$\S 43$. The Screw Complex.-To specify with precision the nature of the freedom enjoyed by a rigid body, it is necessary to ascertain all the screws about which the constraints will permit the body to be twisted. When the attempt has been made for every screw in space, the results will give us all the information conceivable with reference to the freedom of the body, and also with reference to the constraints by which the movement may be hampered.

Suppose that $n$ screws $A_{1}, \& c ., A_{n}$ have been found by these trials, about each of which the body can receive a twist. It is evident, without further trial, that twisting about an infinite number of other screws must also be possible $(n>1)$ : for suppose the body receive any $n$ twists about $A_{1}, \&$ c., $A_{n}$ the position attained could have been reached by a twist about some single screw $A$. It follows that the body must be free to twist about $A$. Now since the amplitudes of the $n$ twists may have any magnitude (each not exceeding an infinitely small quantity), $A$ is merely one of an infinite number of screws, about which twisting must be possible. All these screws, together with $A_{1}, \& c ., A_{n}$, we call a screw complex of the $n^{\text {th }}$ order.

If it be found that the body cannot be twisted about any screw which does not belong to the screw complex of the $n^{\text {th }}$ order, then the body is said to have freedom of the $n^{\text {th }}$ order. It may be necessary to remark that $A_{1}, \& \mathrm{c}$., $A_{n}$, must not be themselves members of a screw complex of order lower than $n$. If this were the case, the screws 
about which the body could be twisted would only consist of the members of that lower screw complex.

Since the amplitudes of the $n$ twists about $A_{1}, \ldots A_{n}$ are [arbitrary, it might be thought that there are $n$ disposable quantities in the selection of a screw $S$ from a screw complex of the $n^{\text {th }}$ order. It is, however, obvious from $\S$ I 7 that the determination of the position and pitch of $S$ depends onlyupon the ratios of the amplitudes of the twists about $A_{1}, \ldots A_{n}$ and hence in the selection of $a$ screw from the screw complex of the $n^{\text {th }}$ order, we have $n-I$ disposable quantities.

$\S 44$. Constraints. - An essential feature of a system of constraints consists in the number of independent quantities which are necessary to specify the position of the body when displaced in conformity with the requirements of the constraints. That number which cannot be less than one, nor greater than six, is the order of the freedom. To each of the six orders of freedom a certain type of screw complex is appropriate.

The study of the six types of screw complex as here defined is a problem of kinematics, but the statical and kinematical properties of screws are so interwoven that we derive great advantages by not attempting to relegate the statics and kinematics to different chapters. We shall not require any further mention of the constraints. Every conceivable condition of constraints must have been included when the six screw complexes are discussed in their most general form. Nor does it come within our scope, except on rare occasions, to specialize the enunciation of any problem, further than by mentioning the order of the freedom permitted to the body.

$\S 45$. Screw Reciprocal to a Complex. - If a screw $X$ be reciprocal to $n$ screws, $A_{1}, \& c$., $A_{n}$, belonging to a screw complex of order $n$, then $X$ is reciprocal to every other screw $A$ which belongs to the same screw com- 
plex. For, by the definition of the screw complex, it appears that twists of appropriate amplitudes about $A_{1}$, \&c., $A_{n}$, would compound into a twist about $A$. It follows ( $\$ 43)$ that wrenches on $A_{1}, \&$ c., $A_{n}$, of appropriate intensities ( $\$ 32)$ compound into a wrench on $A$. Suppose these wrenches on $A_{1}, \& c ., A_{n}$, were applied to a body only free to twist about $X$, then since $X$ is reciprocal to $A_{1}$, \&c., $A_{n}$, the equilibrium of the body would be undisturbed. The resultant wrench on $A$ must therefore be incapable of moving the body, therefore $A$ and $X$ must be reciprocal.

\$46. The Reciprocal Screw Complex.-All the screws which are reciprocal to a screw complex $P$ of order $k$ constitute a screw complex $Q$ of order $6-k$. This important theorem is thus proved:-

Since only one condition is necessary for a pair of screws to be reciprocal, it follows, from the last section, that if a screw $X$ be reciprocal to $P$ it will fulfil $k$ conditions. The screw $X$ has, therefore, $5-k$ elements still disposable, and consequently $(k<5)$ an infinite number of screws $Q$ can be found which are reciprocal to the screw complex $P$. The theory of reciprocal screws will now prove that $Q$ must really be a screw complex of order $6-k$. In the first place it is manifest that $Q$ must be a screw complex of some order, for, in general, if a body be capable of twisting about even six screws, it must be perfectly free. Here, however, if a body were able to twist about the infinite number of screws embodied in $Q$, it would still not be free, because it would remain in equilibrium, though acted upon by a wrench about any screw of $P$. If follows that $Q$ can only denote the collection of screws about which a body can twist which has some definite order of freedom. It is easily seen that that number must be $6-k$, for the number of constants disposable in the selection of a screw belong- 
ing to a screw complex is one less than the order of the complex $(\$ 38)$. But.we have seen that the constants disposable in the selection of $X$ are $5-k$, and, therefore, $Q$ must be a screw complex of order $6-k$.

We thus see, that to any screw complex $P$ of order $k$ belongs a reciprocal screw complex $Q$ of order $6-k$. Every screw of $P$ is reciprocal to all the screws of $Q$, and vice versâ. This theorem provides us with a definite test as to whether any given screw $a$ is a member of the screw complex $P$. Construct any $6-k$ screws of the reciprocal system. If then $\boldsymbol{a}$ be reciprocal to these $6-k$ screws, $\boldsymbol{a}$ must belong to $P$. We thus have $6-k$ conditions to be satisfied by any screw when a member of a screw complex of order $k$.

$\S 47$. Equilibrium.-If the screw complex $P$ expresses the freedom of a rigid body, then the body will remain in equilibrium though acted upon by a wrench on any screw of the reciprocal screw complex $Q$. This is, perhaps, the most general theorem which can be enunciated with respect to the equilibrium of a rigid body. This theorem is thus proved:- Suppose a wrench to act on a screw $\eta$ belonging to $Q$. If the body does not continue at rest, let it commence to twist about $\boldsymbol{a}$. We thus have a wrench about $\eta$ disturbing a body which twists about $a$, but this is impossible, because $a$ and $\eta$ are reciprocal.

In the same manner it may be shown that a body which is free to twist about all the screws of $Q$ will not be disturbed by a wrench about any screw of $P$. Thus, of two reciprocal screw complexes, each expresses the locus of a wrench which is unable to disturb a body free to twist about any screw of the other.

$\S 48$. Reaction of Constraints. - It also follows that the reactions of the constraints by which the movements of a body are confined to twists about the screws of 
a complex $P$ can only be wrenches on the reciprocal screw complex $Q$, for the reactions of the constraints are only manifested by the success with which they resist. the efforts of certain wrenches to disturb the equilibrium of the body.

$\S 49$. Parameters of a Screw Complex.-We next consider the question as to how many parameters are required in order to specify completely a screw complex of the $n^{\text {th }}$ order. Since the complex is defined when $n$ screws are given, and since five data are required. for each screw, it might be thought that $5 n$ parameters. would be necessary. It must be observed, however, that the given $5 n$ data suffice not only for the purpose of defining the screw complex but also for pointing out $n$ : special screws upon the screw complex, and as the pointing out of each screw on the complex requires $n-\mathbf{I}$ quantities. $(\$ 38)$, it follows that the number of parameters. actually required to define the complex is only

$$
5 n-n(n-\mathrm{I})=n(6-n) \text {. }
$$

This result has a very significant meaning in connexion with the theory of reciprocal screw complexes $P$. and $Q$. Assuming that the order of $P$ is $n$, the order of $Q$ is $6-n$; but the expression $n(6-n)$ is unaltered by changing $n$ into $6-n$. It follows that the number of parameters necessary to specify a screw complex is identical with the number necessary to specify its reciprocal screw complex. This remark is chiefly of importance in connexion with the complexes of the fourth and fifth orders, which are respectively the reciprocal complexes of a cylindroid and a single screw. We are now assured that a collection of all the screws which are reciprocal to an arbitrary cylindroid can be nothing less than a screw complex of the fourth order in its most general type, and also, that all the screws in space which 
are reciprocal to a single screw must form the most general type of a screw complex of the fifth order.

$\S 50$. Applications of Co-ordinates. - If the co-ordinates of a screw satisfy $n$ linear equations, the screw must belong to a screw complex of the order $6-n$. Let $\eta$ be the screw, and let one of the equations be

$$
A_{1} \eta_{1}+\text {, \&c., }+A_{6} \eta_{6}=0 \text {, }
$$

whence $\eta$ must be reciprocal to the screw whose co-ordinates are proportional to

$$
\frac{A_{1}}{p_{1}}, \& \text { c., } \frac{A_{6}}{p_{6}},(\S 39) \text {. }
$$

It follows that $\eta$ must be reciprocal to $n$ screws, and therefore belong to a screw complex of order $6-n$.

Let $a, \beta, \gamma, \delta$ be four screws about which a body receives twists of amplitudes $a^{\prime}, \beta^{\prime}, \gamma^{\prime}, \delta^{\prime}$. It is required to find the co-ordinates of the screw $\rho$ and the amplitude $\rho^{\prime}$ of a twistabout $\rho$ which will produce the same effect as the fourgiven twists. We haveseen $(\$ 39)$ that the twist about any screw $a$, may be resolved in one way into six twists of amplitudes $\boldsymbol{a}^{\prime} \boldsymbol{a}_{1}, \ldots \boldsymbol{a}^{\prime} \boldsymbol{a}_{6}$, on the six screws of reference; we must therefore have

$$
\begin{array}{r}
\rho^{\prime} \rho_{1}=a^{\prime} a_{1}+\beta^{\prime} \beta_{1}+\gamma^{\prime} \gamma_{1}+\delta^{\prime} \delta_{1} \\
\quad \& c ., \quad \& c . \\
\rho^{\prime} . \rho_{6}=a^{\prime} a_{6}+\beta^{\prime} \beta_{6}+\gamma^{\prime} \gamma_{6}+\delta^{\prime} \delta_{6}
\end{array}
$$

whence $\rho^{\prime}$ and $\rho_{1}, \ldots \rho_{8}$ can be found $(\$ 37)$.

A similar process will determine the co-ordinates of the resultant of any number of twists, and it follows from $\$$ I 5 that the resultant of any number of wrenches is to be found by equations of the same form. In ordinary mechanics, the conditions of equilibrium of any number of forcesare six, viz., that each of the three forces, and each of the three couples to which the system is equivalent shall vanish. In the present theory the conditions are 
likewise six, viz., that the intensity of each of the six wrenches on the screws of reference to which the given system is equivalent shall be zero.

Any screw will belong to a complex of the $n^{\text {th }}$ order if it be reciprocal to $6-n$ independent screws; it follows that $6-n$ conditions must be fulfilled when $n+\mathrm{I}$ screws belong to a screw complex of the $n^{\text {th }}$ order.

To determine these conditions we take the case of $n=3$, though the process is obviously general. Let $a, \beta$, $\gamma, \delta$ be the four screws, then since twists of amplitudes $a^{\prime}, \beta^{\prime}, \gamma^{\prime}, \delta^{\prime}$ neutralise, we must have $\rho^{\prime}$ zero and hence the six equations

$$
\begin{array}{r}
a^{\prime} a_{1}+\beta^{\prime} \beta_{1}+\gamma^{\prime} \gamma_{1}+\delta^{\prime} \delta_{1}=0 \\
\& c . \\
a^{\prime} a_{6}+\beta^{\prime} \beta_{6}+\gamma^{\prime} \gamma_{6}+\delta^{\prime} \delta_{6}=0 ;
\end{array}
$$

from any four of these equations the quantities $a^{\prime}, \beta^{\prime}, \gamma^{\prime}$, $\delta^{\prime}$ can be eliminated, and the result will be one of the required conditions.

It is noticeable that the $6-n$ conditions are often presented in the evanescence of a single function, just as the evanescence of the sine of an angle between a pair of straight lines embodies the two conditions necessary that the direction cosines of the lines coincide. The function is suggested by the following considerations :If $n+2$ screws belong toa screw complex of the $(n+I)^{\text {th }}$ order, twists of appropriate amplitudes about the screws neutralise. The amplitude of the twist about any one screw must be proportional to a function of the co-ordinates of all the other screws ; this is evident, because if one amplitude were ascertained to be zero, the remaining screws must belong to a complex of the $n^{\text {th }}$ order. We thus see that the evanescence of one function must afford all that is necessary for $n+I$ screws to belong to a screw complex of the $n^{\text {th }}$ order. 


\section{CHAPTER VI.}

THE PRINCIPAL SCREWS OF INERTIA.

$\$ 5$. Introduction.-If a rigid body be free to rotate about a fixed point, then it is well known that an impulsive couple in a plane perpendicular to one of the principal axes which can be drawn through the point will make the body commence to rotate about that axis. Suppose that on one of the principal axes lay a screw $\eta$ with a very small pitch, then a twisting motion about $\eta$ would closely resemble a simple rotation about the corresponding axis. An impulsive wrench on $\eta$ will, when united with the reaction of the fixed point, reduce to a couple in a plane perpendicular to the axis. If we now suppose the pitch of $\eta$ to be evanescent, we may still assert that an impulsive wrench on $\boldsymbol{\eta}$ of very great intensity will cause the body, if previously quiescent, to commence to twist about $\eta$.

We have stated a familiar property of the principal axes in this indirect manner, for the purpose of showing that it is merely an extreme case for a body with freedom of the third order of the following general theorem :-

If a quiescent rigid body have freedom of the $n^{\text {th }}$ order, then $n$ screws can always be found (but not more than $n$ ), such that if the body receive an impulsive wrench on any one of these screws, the body will commence to twist about the same screw.

These $n$ screws are of great significance in the present method of studying Dynamics, and they may be termed the principal screws of inertia. In the present chapter we shall prove the general theorem just stated, 
while in the chapters on the special orders of freedom we shall show how the principal screws of inertia are to be determined for each case.

$\S 52$. Screws of Reference.-We have now to define the group of six co-reciprocal screws $(\$ 28)$ which are peculiarly adapted to serve as the screws of reference in Kinetic investigations. Let $O$ be the centre of inertia of the rigid body, and let $O A, O B, O C$ be the three principal axes through $O$, while $a, b, c$ are the corresponding radii of gyration. Then two screws along $O A$, viz. : $\omega_{1}$, $\omega_{2}$, with pitches $+a,-a$; two screws along $O B$, viz.: $\omega_{3}$, $\omega_{4}$, with pitches $+b,-b$, and two along $O C$, viz. : $\omega_{5}, \omega_{6}$, with pitches $+c,-c$, are the co-reciprocal group which we shall employ. For convenience in writing the formulæ, we shall often use $p_{1}, \& c . p_{6}$, to denote the pitches as before.

We shall now prove that the six screws thus defined are the principal screws of inertia of a free rigid body. Let the mass of the body be $M$, and let an impulsive wrench on $\omega_{1}$ act for a short time $t$. The intensity of this wrench is $\omega_{1}^{\prime \prime}$, and the moment of the couple is $a \omega_{1}^{\prime \prime}$. We now consider the effect of the two portions of the wrench separately. The effect of the force $\omega_{1}{ }^{\prime \prime}$ is to give the body a velocity of translation parallel to $O A$ and equal to $\frac{t}{M} \omega_{1}^{\prime \prime}$. By the property of the principal axes the effect of the couple will be to impart an angular velocity $\dot{\omega}_{1}^{\prime}$ about the axis $O A$. This angular velocity is easily determined. The effective force which must have acted upon a particle $d m$ at a perpendicular distance $r$ from $O A$ is $\frac{r \dot{\omega}_{1}^{\prime}}{t} d m$. The sum of the moments of all these forces is $M a^{2} \frac{\dot{\omega}_{1}^{\prime}}{t}$. This quantity must equal 
the moment of the given couple or

whence

$$
\begin{aligned}
& M a^{2} \frac{\dot{\omega}_{1}^{\prime}}{t}=a \omega_{1}^{\prime \prime} \\
& \omega_{1}^{\prime}=\frac{t}{a M} \omega_{1}^{\prime \prime} .
\end{aligned}
$$

The effect of an impulsive wrench on $\omega_{1}$ is, therefore, to give the body a velocity of translation parallel to $O A$, and equal to $\frac{t}{M} \omega_{1}^{\prime \prime}$, and also a velocity of rotation about $O A$ equal to $\frac{t}{a M} \omega_{1}^{\prime \prime}$. These movements unite to form a twisting motion about a screw on $O A$, of which the pitch is found by dividing the velocity of translation by the velocity of rotation to be equal to $a$, this being the pitch of $\omega_{1}$, proves that an impulsive wrench on $\omega_{1}$ will make the body commence to twist about $\omega_{1}$, and that, therefore, $\omega_{1}$ is a principal screw of inertia. Similar reasoning applies to the remaining five screws.

\$53. Impulsive Screws and Instantaneous Screws.-If a free quiescent rigid body receive an impulsive wrench on a screw $\eta$, the body will immediately commence to twist about an instantaneous screw $a$. The co-ordinates of $\boldsymbol{a}$ being given, it is required to determine the coordinates of $\eta$.

The impulsive wrench on $\eta$ of intensity $\eta^{\prime \prime}$ is to be decomposed into components of intensities $\eta^{\prime \prime} \eta_{1}$, \&c. $\eta^{\prime \prime} \eta_{6}$ on $\omega_{1}$, \&c $\omega_{6}$. The component on $\omega_{n}$ will generate a twist velocity about $\omega_{n}$ amounting to

$$
\eta^{\prime \prime} \frac{\eta_{n}}{p_{n} M} t
$$

but if $\dot{a}^{\prime}$ be the twist velocity about $a$ which is finally produced, the expression just written must be equal to $\dot{\boldsymbol{a}}^{\prime} \boldsymbol{a}_{n}$, and hence we have the following useful result :-

If the co-ordinates of the instantaneous screw be propor- 
tional to $a_{1}, E c . a_{6}$, then the co-ordinates of the corresponding impulsive screw are proportional to $p_{1} a_{1}, \delta_{c}$. $p_{6} a_{6}$.

$\$ 54$. Conjugate Screws of Inertia.-If $\alpha$ and $\beta$ be twoinstantaneous screws, and if $\eta$ and $\xi$ be the corresponding impulsive screws, then when $a$ is reciprocal to $\xi$ wemust have $\beta$ reciprocal to $\eta$. We shall first suppose the body to be perfectly free.

The co-ordinates of $\xi$ are proportional to $p_{1} \beta_{1}, \&$ c. $p_{6} \beta_{6}$, hence the condition that $\boldsymbol{a}$ and $\boldsymbol{\xi}$ are reciprocal ( $(34)$ is

$$
p_{1}{ }^{2} a_{1} \beta_{1}+\& \mathrm{cc} .+p_{6}{ }^{2} a_{6} \beta_{6}=0 .
$$

But this is precisely the equation which we should have found by expressing the condition that $\beta$ and $\eta$ were reciprocal.

When this relation is fulfilled, the screws $a$ and $\beta$ are said to be conjugate screws of inertia.

We shall now show that this theorem will still remain true even if the body be only partially free. When the body receives an impulsive wrench on $\xi$ there is an impulsive reaction of the constraints on a screw $\mu$. The effect on the body is, therefore, the same as if it had been free, but had received an impulsive wrench of which the component on $\omega_{1}$ had the intensity $\xi^{\prime \prime} \xi_{1}+\mu^{\prime \prime} \mu_{1}$; hence, $h$ being a constant, we have

$$
\begin{gathered}
\xi^{\prime \prime} \xi_{1}+\mu^{\prime \prime} \mu_{1}=h p_{1} \beta_{1} \\
\& c . \quad \& c . \\
\xi^{\prime \prime} \xi_{6}+\mu^{\prime \prime} \mu_{6}=h p_{6} \beta_{6},
\end{gathered}
$$

multiplying the first of these equations by $p_{1} a_{1}$, the second by $p_{2} a_{2}, \&$ c., adding the six products, and remembering that $a$ and $\xi$ are reciprocal by hypothesis, while $a$ and $\mu$ are reciprocal, by the nature of the reactions of the constraints $(\S 43)$, we have, as before

$$
p_{1}{ }^{2} \alpha_{1} \beta_{1}+\& \mathrm{c} .+p_{6}{ }^{2} a_{6} \beta_{6}=0 \text {. }
$$


Precisely the same condition must be satisfied when $\beta$ and $\eta$ are reciprocal, and hence the general property of conjugate screws of inertia is true, whether the body be free or constrained in any way.

$\S 55$. The Determination of the Impulsive Screw, corresponding to a given instantaneous screw, is a definite problem when the body is perfectly free. If, however, the body be constrained, we shall show that any screw selected from a certain screw complex will fulfil the required condition.

Let $B_{1}$, \&c. $B_{6-n}$ be $6-n$ screws selected from the screw complex which is reciprocal to that corresponding to the freedom of the $n^{\text {th }}$ order possessed by the rigid body. Let $S$ be the screw about which the body is to twist. Let $X$ be any screw, an impulsive wrench about which would make the body twist about $S$; then any screw $Y$ belonging to the screw-complex of the $(7-n)^{\text {th }}$ order, specified by the screws, $X, B_{1}, \&$ c. $B_{6-n}$ is an impulsive screw, corresponding to $S$ as an instantaneous screw. For the wrench on $Y$ may be resolved into $7-n$ wrenches on $X, B_{1}, \&$ c. $B_{6-n}$; of these, all but the first are instantly destroyed by the reaction of the constraints, so that the wrench on $Y$ is practically equivalent to the wrench on $X$, which, by hypothesis, will make the body twist about $S$.

For example, if the body had freedom of the fifth order, then an impulsive wrench on any screw on a certain cylindroid will make the body commence to twist about a given screw.

If a body have freedom of the third order, then the "locus" of an impulsive wrench which would make the body twist about a given screw consists of all the screws in space which are reciprocal to a certain cylindroid.

$\S 56$. System of Conjugate Screws of Inertia.-We shall now show that from the screw-complex of the $n^{\text {th }}$ order $P$, 
which expresses the freedom of the rigid body, $n$ screws can be selected so that every pair of them are conjugate screws of inertia $(\$ 54)$. Let $B_{1}, \&$ c. $B_{6-n}$ be $(6-n)$ screws defining the reciprocal screw-complex. Let $A_{1}$ be any screw belonging to $P$. Then in the choice of $A A_{1}$ we have $n-\mathrm{I}$ arbitrary quantities. Let $T_{1}$ be any impulsive screw corresponding to $A_{1}$ as an instantaneous screw. Choose $A_{2}$ reciprocal to $I_{1}, B_{1}$, \&c. $B_{6-n}$, then $A_{1}$ and $A_{2}$ are conjugate screws, and in the choice of the latter we have $n-2$ arbitrary quantities. Let $I_{2}$ be any impulsive screw corresponding to $A_{2}$ as an instantaneous screw. Choose $A_{3}$ reciprocal to $I_{1}, I_{2}, B_{1}$, \&c. $B_{6-n}$, and proceed thus until $A_{n}$ has been attained, then each pair of the group $A_{1}$, \&c. $A_{n}$ are conjugate screws of inertia. The number of quantities which remain arbitrary in the choice of such a group amount to

$$
n-\mathrm{I}+n-2+\& \mathrm{c} .+\mathrm{I}=\frac{n(n-\mathrm{I})}{2}
$$

or exactly half the total number of arbitrary constants in the selection of any $n$ screws from a complex of the $n^{\text {th }}$ order.

$\S 57$. Principal Screws of Inertia.-It is the object of this section to show that it is always possible to select from the screw-complex of the $n^{\text {th }}$ order expressing the freedom of a rigid body, one group of $n$ screws, of which every pair are both conjugate and reciprocal, and that these constitute the principal screws of inertia ( $\$ 5 \mathrm{I}$ ).

To prove this, it is sufficient to show that when the remaining half of the arbitrary constants ( $\$ 56$ ) have been suitably disposed, then the group of $n$ screws besides being conjugate will be co-reciprocal. Choose $A_{1}$ reciprocal to $B_{1}, \&$ c. $B_{6-n}$, with $n-\mathrm{I}$ arbitrary quantities ; $A_{2}$ reciprocal to $A_{1}, B_{1}$, \&c. $B_{n-1}$, with $n-2$ arbitrary quantities, and so on, then the total number of arbitrary 
quantities in the choice of $n$ co-reciprocal screws from a complex of the $n^{\text {th }}$ order is

$$
n-\mathrm{I}+n-2 \ldots+\mathrm{I}=\frac{n(n-1)}{2} \text {. }
$$

Hence, by suitable disposition of the $n(n-\mathrm{x})$ constants we can find one group of $n$ screws which are both conjugate and co-reciprocal.

We have now to show that these $n$ screws are really the principal screws of inertia ( $(5 \mathrm{I})$. $\downarrow W$ We shall state the argument for the freedom of the third order, the argument for any other order being precisely similar.

Let $A_{1}, A_{2}, A_{3}$, be the three conjugate and co-reciprocal screws which can be selected from a complex of the third order. Let $B_{1}, B_{2}, B_{3}$ be any three screws belonging to the reciprocal screw-complex. Let $R_{1}, R_{2}, R_{3}$ be any three impulsive screws corresponding respectively to $A_{1}, A_{2}, A_{3}$ as instantaneous screws.

An impulsive wrench on any screw belonging to the screw-complex of the $4^{\text {th }}$ order defined by $R_{1}, B_{1}, B_{2}, B_{3}$ will make the body twist about $A_{1}(\S 55)$, but the screws of such a complex are reciprocal to $A_{2}$ and $A_{3}$; for since $A_{1}$ and $A_{2}$ are conjugate, $R_{1}$ must be reciprocal to $A_{2}$ ( $\$ 54)$, and also to $A_{3}$, since $A_{1}$ and $A_{3}$ are conjugate. It follows from this that an impulsive wrench on any screw reciprocal to $A_{2}$ and $A_{3}$ will make the body commence to twist about $A_{1}$, but $A_{1}$ is itself reciprocal to $A_{2}$ and $A_{3}$, and hence an impulsive wrench on $A_{1}$ will make the body commence to twist about $A_{1}$. Hence $A_{1}$ and also $A_{2}$ and $A_{3}$ are principal screws of inertia.

We shall now show that with the exception of the $n$ screws here determined, no other screw possesses the property in question. Suppose another screw $S$ were to possess this property. Decompose the wrench on $S$ into $n$ wrenches of intensities $S_{1}^{\prime \prime}$, \&c. $S_{n}^{\prime \prime}$ on $A_{1}$, \&c. $A_{n}$, this must be possible, because if the body is to be capable of 
twisting about $S$ this screw must belong to the complex specified by $A_{1}, \&$ c. $A_{n}$. The $n$ impulsive wrenches on $A_{1}$, \&c. $A_{n}$ will produce twisting motions about the same screws, but these twisting motions are to compound into a twisting motion on $S$. It follows that the component twist velocities $S_{1}{ }^{\prime}$, \&c. $S_{n}{ }^{\prime}$ must be proportional to the intensities $S_{1}^{\prime \prime}$, \&c. $S_{n}{ }^{\prime \prime}$. But if this were the case, then every screw of the complex would be a principal screw of inertia; for let $X$ be any impulsive screw, and suppose that $Y$ is the corresponding instantaneous screw, the components of $X$ on $A_{1}, \&$ c. $A_{n}$, have intensities $X_{1}^{\prime \prime}$, \&c. $X_{n}^{\prime \prime}$, these will generate twist velocities equal to

$$
\frac{S_{1}^{\prime}}{S_{1}^{\prime \prime}} X_{1}^{\prime \prime}, \& c \cdot \frac{S_{n}^{\prime}}{S_{n}^{\prime \prime}} X_{n}^{\prime \prime},
$$

and these quantities must equal the components of the twist velocity about $Y$. But the ratios

$$
\frac{S_{1}^{\prime}}{S_{1}^{\prime \prime}} \text { \&c. } \frac{S_{n}^{\prime}}{S_{n}{ }^{\prime \prime}}
$$

are all equal, and hence the twist velocities of the components on the screws of reference of the twisting motion about $Y$ must be proportional to the intensities of the components on the same screws of reference of the wrench on $X$. Remembering that twisting motions and wrenches are compounded by the same rules, it follows that $Y$ and $X$ must be identical.

As it is not generally true that all the screws of the complex defining the freedom possess the property enjoyed by a principal screw of inertia, it follows that the number of principal screws of inertia must be generally equal to the order of the freedom.

$\S 58$. Kinetic Energy. - The twisting motion of a rigid body with freedom of the $n^{\text {th }}$ order may be completely specified by the twist velocities of the components of the 
twisting motion on any $n$ screws of the complex defining the freedom. If the screws of reference be a set of conjugate screws of inertia, the expression for the kinetic energy of the body consists of $n$ square terms. This will now be proved.

If a free or constrained rigid body be at rest in a position $A$, and if the body receive an impulsive wrench, the body will commence to twist about a screw $a$ with a kinetic energy $E_{\alpha}$. Let us now suppose that a second impulsive wrench acts upon the body on a screw $\mu$, and that if the body had been at rest in the position $A$, it would have commenced to twist about a screw $\beta$, with a kinetic energy $E_{\beta}$.

We are now to consider how the amount of energy acquired by the second impulse is affected by the circumstance that the body is then not at rest in $A$, but is moving through $A$ in consequence of the former impulse. The amount will in general differ from $E_{\beta}$, for the movement of the body may cause it to do work against the wrench on $\mu$ during the short time that it acts, so that not only will the body thus expend some of the kinetic energy which it previously possessed, but the efficiency of the impulsive wrench on $\mu$ will be diminished. Under other circumstances the motion through $A$ might be of such a character that the impulsive wrench on $\mu$ acting for a given time would impart to the body a larger amount of kinetic energy than if the body were at rest. Between these two cases must lie the intermediate one in which the kinetic energy imparted is precisely the same as if the body had been at rest. It is obvious that this will happen if each point of the body at which the forces of the impulsive wrench are applied be moving in a direction perpendicular to the corresponding force, or more generally if the screw $\boldsymbol{a}$ about which the body is twisting be reciprocal to $\mu$. When this is the case 
$\boldsymbol{a}$ and $\beta$ must be conjugate screws of inertia ( $\S 54)$, and hence we infer the following theorem :-

If the kinetic energy of a body twisting about a screw $\boldsymbol{a}$ with a certain twist velocity be $E_{\boldsymbol{a}}$, and if the kinetic energy of the same body twisting about a screw $\beta$ with a certain twist velocity be $E_{\beta}$, then when the body has a motion compounded of the two twisting movements, its kinetic energy will amount to $E_{a}+E_{\beta}$ provided that $a$ and $\beta$ are conjugate screws of inertia.

Since this result may be extended to any number of conjugate screws of inertia, and since the terms $E_{a}$, \&c., are essentially positive, the required theorem has been proved.

§59. Expression for Kinetic Energy.-If a rigid body have a twisting motion about a screw $a$, with a twist velocity $a^{\prime}$, what is the expression of its kinetic energy in terms of the co-ordinates of $\boldsymbol{a}$ ?

We adopt as the unit of force that force which acting upon the unit of mass for the unit of time will give the body a velocity which would carry it over the unit of distance in the unit of time. The unit of energy is the work done by the unit force in moving over the unit distance. If, therefore, a body of mass $w$ have a movement of translation with a velocity $v$ its kinetic energy expressed in these units is $\frac{1}{2} w v^{2}$.

The movement is to be decomposed into twisting motions about the screws of reference $\omega_{1}$, \&c. $\omega_{6}$, the twist velocity of the component on $\omega_{m}$ being $\ddot{a}^{\prime} a_{m}$. One constituent of the twisting motion about $\omega_{m}$ consists of a velocity of translation equal to $a^{\prime} p_{m} \alpha_{m}$, and on this account the body has a kinetic energy equal to $\frac{1}{2} M \dot{\alpha}^{\prime 2} p_{m}^{2} a_{m}^{2}$. On account of the rotation around the axis with an angular velocity $\dot{\boldsymbol{a}}^{\prime} \boldsymbol{a}_{m}$ the body has a kinetic energy equal to

$$
\frac{1}{2} a^{\prime 2} a_{m}^{2} \int r^{2} d M
$$


where $r$ denotes the perpendicular from the element $d M$ on $\omega_{m}$. Remembering that $p_{n}$ is the radius of gyration this expression also reduces to $\frac{1}{2} M a^{\prime 2} p_{m}^{2} a_{m}^{2}$, and hence the total kinetic energy of the twisting motion about $\omega_{m}$ is $M \dot{a}^{\prime 2} p_{m}^{2} a_{m}^{2}$.

We see, therefore $(\$ 58)$, that the kinetic energy due to the twisting motion about $a$ is

$$
M \dot{a}^{\prime 2}\left(p_{1}^{2} a_{1}^{2}+\& c .+p_{6}{ }^{2} a_{6}{ }^{2}\right) .
$$

The quantity inside the bracket is the square of a certain linear magnitude which is determined by the distribution of the material of the body with respect to the screw $\boldsymbol{a}$. It will facilitate the kinetic applications of the present theory to employ the symbol $u_{a}$ to denote this quantity. It is then to be understood that the kinetic energy of a body of mass $M$, animated by a twisting motion about the screw $a$ with a twist velocity $a^{\prime}$ is represented by

$$
M \dot{a}^{\prime 2} u_{a}^{2} \text {. }
$$

$\S 60$. Twist Velocity acquired by an Impulse.-A body of mass $M$, which is only free to twist about a screw $a$, is acted upon for a short time $e$ by a wrench of intensity $\eta^{\prime \prime}$ on a screw $\eta$. It is required to find the twist velocity $a^{\prime}$ which is acquired.

Let the initial reaction of the constraints consist of a wrench of intensity $\lambda^{\prime \prime}$ on a screw $\lambda$. Then the body moves as if it were free, but had been acted upon by a wrench of which the component on $\omega_{m}$ had the intensity $\eta^{\prime \prime} \eta_{m}+\lambda^{\prime \prime} \lambda_{m}$. This component would generate a velocity of translation parallel to $\omega_{m}$ and equal to $\frac{e}{M}\left(\eta^{\prime \prime} \eta_{m}+\lambda^{\prime \prime} \lambda_{m}\right)$. The twist velocity about $\omega_{m}$ produced by this component is found by dividing the velocity of translation by $p_{m}$. On the other hand, since the co-ordinates of the screw 
$\boldsymbol{a}$ are $\boldsymbol{\alpha}_{1}, \& \mathrm{c} ., \boldsymbol{\alpha}_{6}$, the twist velocity about $\omega_{m}$ may also be represented by $\dot{a}^{\prime} \boldsymbol{a}_{m}(\S 36)$, whence

$$
\dot{\boldsymbol{a}}^{\prime} \boldsymbol{a}_{m}=\frac{e}{M} \frac{\eta^{\prime \prime} \eta_{m}+\lambda^{\prime \prime} \lambda_{m}}{p_{m}} .
$$

If we multiply this equation by $p_{m}^{2} a_{m}$, add the six equations found by giving $m$ all values from $\mathrm{I}$ to 6 , and remember that $\boldsymbol{a}$ and $\lambda$ are reciprocal, we have ( $\S 39$,)

$$
\dot{a}^{\prime} u_{\alpha}^{2}=\frac{e}{M} \eta^{\prime \prime} \varpi_{\eta \alpha} ;
$$

whence $\dot{\boldsymbol{a}}^{\prime}$ is determined.

This expression shows that the twist velocity produced by an impulsive wrench on a given rigid body constrained to twist about a given screw, varies directly as the product of the virtual coefficient of the two screws and the intensity of the impulsive wrench, and inversely as the square of $u_{\alpha}$.

$\S 61$. The Kinetic Energy acquired by an Impulse can be easily found by $\S 59$; for, from the last equation,

$$
M \dot{a}^{\prime 2} u_{a}^{2}=\frac{e^{2}}{M} \frac{\eta^{\prime \prime 2}}{u_{a}^{2}} \varpi_{\eta a^{2}}{ }^{2}
$$

hence the kinetic energy produced by the action of an impulsive wrench on a body constrained to twist about a given screw varies directly as the product of the square of the virtual coefficient of the two screws and the square of the intensity of the impulsive wrench, and inversely as the square of $u_{\alpha}$.

$\S 62$. Free Body.-We shall now express the kinetic energy communicated by the impulsive wrench on $\eta$ to the body when perfectly free. The component on $\omega_{m}$ of intensity $\eta^{\prime \prime} \eta_{m}$ imparts a kinetic energy equal to

$$
\frac{e^{2}}{M} \eta^{\prime \prime 2} \eta_{m}^{2}
$$


whence the total kinetic energy is found by adding these six terms.

The difference between the kinetic energy acquired when the body is perfectly free, and when the body is constrained to twist about $a$, is equal to

$$
\frac{e^{2}}{M} \frac{\eta^{\prime / 2}}{u_{\alpha}^{2}}\left[u_{\alpha}^{2}\left(\Sigma(\eta)^{2}\right)-\left(\Sigma\left(p_{1} a_{1} \eta_{1}\right)\right)^{2}\right] .
$$

The quantity inside the bracket reduces to the sum of 15 square terms, of which $\left(p_{1} a_{1} \eta_{2}-p_{2} a_{2} \eta_{1}\right)^{2}$ is a specimen. The entire expression being therefore essentially positive shows that a given impulse imparts greater energy to a quiescent body when free than to the same quiescent body when constrained to twist about a certain screw.

$\S 63$. Lemma.-If a group of instantaneous screws belong to a complex of the $n^{\text {th }}$ order, then the corresponding group of impulsive screws also belong to a complex of the $n^{\text {th }}$ order; for, suppose that $n+1$ twisting motions about $n+$ I screws neutralise, then the corresponding $n+\mathrm{I}$ impulsive wrenches must equilibrate, but this would not be possible unless all the impulsive screws belonged to a screw complex of the $n^{\text {th }}$ order.

$\S 64$. Euler's Theorem.-If a free or constrained rigid body receives an impulsive wrench, the body will commence to move with a larger kinetic energy when it is permitted to select its own instantaneous screw from the screw complex $P$ defining the freedom, than it would have acquired, had it been arbitrarily restricted to any other screw of the complex.

Let $Q$ be the reciprocal complex of the $\left(6-n^{t h}\right)$ order, and let $P^{\prime}$ be the screw complex of the $n^{\text {th }}$ order, consisting of those impulsive screws which, if the body were free, would correspond to the screws of $P$ as instantaneous screws. 
Let $\eta$ be any screw on which the body receives an impulsive wrench. Decompose this wrench into components on a system of six screws consisting of any $n$ screws from $P^{\prime}$, and any $6-n$ screws from $Q$. The latter are neutralised by the reactions of the constraints, and may be omitted, while the former compound into one wrench on a screw $\zeta$ belonging to $P^{\prime}$; we may therefore replace the given wrench by a wrench on $\zeta$. Now, if the body were perfectly free, an impulsive wrench on $\zeta$ must make the body twist about some screw $a$ on $P$. In the present case, although the body is not perfectly free, yet it is free so far as twisting about $\boldsymbol{a}$ is concerned, and we may therefore, with reference to this particular impulse about $\zeta$, consider the body as being perfectly free. It follows from $\S 62$ that there would be a loss of energy if the body were compelled to twist about any other screw than $a$, which is the one it naturally chooses. This theorem is due to Euler.*

$\S 65$. Co-ordinates of a Screw belonging to a Screw complex.-It will now be necessary to make some extensions of the conceptions of screw co-ordinates. Suppose that a body have freedom of the $n^{\text {th }}$ order, we have shown that it is always possible to choose $n$ screws from the screw complex expressing that freedom, such that each screw is reciprocal to all the rest. As an example we shall give the proof for the screw complex of the third order. Let $B_{1}, B_{2}, B_{3}$ be three screws of the reciprocal screw complex; then, if any screw $A_{1}$ be taken which is reciprocal to $B_{1}, B_{2}, B_{3}$, any screw $A_{2}$ which is reciprocal to $B_{1}, B_{2}, B_{3}, A_{1}$, and the screw $A_{3}$, which is reciprocal to $B_{1}, B_{2}, B_{3}, A_{1}, A_{2}$; then the group $A_{1}, A_{2}, A_{3}$ possess the required property, and may be termed co-reciprocals.

* Thomson \& Tait : Natural Philosophy, vol. i. p. 2 I6. 
The co-ordinates of a screw belonging to a given screw complex are simplified by taking $n$ co-reciprocal screws belonging to the given screw complex as a portion of the six screws of reference. In this case, out of the six co ordinates $a_{1}, \ldots a_{6}$ of a screw $a$, which belongs to the complex, $6-n$ are actually zero. Thus we are enabled to give a more general definition of screw coordinates, which will apply to a screw-complex of every order from I to 6 , both inclusive.

If a wrench, of which the intensity is one unit on a screw a, which belongs to a certain screw complex of the $n^{\text {th }}$ order, be decomposed into $n$ wrenches of intensities $a_{1}, \ldots a_{n}$ on $n$ co-reciprocal screws belonging to the same screw complex, then the $n$ quantities $a_{1}, \ldots a_{n}$ are said to be the co-ordinates of the screw a. Thus the pitch of $\alpha$ will be represented by $p_{1} a_{1}{ }^{2}+\ldots+p_{n} a_{n}{ }^{2}$. The virtual coefficient of $a$ and $\beta$ will be $2\left(p_{1} a_{1} \beta_{1}+\ldots+p_{n} a_{n} \beta_{n}\right)$.

We may here remark that one screw can always be found upon a screw complex of the $n^{\text {th }}$ order reciprocal to $n-1$ screws of the same complex. For, take $6-n$ screws of the reciprocal screw complex, then the required screw is reciprocal to $6-n+n-I=5$ known screws, and is therefore determined $(\S 27)$.

$\S 66$. The Reduced Wrench.-A wrench which acts upon a constrained rigid body may always be replaced by a wrench on a screw belonging to the screw complex, which defines the freedom of the body.

Take $n$ screws from the screw complex of the $n^{\text {th }}$ order which defines the freedom, and $6-n$ screws from the reciprocal complex. Decompose the given wrench into components on these six screws. The component wrenches on the reciprocal complex are neutralized by the reactions of the constraints, and may be discarded, while the remainder must compound into a wrench on the given screw complex. 
Whenever a given external wrench is replaced by an equivalent wrench upon a screw of the complex which defines the freedom of the body, the latter may be termed, for convenience, the reduced wrench.

It will be observed, that although the reduced wrench can always be determined from the given wrench, that the converse problem is indeterminate $(n<6)$.

We may state this result in a somewhat different manner. A given wrench can always be resolved into two wrenches-one on a screw of any given complex, and the other on a screw of the reciprocal screw complex. The former of these is what we denote by the reduced wrench.

$\S 67$. Co-ordinates of Impulsive and Instantaneous Screws.-Taking as screws of reference the $n$ principal screws of inertia ( $(57)$, we require to ascertain the relation between the co-ordinates of a reduced impulsive wrench and the co-ordinates of the corresponding instantaneous screw. If the co-ordinates of the reduced wrench are $\eta_{1}{ }^{\prime \prime}, \ldots, \eta_{n}{ }^{\prime \prime}$, and those of the corresponding twisting motion are $\dot{\boldsymbol{a}}_{1}{ }^{\prime}, \ldots, \dot{\boldsymbol{a}}_{n}{ }^{\prime}$, then, remembering the property of a principal screw of inertia ( $\$ 57$ ), and denoting by $u_{1}, \ldots, u_{n}$, the values of the magnitude $u(\S 59)$ for the principal screws of inertia, we have, from $\S 60$,

$$
\dot{a}_{1}^{\prime} \frac{u_{1}^{2}}{p_{1}}=\frac{e}{M} \eta_{1}^{\prime \prime}
$$

whence we deduce the following theorem, which, in the particular case of $n=6$, reduces to that of $\S 53$.

If a quiescent rigid body, which has freedom of the $n^{\text {th }}$ order, commence to twist about a screw $a$, of which the co-ordinates, with respect to the principal screws of inertia, are $\alpha_{1}, \ldots a_{n}$ and if $p_{1}, \ldots, p_{n}$ be the pitches, and $u_{1}, \ldots, u_{n}$ the constants defined, in $\S 59$, of the 
principal screws of inertia, then the co-ordinates of the reduced impulsive wrench are proportional to

$$
\frac{u_{1}^{2}}{p_{1}} \boldsymbol{a}_{1}, \ldots, \frac{u_{n}^{2}}{p_{n}} \boldsymbol{a}_{n} .
$$

Let $T$ denote the kinetic energy of the body of mass $M$ when animated by a twisting motion about the screw $a$, with a twist velocity $a^{\prime}$. Let the twist velocities of the components on any $n$ conjugate screws of inertia be denoted by $\dot{\boldsymbol{a}}_{1}{ }^{\prime}, \ldots \dot{\boldsymbol{a}}_{n}{ }^{\prime}$. (These screws will not be co-reciprocal unless in the special case where they are the principal screws of inertia.) It follows $(\$ 58)$ that the kinetic energy will be the sum of the $n$ several kinetic energies due to each component twisting motion. Hence we have $(\S 59)$

$$
T=M u u_{1}{ }^{2} \dot{a}_{1}{ }^{2}+\ldots+M u_{n}{ }^{2} \dot{a}_{n}{ }^{2},
$$

and also

$$
u_{a}^{2}=u_{1}^{2} a_{1}^{2}+\ldots+u_{n}^{2} a_{n}^{2} .
$$

Let $a_{1}, \ldots a_{n}$ and $\beta_{1}, \ldots, \beta_{n}$ be the co-ordinates of any two screws belonging to a screw complex of the $n^{\text {th }}$ order, referred to any $n$ conjugate screws of inertia, whether co-reciprocal or not, belonging to the same screw complex, then the condition that $a$ and $\beta$ should be conjugate screws of inertia is

$$
u_{1}^{2} a_{1} \beta_{1}+\ldots .+u_{n}^{2} a_{n} \beta_{n}=0 .
$$

To prove this, take the case of $n=4$, and let $A, B, C, D$ be the four screws of reference, and let $A_{1}, \ldots, A_{6}$ be the co-ordinates of $A$ with respect to the six principal screws of inertia of the body when free $\left(\S 5^{2}\right)$. The unit wrench on $\boldsymbol{a}$ is to be resolved into four wrenches of intensities $\boldsymbol{a}_{1}, \ldots, \boldsymbol{a}_{4}$ on $A, B, C, D$ : each of these compo nents is again to be resolved into six wrenches on the 
screws of reference. The six co-ordinates of $\boldsymbol{a}$, with respect to the same screws, are therefore

$$
\begin{gathered}
A_{1} a_{1}+B_{1} a^{z}+C_{1} a_{3}+D_{1} a_{4}, \\
\& c . \\
A_{6} a_{1}+B_{6} a_{2}+C_{6} a_{3}+D_{6} a_{4} .
\end{gathered}
$$

We can now express the condition that $a$ and $\beta$ are conjugate screws of inertia. This condition is ( $\$ 54)$

$$
\begin{aligned}
\Sigma p_{12}\left(A_{1} a_{1}+B_{1} a_{2}+C_{1} a_{3}+D_{1} a_{4}\right)\left(A_{1} \beta_{1}+B_{1} \beta_{2}\right. & +C_{1} \beta_{3} \\
& \left.+D_{1} \beta_{4}\right)=0 .
\end{aligned}
$$

Denoting $p_{1}^{2} A_{1}^{2}+\ldots+p_{6} A_{6}{ }^{2}$ by $u_{1}^{2}$, and observing that $\Sigma p_{1}^{2} A_{1} B_{1}$ and similar expressions are zero, we deduce

$$
u_{1}^{2} a_{1} \beta_{1}+\ldots+u_{4}^{2} a_{4} \beta_{4}=0 .
$$




\section{CHAPTER VII.}

THE POTENTIAL ENERGY OF A DISPLACEMENT.

$\S 68$. The Potential Energy of a Displacement.-Suppose a rigid body which possesses freedom of the $n^{\text {th }}$ order be submitted to the influence of any system of forces included within the restriction of $\S 6$. Let the symbol $O$ define a position of the body from which the forces would be unable to disturb it. By a twist of amplitude $\theta^{\prime}$ about a screw $\theta$ belonging to the screw complex of the $n^{\text {th }}$ order, which expresses the nature of the freedom, the body may be displaced from $O$ to an adjacent position $P$, while the energy consumed in making the twist is denoted by $V$. It appears from $\S 7$ that the same amount of energy would be required, whatever be the route by which the movement is made from $O$ to $P$. It follows that $V$ can only depend on certain constants and on the position of $P$ with respect to $O$. The most natural co-ordinates by which the position $P$ can be specified with respect to $O$ are the co-ordinates of the twist ( $\$ 34$ ) by which the movement from $O$ to $P$ could be effected. In general these co-ordinates will be six in number; but if $n$ of the screws of reference be selected from the screw complex defining the freedom of the body, then $(\$ 65)$ there will be only $n$ co-ordinates required, and these may be denoted by $\theta_{1}{ }^{\prime}, \ldots, \theta_{n}{ }^{\prime}$.

The Potential $V$ must therefore depend only upon certain constants relating to the forces and upon the $n$ quantities $\theta_{1}^{\prime}, \ldots, \theta_{n}^{\prime}$; and since these quantities are small, it follows that $V$ must be capable of development in a series of ascending powers and products of the co-ordinates, whence we may write 


$$
V=H+H_{1} \theta_{1}{ }^{\prime}+\ldots+H_{n} \theta_{n}{ }^{\prime}
$$

+ terms of the second and higher orders,

where $H, H_{1}, \ldots, H_{n}$ are constants, in so far as different displacements are concerned.

In the first place, it is manifest that $H=0$; because if no displacement be made, no energy is consumed. In the second place, $H_{1}, \ldots, H_{n}$ must also be each zero, because the position $O$ is one of equilibrium; and therefore, by the principle of virtual velocities, the work done by small twists about the screws of reference must be zero, as far as the first power of small quantities is concerned. Finally, neglecting all terms above the second order, on account of their minuteness, we see that the function $V$, which expresses the potential energy of a small displacement from a position of equilibrium, is a homogeneous function of the second degree of the $n$ co-ordinates, by which the displacement is defined:

$\S 69$. The Wrench evoked by Displacement.-When the body has been displaced to $P$, the forces no longer equilibrate, for a certain wrench has been evoked. We now propose to determine, by the aid of the function $V$, the co-ordinates of this wrench, or, more strictly, the coordinates of the equivalent reduced wrench ( $\$ 66)$ upon a screw of the complex, by which the freedom of the body is defined.

If, in making the displacement, work has been done by the agent which moved the body, then the equilibrium of the body was stable when in the position $O$, so far as this displacement was concerned. Let the displacement screw be 0 , and let a reduced wrench be evoked on a screw $\eta$ of the complex, while the intensities of the components on the screws of reference are $\eta_{1}{ }^{\prime \prime}, \ldots, \eta_{n}{ }^{\prime \prime}$. Suppose the body be displaced from $P$ to an excessively close position $P^{\prime}$, the co-ordinates of $P^{\prime}$, with respect to 
$O$, being $\theta_{1}{ }^{\prime}+\delta \theta_{1}{ }^{\prime}, \ldots \theta_{n}{ }^{\prime}+\delta \theta_{n}{ }^{\prime}(\S 65)$. The potential $V^{\prime}$ of the position $P^{\prime}$ is

$$
V+\frac{d V}{d \theta_{1}{ }^{\prime}} \delta \theta_{1}{ }^{\prime}+\ldots+\frac{d V}{d \theta_{n}{ }^{\prime}} \delta \theta_{n}{ }^{\prime},
$$

it being understood that $\delta \theta_{1}{ }^{\prime}, \ldots, \delta \theta_{n}{ }^{\prime}$ are infinitely small magnitudes of a higher order than $\theta_{1}^{\prime}, \ldots \theta_{n}^{\prime}$.

The work done in forcing the body to move from $P$ to $P^{\prime}$ is $V^{\prime}-V$. This must be equal to the work done in the twists about the screws of reference whose amplitudes are $\delta \theta_{1}{ }^{\prime}, \ldots, \delta \theta_{n}^{\prime}$, by the wrenches on the screws of reference whose intensities are $\eta_{1}{ }^{\prime \prime}, \ldots, \eta_{n}{ }^{\prime \prime}$. As the screws of reference are co-reciprocal, this work will be equal to ( $\S 35$ )

$$
-2 \eta_{1}^{\prime \prime} p_{1} \delta \theta_{1}^{\prime}+\ldots-2 \eta_{n}{ }^{\prime \prime} p_{n} \delta \theta_{n} .
$$

Since the expression just written must be equal to $V^{\prime}-V$ for every position $P^{\prime}$ in the immediate vicinity of $P$, we must have the coefficients of $\delta \theta_{1}{ }^{\prime}, \ldots, \delta \theta_{n}{ }^{\prime}$ equal in the two expressions, whence we have $n$ equations, of which the first is

$$
\eta_{1}^{\prime \prime}=-\frac{\mathbf{I}}{2 p_{1}} \frac{d V}{d \theta_{1}^{\prime}} .
$$

Hence, we deduce the following useful theorem:-

If a free or constrained rigid body be displaced from a position of equilibrium by twists of small amplitudes, $\theta_{1}{ }^{\prime}, \ldots, \theta_{n}{ }^{\prime}$, about $n$ co-reciprocal screws of reference, and if $V$ denote the work done in producing this movement, then the reduced wrench has, for components on the screws of reference, wrenches of which the intensities are found by dividing twice the pitch of the corresponding reference screw into the differential coefficient of $V$ 
with respect to the corresponding amplitude, and changing the sign of the quotient.

It is here interesting to notice that the co-ordinates of the reduced impulsive wrench referred to the principal screws of inertia, which would give the body a kinetic energy $T$ on the screw $\theta$, are proportional to

$$
\frac{\mathrm{I}}{2 p_{1}} \frac{d T}{d \dot{\theta}_{1}^{\prime}}, \ldots \frac{\mathrm{I}}{2 p_{n}} \frac{d T}{d \dot{\theta}_{n}^{\prime}}(\S 67) \text {. }
$$

$\S 70$. Conjugate Screws of the Potential.-Suppose that a twist about a screw $\theta$ evokes a wrench on a screw $\eta$, while a twist about a screw $\phi$ evokes a wrench on a screw $\zeta$. If $\theta$ be reciprocal to $\zeta$, then must $\phi$ be reciprocal to $\eta$. This will now be proved.

The condition that $\theta$ and $\zeta$ are reciprocal is

$$
p_{1} \theta_{1} \zeta_{1}+\ldots+p_{n} \theta_{n} \zeta_{n}=0
$$

but the intensities (or amplitudes) of the components of a wrench (or twist) are proportional to the co-ordinates of the screw on which the wrench (or twist) acts, whence the last equation may be written

$$
p_{1} \theta_{1}^{\prime} \zeta_{1}^{\prime \prime}+\ldots+p_{n} \theta_{n}^{\prime} \zeta_{n}^{\prime \prime}=0
$$

but we have seen $(\$ 69)$ that

$$
\zeta_{1}^{\prime \prime}=-\frac{1}{2 p_{1}} \frac{d V_{\phi}}{d \phi_{1}{ }^{\prime}}, \ldots \zeta_{n}^{\prime \prime}=-\frac{1}{2 p_{n}} \frac{d V_{\phi}}{d \phi_{n}{ }^{\prime}} ;
$$

whence the condition that $\theta$ and $\zeta$ are reciprocal is

$$
\theta_{1}{ }^{\prime} \frac{d V_{\phi}}{d \phi_{1}{ }^{\prime}}+\ldots+\theta_{n}{ }^{\prime} \frac{d V_{\phi}}{d \phi_{n}{ }^{\prime}}=0
$$

Now, as $V_{\phi}$ is an homogeneous function of the second order of the quantities $\phi_{1}{ }^{\prime}, \ldots, \phi_{n}{ }^{\prime}$, we may write 
POTENTIAL ENERGY OF A DISPLACEMENT.

$V \phi=A_{11} \phi_{1}^{\prime 2}+\ldots+A_{n n} \phi_{n}{ }^{\prime 2}+2 A_{13} \phi_{1}^{\prime} \phi_{2}^{\prime}+2 A_{23} \phi_{1}{ }^{\prime} \phi_{3}^{\prime}+\ldots$

in which $A_{h k}=A_{k h}$.

Hence we obtain-

$$
\frac{d V_{\phi}{ }^{\prime}}{d \phi_{1}{ }^{\prime}}=2\left\{A_{11} \phi_{1}{ }^{\prime}+A_{12} \phi_{2}{ }^{\prime}+\ldots .+A_{1 n} \phi_{n}{ }^{\prime} \cdot\right\}
$$

Introducing these expressions we find, for the condition that $\theta$ and $\zeta$ should be reciprocal.

$\theta_{1}{ }^{\prime}\left(A_{11} \phi_{1}{ }^{\prime}+\ldots A_{1 n} \phi_{n}{ }^{\prime}\right)+\ldots+\theta_{n}{ }^{\prime}\left(A_{n 1} \phi_{1}{ }^{\prime}+\ldots+A_{n n} \phi_{n}\right)=0$.

This may be written in the form :-

$$
A_{11} \theta_{1}^{\prime} \phi_{1}^{\prime}+\ldots, A_{n n} \theta_{n}^{\prime} \phi_{n}^{\prime}+A_{12}\left(\theta_{1}^{\prime} \phi_{2}^{\prime}+\theta_{2}^{\prime} \phi_{1}^{\prime}\right)+\ldots . .
$$

But this equation is perfectly symmetrical with respect to $\theta$ and $\phi$, and therefore we should have been led to the same result by expressing the condition that $\phi$ was reciprocal to $\eta$.

When $\theta$ and $\phi$ possess this property, they are said to be conjugate screws of the potential, and the condition that they should be so related, expressed in terms of their co-ordinates, is obtained by omitting the accents from the last equation.

If a screw $\phi$ be reciprocal to $\eta$, then $\phi$ is a conjugate screw of the potential to $\theta$. If we consider the screw $\theta$ to be given, we may regard the screw complex of the fifth order, which embraces all the iscrews reciprocal to $\eta$, as in a certain sense the locus of $\phi$. All the screws conjugate to $\theta$, and which, at the same time, belong to the screw complex $C$ by which the freedom of the body is defined, must constitute in themselves a screw complex of the $(n-1)^{\text {th }}$ order. For, besides fulfilling the $6-n$ conditions which define the screw complex $C$, they must also fulfil the condition of being reciprocal to $\eta$; but all 
the screws reciprocal to $7-n$ screws constitute a screw complex of the $(n-1)^{\text {th }}$ order $(\S 46)$.

The reader will be careful to observe the distinction between two conjugate screws of inertia (\$54), and two conjugate screws of the potential. Though these pairs possess some useful analogies, yet it should be borne in mind that the former are purely intrinsic to the rigid body, inasmuch as they only depend on the distribution of its material, while the latter involve extrinsic considerations, arising from the forces to which the body is submitted.

$\S 7$ I. Principal Screws of the Potential.-We are now going to prove that $n$ screws can be found such that when the body is displaced by a twist about any one of these screws, a reduced wrench is evoked about the same screw. The screws which possess this property are called the principal screws of the potential. . Let $a$ be a principal screw of the potential, then we must have, $\S 69$ :-

$$
\boldsymbol{a}_{1}^{\prime \prime}=-\frac{\mathrm{I}}{2 p_{1}} \cdot \frac{d V_{a}}{d \boldsymbol{a}_{1}^{\prime}},
$$

and $(n-I)$ similar equations.

Introducing the value of $V_{\alpha}$, and remembering ( $\left.\$ 36\right)$ that $a_{1}^{\prime \prime}=a^{\prime \prime} a_{1}$ and $a_{1}^{\prime}=a^{\prime} a_{1}$, we have the following $n$ equations :-

$$
\begin{gathered}
a_{1}\left(A_{11}+\frac{a^{\prime \prime}}{a^{\prime}} p_{1}\right)+a_{2} A_{12}+\ldots+a_{n} A_{1 n}=0, \\
\& c ., \& c . \\
a_{1} A_{n 1}+a_{2} A_{n 2}+\ldots+a_{n}\left(A_{n n}+\frac{a^{\prime \prime}}{a^{\prime}} p\right)=0
\end{gathered}
$$

From these linear equations $a_{1} \ldots, a_{n}$ can be eliminated, and we obtain an equation of the $n^{\text {th }}$ degree* in Art. 44 .

All the roots of this equation are real. See Salmon's Higher Algebra, 
$\frac{a^{\prime \prime}}{a^{\prime}}$. The values of $\frac{a^{\prime \prime}}{a^{\prime}}$ substituted successively in the linear equations just written will determine the co-ordinates of the $n$ principal screws of the potential.

We can now show that these $n$ screws are co-reciprocal. It is evident, in the first place, that if $S$ be a principal screw of the potential, and if $\theta$ be a displacement screw which evokes a wrench on $\eta$, the principle of $\S 70$ asserts that, when $\theta$ is reciprocal to $S$, then must also $\eta$ be reciprocal to $S$. Now, let the $n$ principal screws of the potential be denoted by $S_{1}, \ldots, S_{n}$, and let $T_{n}$ be that screw of the screw complex which is reciprocal to $S_{1}, \ldots, S_{n-1}(\S 65)$, then if the body be displaced by a twist about $T_{n}$, the wrench evoked must be on a screw reciprocal to $S_{1}, \ldots, S_{n-1}$; but $T_{n}$ is the only screw of the screw complex possessing this property; therefore a twist about $T_{n}$ must evoke a wrench on $T_{n}$, and therefore $T_{n}$ must be a principal screw of the potential. But there are only $n$ principal screws of the potential, therefore $T_{n}$ must coincide with $S_{n}$, and therefore $S_{n}$ must be reciprocal to $S_{1}, \ldots S_{n}$-1

\$2. Co-ordinates of the Wrench evoked by a Twist.The work done in giving the body a twist of small amplitude $\boldsymbol{a}^{\prime}$ about a screw $\boldsymbol{a}$, may be denoted by-

$$
F v_{a}^{2} a^{\prime 2}
$$

In fact, remembering that $a^{\prime} a_{1}=a_{1}^{\prime}, \ldots$, and substituting these values for $\boldsymbol{a}_{1}^{\prime}$ in $V(\$ 70)$, we deduce the expression :-

$$
F v_{a}^{2}=A_{11} a_{1}^{2}+\ldots+A_{n n} a_{n}^{2}+2 A_{12} a_{1} a_{2}+2 A_{13} a_{1} a_{3}+\ldots
$$

where $F$ is a certain constant, whose dimensions are a mass divided by the square of a time, and where $v_{a}$ is a linear magnitude specially appropriate to each screw $a$, and 
depending upon the co-ordinates of $a$, and the constants in the function $V(\S 68)$.

The parameter $v_{\alpha}$ may be constrasted with the parameter $u_{\alpha}$ considered in $\S 59$. Each is a linear magnitude, but the latter depends only upon the co-ordinates of $a$, and the distribution of the material of the rigid body. Both quantities may be contrasted with the pitch $p_{\alpha}$, which is also a linear magnitude, but depends solely on the screw, and is therefore purely geometrical.

If a body receive a twist of small amplitude $a^{\prime}$ about one of the principal screws of the potential, then the intensity of the wrench evoked on the same screw is $(\$ 69)$ :-

$$
-\frac{\mathrm{I}}{2 p_{\alpha}} \cdot \frac{d V_{\alpha}}{d \boldsymbol{\alpha}^{\prime}}
$$

but we have just seen that $V=F v_{a}^{\prime}{ }^{2} a^{\prime 2}$, whence we have the following theorem :-

If a body which has freedom of the $n^{\text {th }}$ order be displaced from a position of equilibrium by a twist about a screw $\alpha$, of which the co-ordinates with respect to the principal screws of the potential are $\boldsymbol{a}_{1}, \ldots, \boldsymbol{a}_{n}$, then a wrench $(\S 66)$ is evoked on a screw of which the co-ordinates are proportional to $\frac{v_{1}{ }^{2}}{p_{1}} a_{1}, \ldots, \frac{v_{n}{ }^{2}}{p_{n}} a_{n}$, where $v_{1}, \&$ c., $p_{1}, \&$ c., are the values of the quantity $v$, and the pitch $p$, for the principal screws of the potential.

We can now express with great simplicity the condition that two screws $\theta$ and $\phi$ shall be conjugate screws of the potential. For, if $\theta$ be reciprocal to the screw whose co-ordinates are proportional to-

we have :-

$$
\frac{v_{1}^{2}}{p_{l}} \phi_{1}, \ldots \frac{v_{n}^{2}}{p_{n}} \dot{\phi}_{n}
$$

$$
v_{1}^{2} \theta_{1} \phi_{1}+\ldots+v_{n}^{2} \theta_{n} \phi_{n}=0
$$


The expression for the potential assumes the simple form-

$$
F v_{1}^{2} a_{1}^{\prime 2}+\ldots+F v_{n}^{2} a_{n}^{\prime 2} .
$$

If the function $V$ be proportional to the product of the pitch of the displacement screw and the square of the amplitude, then every displacement screw will coincide with the screw about which the wrench is evoked.

$\S 73$. Form of the Potential.-The $n$ principal screws of the potential form a unique group, inasmuch as they are co-reciprocal, as well as being conjugate screws of the potential. They therefore fulfil $15+15=30$ conditions, being the total number available in the selection of six screws.

We are now going to show that the expression of the potential will consist of the sum of $n$ square terms, whenever it is referred to any set of $n$ conjugate screws of the potential.

The energy consumed in giving a body a twist of amplitude $\theta$ from the position of equilibrum $O$ to a new position $P$, is equal to $F v_{\theta}^{2} \theta^{\prime 2}(\S 72)$, and $\eta$ is the screw on which the wrench is evoked. Suppose that now from the position $P$ the body receive a twist of amplitude $\phi^{\prime}$ about a screw $\phi$, it would generally not be correct to assert that the energy consumed in the second twist was proportional to the square of its amplitude. For, during the second twist, either a portion of the energy will be consumed in doing work against the wrench on $\eta$, or the energy expended in the second twist will be rendered less, in consequence of the assistance afforded by the wrench on $\eta$. If, however, $\eta$ be reciprocal to $\phi$, then the quantity of energy consumed in the twist about $\phi$ will be unaffected by the presence of a wrench on $\eta$. Hence if $\theta$ and $\phi$ be two conjugate screws of the potential, 
the energy expended in giving the body first a twist of amplitude $\theta^{\prime}$ about $\theta$, and then a twist of amplitude $\phi^{\prime}$ about $\phi$, is to be represented by-

$$
F v_{\theta}^{2} \theta^{\prime 2}+F v_{\phi}^{2} \phi^{\prime 2} .
$$

By taking a third screw, conjugate to both $\theta$ and $\phi$, and then a fourth screw conjugate to the remaining three, and so on, we see finally that the potential reduces to the sum of $n$ square terms, when each pair of the screws of reference are conjugate screws of the potential. 


\section{CHAPTER VIII.}

\section{HARMONIC SCREWS.}

$\S 74$. Definition of an Harmonic Screw.-We have seen in $\S 67$ that to each screw $\theta$ of a screw complex of the $n^{\text {th }}$ order corresponds a screw $\lambda$, belonging to the same screw complex. The relation between $\theta$ and $\lambda$ is determined when the rigid body, and also the screw complex which defines its freedom, are completely known. The physical connexion between the two screws $\theta$ and $\lambda$ may be thus stated. If a wrench act on the screw $\lambda$ for a short time, the body, if previously quiescent, will commence to move by twisting about $\theta(\$ 67)$.

We have also seen $(\$ 72)$ that to each screw $\theta$ of a screw complex of the $n^{\text {th }}$ order corresponds a certain screw $\boldsymbol{\eta}$ belonging to the same screw complex. The relation between $\theta$ and $\eta$ is determined when the rigid body, the forces, and the screw complex which defines the freedom, is known. The physical connexion between the two screws $\theta$ and $\eta$ may be thus stated. If the body be displaced from a position of equilibrium by a twist about $\theta$, the evoked wrench, when reduced $(\$ 66)$ to the screw complex, acts on $\eta$.

The rigid body being given in a position of equilibrium, and the forces which act on the body being known, and also the screw complex by which the freedom of the body is prescribed, we then have corresponding to each screw $\theta$ of the given screw complex, two other screws $\lambda$ and $\eta$, which also belong to the same screw complex.

Considering the very different physical character of 
the two systems of correspondence, it will of course usually happen that the two screws $\lambda$ and $\eta$ are not identical. But a little reflection will enable us to foresee what we shall afterwards prove, viz., that when $\theta$ has been appropriately chosen, then $\lambda$ and $\eta$ may coincide. For since $n-\mathrm{I}$ arbitrary quantities are disposable in the selection of a screw from a screw complex of the $n^{\text {th }}$ order ( $\left.\$ 43\right)$, it follows that for any two screws (for example $\lambda$ and $\eta$ ) to coincide, $n-I$ conditions must be fulfilled; but this is precisely the number of arbitrary elements available in the selection of $\theta$. We can thus conceive that for one or more particular screws $\theta$, the two corresponding screws $\lambda$ and $\eta$ are identical; and we shall now prove the following important theorem :-

If a rigid body be displaced from a position of equilibrium by a twist about a screw $\theta$, and if the evoked wrench tend to make the body commence to twist about the same screw $\theta$, then $\theta$ may be called an harmonic screw, and the number of harmonic screws is the same as the order of the screw complex which defines the freedom of the rigid body.

We shall adopt as the screws of reference the $n$ principal screws of inertia. The impulsive screw, which corresponds to $\theta$ as an instantaneous screw, will have for co-ordinates-

$$
h \frac{u_{1}^{2}}{p_{1}} \theta_{1}, \ldots, h \frac{u_{u_{n}^{2}}^{2}}{p_{n}} \theta_{n}(\S 67),
$$

where $h$ is a certain constant which is determined by making the co-ordinates satisfy the condition ( $\$ 37$ ). If $\theta$ be a harmonic screw, then, remembering that the screws of reference are co-reciprocal ( $\$ 57$ ), we must have $n$ equations, of which the first is $(\S 72)$ :-

$$
h \frac{u_{1}^{2}}{\dot{p}_{1}} \theta_{1}^{\prime \prime}=-\frac{\mathbf{I}}{2 \dot{p}_{1}} \cdot \frac{d V}{d \theta_{1}^{\prime}} .
$$


Assuming $\frac{h \theta^{\prime \prime}}{\theta^{\prime}}=-M s^{2}$, where $M$ is the mass of the body, and $s$ an unknown quantity, and substituting for $V$ its value, we deduce the $n$ equations :-

$$
\begin{gathered}
\theta_{1}\left(A_{11}-M s^{2} u_{1}^{2}\right)+\theta_{2} A_{12}+\ldots+\theta_{n} A_{1 n}=0, \\
\& c ., \quad \& c . \\
\theta_{1} A_{n 1}+\theta_{2} A_{n 3}+\ldots+\theta_{n}\left(A_{n n}-M s^{2} u_{n}^{2}\right)=0 .
\end{gathered}
$$

Eliminating $\theta_{1}, \ldots, \theta_{n}$, we have an equation of the $n^{\text {th }}$ degree for $s^{2}$. The $n$ roots of this equation are all real, and each one substituted in the set of $n$ equations will determine, by a system of $n$ linear equations, the ratios of the $n$ co-ordinates of one of the harmonic screws.

It is a remarkable property of the $n$ harmonic screws that each pair of them are conjugate screws of inertia, and also conjugate screws of the potential. Let $H_{1}, \ldots$, $H_{n-1}$, be $n-1$ of the harmonic screws, to which correspond the impulsive screws $S_{1}, \ldots, S_{n-1}$. Also suppose $T$ to be that one screw of the given screw complex which is reciprocal to $S_{1}, \ldots, S_{n-1}(\S 65)$, then $T$ must form with each one of the screws $H_{1}, \ldots, H_{n-1}$ a pair of conjugate screws of inertia ( $\$ 54)$. Also, since $S_{1}, \ldots$, $S_{n-1}$ are the screws on which wrenches are evoked by twists about $H_{1}, \ldots, H_{n-1}$ respectively, it is evident that $T$ must form with each one of these screws $H_{1}, \ldots$, $H_{n-1}$ a pair of conjugate screws of the potential (\$70). It follows that the impulsive screw, corresponding to $T$ as the instantaneous screw, must be reciprocal to $H_{1}, \ldots$. $H_{n-1}$; and also that a twist about $T$ must evoke a wrench on a screw reciprocal to $H_{1}, \ldots H_{n-1}$. But as we can only have one screw of the screw complex reciprocal to $H_{1}, \ldots H_{n-1}$, it follows that the impulsive screw, which corresponds to $T$ as an instantaneous screw, must also be the screw on which a wrench is evoked by a twist about 
$T$. Hence, $T$ must be a harmonic screw, and as there are only $n$ harmonic screws, it is plain that $T$ must coincide with $H_{n}$, and that therefore $H_{n}$ is a conjugate screw of inertia, as well as a conjugate screw of the potential, to each one of the remaining $n-1$ harmonic screws. Similar reasoning will, of course, apply to each of the harmonic screws taken in succession.

$\S 75$. Equations of Motion.-We now consider the kinetical problem, which may be thus stated. A free or constrained rigid body, which is acted upon by a system. of forces, is displaced by an initial twist of small amplitude, from a position of equilibrium. The body also receives an initial twisting motion, with a small twist velocity, and is then abandoned to the influence of the forces. It is required to ascertain the nature of its subsequent movements.

Let $T$ represent the kinetic energy of the body, in the position of which the co-ordinates, referred to the principal screwes of inertia, are $\theta_{1}^{\prime}, \ldots, \theta_{n}{ }^{\prime}$. Then we have $(\$ 67):-$

$$
T=M\left[u_{1}^{2}\left(\frac{d \theta_{1}^{\prime}}{d t}\right)^{2}+\ldots+u_{n}^{2}\left(\frac{d \theta_{n}^{\prime}}{d t}\right)^{2}\right]
$$

while the potential energy which, as before, we denote by $V$, is an homogeneous function of the second order of the quantities $\theta_{1}^{\prime}, \ldots, \theta_{n}^{\prime}$.

By the use of Lagrange's method of generalized coordinates* we are enabled to write down at once the $n$ equations of motion in the form :-

$$
\frac{d}{d t}\left(\frac{d T}{d \frac{d \theta_{1}^{\prime}}{d t}}\right)+\frac{d V}{d \theta_{1}^{\prime}}=0 \text {. }
$$

* Mécanique Analytique, vol. i., p. 304. See also Thomson and Tait's Natural Philosophy, vol. i., p. 253. 
Substituting for $T$ we have :-

$$
2 M u_{1}^{2} \frac{d^{2} \theta_{1}^{\prime}}{d t^{2}}+\frac{d V}{d \theta^{\prime}}=0,
$$

with $(n-1)$ similar equations. Finally, introducing the expression for $V(\S 70)$, we obtain $n$ linear differential equations of the second order.

The reader who is not acquainted with Lagrange's magnificent equations of motion in generalized co-ordinates will perhaps welcome reasoning by which the equations which we require can be otherwise demonstrated.

Suppose the body to be in motion under the influence of the forces, and that at any epoch $t$ the co-ordinates of the twisting motion are $\frac{d \theta_{1}^{\prime}}{d t^{\prime}}, \ldots \frac{d \theta_{n}^{\prime}}{d t}$, when referred to the principal screws of inertia. Let $\boldsymbol{\zeta}_{1}{ }^{\prime \prime}, \ldots, \boldsymbol{\zeta}_{n}{ }^{\prime \prime}$ be the co-ordinates of a wrench which, had it acted upon the body at rest for the small time $e$, would have communicated to the body a twisting motion identical with that which the body actually has at the epoch $t$. The co-ordinates of the impulsive wrench which would, in the time $e$, have produced from rest the motion which the body actually has at the epoch $t+e$, are :-

$$
\zeta_{1}^{\prime \prime}+e \frac{d \zeta_{1}^{\prime \prime}}{d t}, \ldots, \zeta_{n}^{\prime \prime}+e \frac{d \zeta_{n}^{\prime \prime}}{d t},
$$

On the other hand, the motion at the epoch $t+e$ may be considered to arise from the influence of the wrench $\zeta_{1}^{\prime \prime}, \ldots . \zeta_{n}^{\prime \prime}$ for the time $e$, followed by the influence of the evoked wrench for the time $e$. The final effect of the two wrenches must, by the second law of motion, be the same as if they acted simultaneously for the time $e$ upon the body initially at rest.

The co-ordinates of the evoked wrench being:- 


$$
-\frac{\mathrm{I}}{2 p_{1}} \frac{d V}{d \theta_{1}^{\prime \prime}}, \ldots-\frac{\mathrm{I}}{2 p_{n}} \frac{d V}{d \theta_{n}^{\prime \prime}}
$$

we therefore have the equation:-

$$
\zeta_{1}^{\prime \prime}+e \frac{d \zeta_{1}^{\prime \prime}}{d t}=\zeta_{1}^{\prime \prime}-\frac{1}{2 p_{1}} \frac{d V}{d \theta_{1}},
$$

or-

$$
e \frac{d \zeta_{1}^{\prime \prime}}{d t}=-\frac{1}{2 p_{1}} \frac{d V}{d \theta_{1}^{\prime \prime}}
$$

and $n-1$ similar equations; but we see from $\S 67$ that-

$$
e_{\zeta_{1}^{\prime \prime}}^{\prime \prime}=M \frac{u_{1}^{2}}{p_{1}} \frac{d \theta_{1}^{\prime}}{d t} .
$$

Differentiating this equation with respect to the time, and regarding $e$ as constant, we have:-

$$
e \frac{d \zeta_{1}^{\prime \prime}}{d t}=M \frac{u_{1}^{2}}{p_{1}} \frac{d^{2} \theta_{!}^{\prime}}{d t^{2}}
$$

whence-

$$
2 M u_{1}^{2} \frac{d^{2} \theta_{1}^{\prime}}{d t^{2}}+\frac{d V}{d \theta_{1}^{\prime}}=0
$$

the same equation as that already found by Lagrange's method.

To integrate the equations we assume :-

$$
\theta_{1}^{\prime}=f_{1} \Omega, \ldots \theta_{n}^{\prime}=f_{n} \Omega ;
$$

where $f_{1}, \ldots f_{n}$ are certain constants, which will be determined, and where $\Omega$ is an unknown function of the time: introducing also the value of $V$, given in $\S 70$, we find for the equations of motion:-

$$
\begin{gathered}
M u_{1}^{2} f_{1} \frac{d^{2} \Omega}{d t^{2}}+\left(A_{11} f_{1}+A_{12} f_{2}+\ldots+A_{1 n} f_{n}\right) \Omega=0 \\
\quad \& c . \\
M u_{n}^{2} f_{n} \frac{d^{2} \Omega}{d t^{2}}+\left(A_{n 1} f_{1}+A_{n 2} f_{1}+\ldots+A_{n n} f_{n}\right) \Omega=0
\end{gathered}
$$


If the quantity $s$, and the ratios of the $n$ quantities $f_{1}, \ldots f_{n}$, be determined by the $n$ equations :-

$$
\begin{aligned}
& f_{1}\left(A_{11}-M u_{1}{ }^{2} s^{2}\right)+ f_{2} A_{12}+\ldots+f_{n} A_{n 1}=0, \\
& \& c ., \quad \& c . \\
& f_{1} A_{n 1}+f_{2} A_{2 n}+\ldots+f_{n}\left(A_{a n}-M u_{n}{ }^{2} s^{2}\right)=0 ;
\end{aligned}
$$

then the $n$ equations of motion will reduce to the single equation:-

$$
\frac{d^{2} \Omega}{d t^{2}}+s^{2} \Omega=0 .
$$

By eliminating $f_{1}, \ldots, f_{n}$ from the $n$ equations, we obtain precisely the same equation for $s^{2}$ as that which arose ( $(74)$ in the determination of the $n$ harmonic screws. The values of $f_{1}, \ldots, f_{n}$, which correspond to any value of $s^{2}$, are therefore proportional to the co-ordinates of a harmonic screw.

The equation for $\Omega$ gives :-

$$
\mathbf{\Omega}=H \sin (s t+c) \text {. }
$$

Let $H_{1}, \ldots H_{n}, c_{1}, \ldots c_{n}$ be $2 n$ arbitrary constants. Let $f_{p q}$ denote the value of $f_{q}$, when the root $s_{p}{ }^{2}$ has been substituted in the linear equations. Then by the known theory of linear differential equations,*

$$
\begin{aligned}
& \theta_{1}^{\prime}=f_{11} H_{1} \sin \left(s_{1} t+c_{1}\right)-\ldots+f_{n 1} H_{n} \sin \left(s_{n} t+c_{n}\right), \\
& \quad \& c ., \quad \& c . \\
& \theta_{n}^{\prime}=f_{1 n} H_{1} \sin \left(s_{1} t+c_{1}\right)+\ldots+f_{n n} H_{n} \sin \left(s_{n} t+c_{n}\right) .
\end{aligned}
$$

In proof of this solution it is sufficient to observe, that the values of $\theta_{1}^{\prime}, \ldots \theta_{n}^{\prime}$ satisfy the given differential equations of motion, while they also contain the requisite number of arbitrary constants.

* Lagrange's Mécanique Analytique, vol. i., p. 353. 
$\S 76$. Discussion of the Results.-For the position of the body before its displacement to have been one of stable equilibrium, it is manifest that the co-ordinates must not increase indefinitely with the time, and therefore all the values of $s^{2}$ must be essentially positive, since otherwise the values of $\theta_{1}^{\prime}, \ldots \theta_{n}^{\prime}$ would contain exponential terms.

The $2 n$ arbitrary constants are to be determined by the initial circumstances. The initial displacement is to be resolved into $n$ twists about the $n$ screws of reference ( $\S 65)$. This will provide $n$ equations, by making $t=0$, and substituting for $\theta_{1}^{\prime}, \ldots \theta_{n}^{\prime}$, in the equations just mentioned, the amplitudes of the initial twists. The initial twisting motion is also to be resolved into twisting motions about the $n$ screws of reference. The twist velocities of these components will be the values of $\frac{d \theta_{1}^{\prime}}{d t}, \&$ c., $\frac{d \theta_{n}^{\prime}}{d t}$, when $t=0$; whence we have $n$ more equations to complete the determination of the arbitrary constants.

If the initial circumstances be such that the constants $H_{2}, \ldots, H_{n}$ are all zero, then the equations assume a very simple form :-

$$
\begin{gathered}
\theta_{1}{ }^{\prime}=f_{11} H_{1} \sin \left(s_{1} t+c\right), \\
\& c . \\
\theta_{n}{ }^{\prime}=f_{1 n} H_{1} \sin \left(s_{1} t+c\right) .
\end{gathered}
$$

The interpretation of this result is very remarkable. We see that the co-ordinates of the body are always proportional to $f_{11}, \ldots, f_{1 n}$; hence the body can always be brought from the initial position to the position at any time by twisting it about that screw, whose co-ordinates are proportional to $f_{11}, \ldots, f_{1 n}$; but, as we have already pointed out, the screw thus defined 
is an harmonic screw, and hence we have the following theorem :-

If a rigid body occupy a position of stable equilibrium under the influence of a system of forces, as restricted in $\$ 6$, then $n$ harmonic screws can be selected from the screw complex of the $n^{\text {th }}$ order, which defines the freedom of the body, and if the body be displaced from its position of equilibrium by a twist about a harmonic screw, and if it also receive a small initial twist velocity about the same screw, then the body will continue for ever to perform twist oscillations about that harmonic screw, and the amplitude of the twist will be always equal to the arc of a certain circular pendulum, which has an appropriate length, and was appropriately started.

The integrals in their general form prove the following theorem :-

A rigid body is slightly displaced by a twist from a position of stable equilibrium under the influence of a system of forces, and the body receives a small initial twisting motion. The twist, and the twisting motion, may each be resolved into their components on the $n$ harmonic screws : $n$ circular pendulums are to be constructed, each of which is isochronous with one of the harmonic screws. All these pendulums are to be started at the same instant as the rigid body, each with an arc, and an angular velocity equal to the initial amplitude of the twist, and the twist velocity, which has been assigned to the corresponding harmonic screw, as its share of the initial circumstances. To ascertain where the body would be at any future epoch, it will only be necessary to calculate the arcs of the $n$ pendulums for that epoch, and then give the body twists from its position of equilibrium about the harmonic screws, whose amplitudes are equal to these arcs.

The reader will observe that the solution to which 
we have been conducted possesses the features which we have pointed out in $\S \mathbf{I} \mathbf{I}$, as characterising a complete discussion of a problem in the dynamics of a rigid body.

$\S 77$. Remarks on Harmonic Screws.-We may to a certain extent see the actual reason why the body, when once oscillating upon a harmonic screw, will never depart therefrom. The body, when displaced from the position of equilibrium by a twist upon a harmonic screw $\theta$, and then released, is acted upon by the wrench upon a certain screw $\eta$, which is evoked by the twist. But the actual effect of an impulsive wrench on $\boldsymbol{\eta}$ would be to make the body twist about the harmonic screw, and as the continued action of the wrench on $\eta$ is indistinguishable from an infinite succession of infinitely small impulses, we can find in the influence of the forces no cause adequate to change the motion of the body from twisting about the harmonic screw $\theta$. 


\section{$(83)$}

\section{CHAPTER IX.}

THE DYNAMICS OF A RIGID BODY, WHICH HAS FREEDOM OF THE FIRST ORDER.

$\S 78$. Introduction.-In the present chapter we shall apply the principles developed in the preceding chapters to the examination of the Dynamics of a rigid body which has freedom of the first order. The ensuing chapters will be similarly devoted to the other orders of freedom. We shall in each chapter first ascertain what can be learned as to the kinematics of a rigid body, so far as small displacements are concerned, from merely knowing the order of the freedom which is permitted by the constraints. This will conduct us to a knowledge of the screw complex which exactly defines the freedom enjoyed by the body. We shall then be enabled to determine the reciprocal screw complex, which involves the theory of equilibrium. The next group of questions will be those which relate to the effect of an impulse upon a quiescent rigid body, free to twist about all the screws of the screw complex. . Finally, we shall discuss the small oscillations of a rigid body in the vicinity of a position of stable equilibrium, under the influence of a given system of forces, the movements of the body being limited as before to the screws of the screw complex.

$\S 79$. Screw Complex of the First Order.-A body which has freedom of the first order can execute no movement which is not a twist about one definite screw. The position of a body so circumstanced is to be specified by a single datum, viz., the amplitude of the twist about the given screw, by which the body can be brought 
from a standard position to any other position which it is capable of attaining. As examples of a body which has freedom of the first order, we may refer to the case of a body free to rotate about a fixed axis, but not to slide along it, or of a body free to slide along a fixed axis, but not to rotate around it. In the former case the screw complex consists of one screw, whose pitch is zero; in the latter case the screw complex consists of one screw, whose pitch is infinite.

$\S 80$. The Reciprocal Screw Complex.-The integer which denotes the order of a screw complex, and the integer which denotes the order of the reciprocal screw complex, will, in all cases, have the number six for their sum $(\S 46)$. Hence a screw complex of the first order will have as its reciprocal a screw complex of the fifth order.

We shall, therefore, be obliged to discuss in the present chapter some properties of the screw complex of the fifth order, and so far to anticipate what would more naturally fall under Chapter XIII.

For a screw $\theta$ to belong to a screw complex of the fifth order, the necessary and sufficient condition is, that $\theta$ be reciprocal to one given screw $a$. This condition is thus expressed:-

$$
\left(p_{a}+p_{\theta}\right) \cos O-d \sin O=0,
$$

where $O$ is the angle, and $d$ the perpendicular distance between the screws $\theta$ and $a$.

We can now show that every straight line in space, when it receives an appropriate pitch, constitutes a screw of a given screw complex of the fifth order. For the straight line and $a$ being given, $d$ and $O$ are determined, and hence the pitch $p_{\theta}$ can be determined by the linear equation just written.

Consider now a point $A$, and the screw a. Every straight line through $A$, when furnished with the proper 
pitch, will be reciprocal to $a$. Since the number of lines through $A$ is doubly infinite, it follows that a singly infinite number of screws of given pitch can be drawn through $A$, so as to be reciprocal to $a$. We shall now prove that all the screws of the same pitch which pass through $A$, and are reciprocal to a, lie in a plane. This we shall first show to be the case for all the screws of zero pitch,* and then we 'shall deduce the more general theorem.

By a twist of small amplitude about $a$ the point $A$ is moved to an adjacent point $B$. To effect this movement against a force at $A$ which is perpendicular to $A B$, no work will be required; hence every line through $A$, perpendicular to $A B$, may be regarded as a screw of zero pitch, reciprocal to $a$.

We must now enunciate a principle which applies to a screw complex of any order. We have already referred to it with respect to the cylindroid ( $\$ 20)$. If all the screws of a screw complex be modified by the addition of the same linear magnitude (positive or negative) to the pitch of every screw, then the collection of screws thus modified still form a screw complex of the same order. The proof is obvious, for since the virtual co-efficient depends on the sum of the pitches, it follows that, if all the pitches of a complex be increased by a certain quantity, and all the pitches of the reciprocal complex be diminished by the same quantity, then all the first group of screws thus modified are reciprocal to all the second group as modified. Hence, since a screw

* This theorem is due to Möbius, who has shown, that, if small rotations about six axes can neutralise, and if five of the axes be given, and a point on the sixth axis, then the sixth axis is limited to a plane. (Ueber die Zusammensetzung unendlich kleiner Drehungen Crelle's Journal, t. xviii., pp. 189212). 
complex of the $n^{\text {th }}$ order consists of all the screws reciprocal to $6-n$ screws, it follows that the modified group must still be a screw complex.

We shall now apply this principle to prove that all the screws $\lambda$ of any given pitch $k$, which can be drawn through $A$, to be reciprocal to $\alpha$, lie in a plane. Take a screw $\eta$, of pitch $p_{a}+k$, on the same line as $a$, then we have just shown that all the screws $\mu$, of zero pitch, which can be drawn through the point $A$, so as to be reciprocal to $\eta$, lie in a plane. Since $\mu$ and $\eta$ are reciprocal, the screws on the same straight lines as $\mu$ and $\eta$ will be reciprocal, provided the sum of their pitches is the pitch of $\eta$; therefore, a screw $\lambda$, of pitch $k$, on the same straight line as $\mu$, will be reciprocal to the screw $a$, of pitch $p_{a}$; but all the lines $\mu$ lie in a plane, therefore all the screws $\lambda$ lie in the same plane.

Conversely, given a plane and a pitch $k$, a point $A$ can be determined in that plane, such that all the screws drawn through $A$ in the plane, and possessing the pitch $k$, are reciprocal to $a$. To each pitch $k_{1}, k_{2}, \ldots$, will correspond a point $A_{1}, A_{2} \ldots$; and it is worthy of remark, that all the points $A_{1}, A_{2}$ must lie on a right line which intersects $\alpha$ at right angles; for join $A_{1}$, $A_{2}$, then a screw on the line $A_{1} A_{2}$, which has for pitch either $k_{1}$ or $k_{2}$, must be reciprocal to $a$; but this is. impossible unless $A_{1} A_{2}$ intersect $\boldsymbol{a}$ at a right angle.

$\$ 81$. Equilibrium. - If a body which has freedom of the first order be in equilibrium, then the necessary and sufficient condition is, that the forces which act upon the body shall constitute a wrench on a screw of the screw complex of the fifth order, which is reciprocal to the screw which defines the freedom. We thus see that every straight line in space may be the residence of a screw, a wrench on which is consistent with the equilibrium of the body. 
If two wrenches act upon the body, then the condition of equilibrium is, that, when the two wrenches are compounded by the aid of the cylindroid, the single wrench which replaces them shall lie upon that one screw of the cylindroid, which is reciprocal to $a(\S 28)$.

We can express with great facility, by the aid of screw co-ordinates, the condition that wrenches of intensities $\theta^{\prime \prime}, \phi^{\prime \prime}$, on two screws $\theta, \phi$, shall equilibrate, when applied to a body only free to twist about $\boldsymbol{a}$.

Adopting any six co-reciprocals as screws of reference, and resolving each of the wrenches on $\theta$ and $\phi$ into its six components on the six screws of reference, we shall have for the intensity of the component of the resultant wrench on $\omega_{n}-$

$$
\theta^{\prime \prime} \theta_{n}+\phi^{\prime \prime} \phi_{n} \text {. }
$$

Hence the co-ordinates of the resultant wrench are proportional to-

$$
\theta^{\prime \prime} \theta_{1}+\phi^{\prime \prime} \phi_{1}, \ldots \theta^{\prime \prime} \theta_{6}+\phi^{\prime \prime} \phi_{6} .
$$

For equilibrium this screw must be reciprocal to $\alpha$, whence we have-

$$
p_{1} a_{1}\left(\theta^{\prime} \theta_{1}+\phi^{\prime \prime} \phi_{1}\right)+\& c .+p_{6} a_{6}\left(\theta^{\prime \prime} \theta_{6}+\phi^{\prime \prime} \phi_{6}\right)=0,
$$

or,

$$
\theta^{\prime \prime} \varpi_{a \theta}+\phi^{\prime \prime} \varpi_{a \phi}=0 .
$$

This equation merely expresses that the sum of the works done in a small twist about $\boldsymbol{a}$ against the wrenches on $\theta$ and $\phi$ is zero.

We also perceive that a given wrench may be always replaced by a wrench of appropriate intensity on any other screw, in so far as the effect on a body only free to twist about $\boldsymbol{a}$ is concerned.

It may not be out of place to notice the analogy which the equation just written bears to the simple prob- 
lem of the determination of the condition that two forces should be unable to disturb the equilibrium of a particle only free to move on a straight line. If $P, Q$ be the two forces, and if $l, m$ be the angles which the forces make with the direction in which the particle can move, then the condition is-

$$
P \cos l+Q \cos m=0 \text {. }
$$

This suggests what it will be well for the reader constantly to bear in mind, and that is, the analogy which subsists between the virtual co-efficient of two screws, and the cosine of the angle between two lines.

§ 82. Particular Case.-If a body having freedom of the first order be in equilibrium under the action of gravity, then the vertical through the centre of inertia must lie in the plane of reciprocal screws of zero pitch, drawn through the centre of inertia.

\$ 83. Impulsive Forces.-If a wrench of great intensity $\eta^{\prime \prime}$ act for a short time on the screw $\eta$, while the body is only permitted to twist about $a$, then we have seen in $\S 60$ how the twist velocity produced can be found. We shall now determine the impulsive reaction of the constraints. This reaction is a wrench of intensity $\lambda^{\prime \prime}$ on a screw $\lambda$, which is reciprocal to $a$. The determination of $\lambda$ may be effected geometrically in the following manner :-Let $\mu$ be the screw, an impulsive wrench on which would, if the body were perfectly free, cause an instantaneous twisting motion about $\boldsymbol{a}(\S 53)$. Draw the cylindroid $(\eta, \mu)$. Then $\lambda$ must be that screw on the cylindroid which is reciprocal to $a$, for a wrench on $\lambda$, and the given wrench on $\eta$, must compound into a wrench on $\mu$, whence the three screws must be co-cylindroidal ;*

* We shall often for convenience speak of three screws on the same cylindroid as co-cylindroidal. 
also $\lambda$ must be reciprocal to $a$, so that its position on the cylindroid is known ( $\$ 28)$. Finally, as the intensity $\eta^{\prime \prime}$ is given, and as the three screws $\eta, \lambda, \mu$ are all known, the intensity $\lambda^{\prime \prime}$ becomes determined ( $(17)$.

$\S 84$. Small Oscillations.-We shall now suppose that a rigid body which has freedom of the first order occupies a position of stable equilibrium under the influence of a system of forces, as restricted in $\S 6$. If the body be displaced by a small twist about the screw $a$ which prescribes the freedom, and if it further receive a small initial twist velocity about the same screw, the body will continue for ever to perform small twist oscillations about the screw $a$. We propose to determine the time of one oscillation.

The kinetic energy of the body, when animated by a twist velocity $\frac{d a^{\prime}}{d t}$ is $M u_{a}^{2}\left(\frac{d a^{\prime}}{d \iota^{\prime}}\right)^{2}(\S 59)$. The potential energy due to the position attained by giving the body a twist of amplitude $\boldsymbol{a}^{\prime}$ away from its position of equilibrium, is $F v_{\alpha}^{2} a^{\prime 2}(\$ 72)$. But the sum of the potential and kinetic energies must be constant ( $\$ 6)$, whence-

$$
M u_{a}^{2}\left(\frac{d a^{\prime}}{d t}\right)^{2}+F v_{a}^{2} a^{\prime 2}=\text { const. }
$$

Differentiating we have-

$$
\frac{d^{2} a^{\prime}}{d t^{2}}+\frac{F v_{a}^{2}}{M u_{a}^{2}} a^{\prime}=0 .
$$

Integrating this equation we have-

$$
a^{\prime}=A \sin \sqrt{\frac{F v_{\alpha}^{2}}{M u_{\alpha}{ }^{2}}} t+B \cos \sqrt{\frac{F v_{\alpha}^{2}}{M u_{\alpha}^{2}}} t
$$

where $A$ and $B$ are arbitrary constants. The time of one oscillation is therefore- 


$$
\pi \frac{u}{v_{a}} \sqrt{\frac{M}{F}}
$$

Regarding the rigid body and the forces as constant, and comparing inter se the periods about different screws $a$, on which the body might have been constrained to twist, we see from the result just arrived at that the time for each screw $a$ is proportional to $\frac{u_{\alpha}}{v_{\alpha}}$.

$\S 85$. Property of Harmonic Screws.-As the time of vibration is affected by the position of the screw to which the motion is limited, it becomes of interest to consider how a screw is to be chosen so that the time of vibration shall be a maximum or minimum. With slightly increased generality we may state the problem as follows :-

Given a rigid body, and the forces which act upon it, it is required to select from all the screws of a given screw complex the particular screw or screws on which, if the body be constrained to twist, the time of vibration will be a maximum or minimum, relatively to the time of vibration on the neighbouring screws of the same screw complex.

Take the $n$ principal screws of inertia belonging to the screw complex, as screws of reference, then we have to determine the $n$ co-ordinates of a screw $\boldsymbol{a}$ by the condition that the function $\frac{u_{\alpha}}{v_{\alpha}}$ shall be a maximum or a minimum.

Introducing the value of $u_{\alpha}(\S 67)$, and of $v_{\alpha}(\S 72)$, in terms of the co-ordinates, we have to determine the maximum and minimum of the function-

$$
\frac{A_{11} a_{1}^{2}+\ldots+A_{n n} a_{n}^{2}+2 A_{12} a_{1} a_{2}+2 A_{13} a_{1} \alpha,+\ldots}{u_{1}^{2} a_{1}^{2}+\ldots+u_{n}^{2} a_{n}^{2} .}=x,
$$

Multiplying this equation by the denominator of the 
left-hand side, differentiating with respect to each coordinate successively, and observing thăt the differential co-efficients of $x$ must be zero, we have the $n$ equations :-

$$
\begin{gathered}
\left(A_{11}-u_{1}^{2} x\right) \alpha_{1}+A_{12} \alpha_{2} \ldots,+A_{1 n} \alpha_{n}=0 . \\
\text { \&c., \&c. } \\
A_{n 1} \alpha_{1}+A_{n 2} \alpha_{2} \ldots\left(A_{n n}-u_{n}^{2} x\right) \alpha_{n}=0 .
\end{gathered}
$$

We hence see that there are $n$ screws belonging to each screw complex of the $n^{\text {th }}$ order on which the time of vibration is a maximum or minimum, and by comparison with $\S 74$ we deduce the very interesting result that these $n$ screws are also the harmonic screws.

Taking the screw complex of the sixth order, which of course includes every screw in space, we see that if the body be permitted to twist about one of the six harmonic screws the time of vibration will be a maximum or minimum, as compared with the time of vibration on any adjacent screw.

If the six harmonic screws were taken as the screws of reference, then $u_{a}{ }^{2}$ and $v_{a}{ }^{2}$ would each consist of the sum of six square terms $(\$ 59,72)$. If the co-efficients in these two expressions were proportional, so that $u_{\alpha}^{2}$ only differed from $v_{a}{ }^{2}$ by a numerical factor, we should then find that every screw in space was an harmonic screw, and that the times of vibrations about all these screws were equal. 


\section{CHAPTER X.}

THE DYNAMICS OF A RIGID BODY WHICH HAS FREEDOM OF THE SECOND ORDER.

$\S 86$. The Screw Complex of the Second Order.-When a rigid body is capable of being twisted about two screws $\theta$ and $\phi$, it is capable of being twisted about every screw on the cylindroid $(\theta, \phi)$. If it also appear that the body cannot be twisted about any screw which does not lie on the cylindroid, then the body is said to have freedom of the second order, and the cylindroid is the screw complex of the second order by which the freedom is defined.

Eight numerical data are required for the determination of a cylindroid. We must have four for the specification of the nodal line, two more are required to define the extreme points in which the surface cuts the nodal line, one to assign the direction of one generator, and one to give the pitch of one screw, or the eccentricity of the pitch conic.

Although only eight constants are required to define the cylindroid, yet ten constants must be used in defining two screws $\theta, \phi$, from which the cylindroid could be constructed. The ten constants not only define the cylindroid, but also point out two special screws upon the surface.

$\$ 87$. Applications of Screw Co-ordinates.-We have shown $(\$ 42)$ that if $a, \beta$ be the two screws of a cylindroid, which intersect at right angles, that then the co-ordinates of any screw $\theta$, which makes an angle $l$ with the screw $a$, are :- 
$a_{1} \cos l+\beta_{1} \sin l, \ldots, a_{6} \cos l+\beta_{6} \sin l$,

reference being made as usual to any set of six coreciprocals.

In addition to the examples of the use of these coordinates already given $(\$ 42)$, we may apply them to the determination of that single screw $\theta$ upon the cylindroid $(\alpha, \beta)$, which is reciprocal to a given screw $\eta$.

From the condition of reciprocity we must have:$p_{1} \eta\left(a_{1} \cos l+\beta_{1} \sin l\right)+\ldots+p_{6} \eta_{6}\left(a_{6} \cos l+\beta_{6} \sin l\right)=0$, or,

$$
\varpi_{a \eta} \cos l+\varpi_{\beta \eta} \sin l=0 .
$$

From this $\tan l$ is deduced, and therefore the screw $\theta$ becomes known ( $\$ 28)$.

In general if $2 \sigma_{\eta \theta}$ be the virtual co-efficient between any screw $\eta$ and a screw $\theta$ on the cylindroid, we have :-

$$
\varpi_{\eta \theta}=\varpi_{\alpha \eta} \cos l+\varpi_{\beta \eta} \sin l ;
$$

whence if on each screw $\theta$ a distance be set off from the nodal line equal to half the virtual co-efficient between $\eta$ and $\theta$, the points thus found will lie on a right circular cylinder, of which the equation is; -

$$
x^{2}+y^{2}=\varpi_{\eta^{n}} x+\varpi_{\beta \eta} y .
$$

Thus the screw which has the greatest virtual coefficient with $\eta$ is at right angles to the screw reciprocal to $\eta$, and in general two screws can be found upon the cylindroid which have a given virtual co-efficient with an external screw.

§88. Relation between Two Cylindroids.-We may here notice a curious reciprocal relation between two cylindroids, which is manifested when one condition is satisfied. If a screw can be found on one cylindroid, which 
is reciprocal to a second cylindroid, then conversely a screw can be found on the latter, which is reciprocal to the former. Let the cylindroids be $(\alpha, \beta)$, and $(\lambda \mu)$. If a screw can be found on the former, which is reciprocal to the latter, then we have:-

$p_{1} \lambda_{1}\left(a_{1} \cos l+\beta_{1} \sin l\right)+\ldots+p_{n} \lambda_{n}\left(a_{n} \cos l+\beta_{n} \sin l\right)=0$.

$p_{1} \mu_{1}\left(a_{1} \cos l+\beta_{1} \sin l\right)+\ldots+p_{n} \mu_{n}\left(a_{n} \cos l+\beta_{n} \sin l\right)=0$.

Whence eliminating $l$, we find:-

$$
\varpi_{\alpha \lambda} \varpi_{\beta \mu}-\varpi_{\beta \lambda \varpi_{a \mu}}=0 .
$$

As this relation is symmetrical with regard to the two cylindroids, the theorem has been proved.

$\S 89$. Co-ordinates of Three Screws on a Cylindroid.-The co-ordinates of three screws upon a cylindroid are connected by four independent relations. In fact, two screws define the cylindroid, and the third screw must then satisfy four equations of the form $(\S 22)$. These relations can be expressed most symmetrically in the form of six equations, which also involve three other quantities.

Let $\lambda, \mu, \nu$ be three screws upon a cylindroid, and let $A, B, C$ denote the angles between $\mu, \nu$, between $\nu \lambda$, and between $\lambda \mu$, respectively. If wrenches of intensities $\lambda^{\prime \prime}, \mu^{\prime \prime}, \nu^{\prime \prime}$, on $\lambda, \mu, \nu$, respectively, are in equilibrium, we must have $(\S \mathrm{I} 7)$ :-

$$
\frac{\lambda^{\prime \prime}}{\sin A}=\frac{\mu^{\prime \prime}}{\sin B}=\frac{\nu^{\prime \prime}}{\sin C}
$$

But we have also as a necessary condition that if each wrench be resolved into six component wrenches on six screws of reference, the sum of the intensities of the three components on each screw of reference is zero; whence:- 
$\lambda_{1} \sin A+\mu_{1} \sin B+\nu_{1} \sin C=0$,

$$
\text { \&c., \&c, }
$$

$\lambda_{6} \sin A+\mu_{6} \sin B+v_{6} \sin C=0$.

From these equations we deduce the following corollaries :-

The screw of which the co-ordinates are proportional to $a \lambda_{1}+b \mu_{1}, \ldots, a \lambda_{6}+b \mu_{6}$, lies on the cylindroid $(\lambda, \mu)$, and makes angles with the screws $\lambda, \mu$, of which the sines are inversely proportional to $a$ and $b$.

The two screws, of which the co-ordinates are proportional to $a \lambda_{1} \pm b \mu_{1}, \ldots, a \lambda_{6} \pm b \mu_{6}$, and the two screws $\lambda, \mu$ are respectively parallel to the four rays of a plane harmonic pencil.

$\S 90$. Screw Complex of the Fifth Order and Second Degree.-We have now occasion to make a slight digression from the subject of the present chapter. We have hitherto spoken of the order of a screw complex, and we shall now explain what is to be understood in the use of the word degree. It will be remembered that a screw complex of the $5^{\text {th }}$ order consists of all those screws about which a body having freedom of the $5^{\text {th }}$ order can twist. We may, however, give an analytical definition of such a complex. It appears from $\S 50$ that the six co-ordinates of a screw $\theta$ belonging to a screw complex of the $5^{\text {th }}$ order satisfy that one equation of the first degree which expresses the condition that $\theta$ is reciprocal to the one screw to which the entire complex is reciprocal (\$ 49). Hence we might with perfect generality define a screw complex of the fifth order and first degree to consist of all those screws whose six co-ordinates satisfy one homogeneous equation of the first degree.

The reflective reader may be tempted to inquire into the physical or geometrical meaning of that collec- 
tion of screws whose co-ordinates satisfy one homogeneous equation of the secona degree, and which may be defined to be a screw complex of the fifth order and second degree. We shall develop a few propositions on this subject, which will be useful in what is to follow; but the general discussion of this species of complex, though apparently of great interest, lies beyond the scope of the present volume.

$\S$ 91. Polar Screws.-Let us denote a screw complex of the fifth order and second degree by the equation $U_{\theta}=0$, where $U_{\theta}$ is an homogeneous function of the second degree in the six quantities $\theta_{1}, \ldots, \theta_{6}$.

Let $\eta$ and $\zeta$ derote any two screws. If then (to adopt the fertile principle used by Dr. Salmon*) we substitute in $U=0$ for $\theta_{1}, \theta_{2}, \&$ c., the expressions $l \eta_{1}+m \zeta_{1}$, $l \eta_{2}+m \zeta_{2}$, \&c., we obtain the equation :-

$$
l^{2} U_{\eta}+l m U_{\eta \zeta}+m^{2} U_{\zeta}=0 ;
$$

where $U_{\eta \zeta}$ denotes the expression :-

$$
\zeta_{1} \frac{d U}{d \eta_{1}}+\ldots+\zeta_{6} \frac{d U}{d \eta_{6}} .
$$

Solving the quadratic equation for $l: m$, we obtain two values of this ratio, and hence $(\$ 89)$ we see that two screws belonging to the screw complex $U_{\theta}=0$ can be found on any cylindroid $(\eta, \zeta)$.

If the relation between $\eta$ and $\zeta$ be such, that-

$$
U_{\eta \zeta}=0 \text {, }
$$

the two roots of the equation will be equal in magnitude, and opposite in sign, and hence we deduce the following theorem :-

- Conic Sections, 3rd Edition, p. $\times 34$. 
If the condition $U_{\eta \zeta}=0$ be fulfilled, then the two screws $\eta, \zeta$, and the two screws on the cylindroid $(\eta, \zeta)$, which belong to the complex $U_{\theta}=0$, are parallel to the four rays of an harmonic pencil ( $(89)$.

We can now deduce a result of some importance. If we regard the screw $\eta$ as being given, then the screw $\zeta$ must belong to a screw complex of the fifth order and first degree, which is defined by :-

$$
U_{\eta_{\zeta}}=0 .
$$

This complex may be constructed in the following manner :-Draw any cylindroid through $\eta$, find on this the two screws which belong to $U_{\theta}=0$, then a fourth screw $\zeta$ can be determined by the condition that the set shall be parallel to the rays of an harmonic pencil. The same process repeated for four other cylindroids through $\eta$, will give five screws, by which the screw complex to which $\zeta$ belongs is determined.

It will be observed that in the determination of the screw complex $U_{\eta \zeta}=0$, where $\eta$ is given, no occasion has arisen for making mention of the screws of reference to which the co-ordinates are referred. If, further, it be observed, that all the screws of the complex $U_{\eta \zeta}=0$ are reciprocal to that one screw of which the co-ordinates are proportional to-

$$
\frac{\mathrm{I}}{p_{1}}\left(\frac{d U_{\eta}}{d \eta_{1}}\right), \ldots \frac{\mathrm{I}}{p_{6}} \cdot\left(\frac{d U_{\eta}}{d \eta_{6}}\right),
$$

we have the following theorem :-

If $U_{\theta}=0$ denote a screw complex of the fifth order and second degree, then to every screw $\eta$ corresponds with respect to the screw complex, a polar screw, whose co-ordinates are proportional to-

$$
\frac{\mathrm{I}}{p_{1}}\left(\frac{d U_{\eta_{1}}}{d \eta_{1}}\right) \ldots, \frac{\mathrm{I}}{p_{6}}\left(\frac{d U_{\eta}}{d \eta_{6}},\right)
$$


the relation between $\eta$ and its polar being completely independent of the group of co-reciprocal screws, which have been chosen as the screws of reference.

$\S$ 92. Properties of Screws and their Polars.-We add here a few properties which are, however, not demonstrated, as we shall have no occasion to make use of them. If $\boldsymbol{a}$ and $\beta$ be two screws, and if $\boldsymbol{\eta}$ and $\zeta$ be their polars, with respect to a screw complex of the fifth order and second degree, then, when $a$ is reciprocal to $\zeta$, we shall find that $\beta$ is reciprocal to $\eta$. We may term $a$ and $\beta$ conjugate screws of the complex.

If the discriminant of $U_{\theta}=0$ vanish, there is then a "central" screw of the complex, to which the polars of all other screws are reciprocal.

The equation of the screw complex will reduce to the sum of six square terms when referred to six screws of which each pair are conjugate.

Six screws can be found which coincide with their polars, and these six screws are both conjugate and coreciprocal.

Six screws can be found, every pair of which are conjugate with respect to each of two given screw complexes of the fifth order and second degree.

$\S$ 93. Pitch Complex.-All the screws in space of given pitch $h$ belong to a screw complex of the fifth order and second degree, of which the equation may be written :-

$p_{1} \theta_{1}{ }^{2}+\ldots+p_{6} \theta_{6}{ }^{2}=h\left[\theta_{1}{ }^{2}+\ldots+\theta_{6}{ }^{2}+2 \theta_{1} \theta_{2} \cos \left(\omega_{1} \omega_{2}\right)+\ldots\right]$

where the quantity inside the bracket is really equal to unity, but is introduced for the sake of making the equation homogeneous ( $\S 37$ ). This quantity is denoted by $R$.

This complex is, from the nature of the case, com pletely independent of the screws of reference. The 
polar of a screw $\eta$, with respect to this complex, must be also completely independent of the screws of reference. It is, therefore, obvious that the polar of $\eta$ must be a screw which lies in the same straight line as $\eta$, for symmetry will not permit any reason to be assigned in favour of any other position. The co-ordinates, therefore, of a screw $\zeta$, which lies in the same straight line as $\eta$, but which has a different pitch $p_{\zeta}$, must be equal to :-

$$
A\left(\eta_{1}-\frac{h}{p_{1}} \frac{d R}{d \eta_{1}}\right), \ldots, A\left(\eta_{6}-\frac{h}{p_{6}} \frac{d R}{d \eta_{6}}\right),
$$

where $A$ and $h$ are constants to be determined.

We sacrifice no generality by making the pitch of $\eta$ zero. We shall now write two identical equations. Of these the first expresses that the pitch of $\zeta$ is $p_{\zeta}$, and the second expresses that the virtual co-efficient of $\zeta$ and $\eta$ is $p_{\xi}:-$

$$
\begin{aligned}
& A^{2} p_{1}\left(\eta_{1}-\frac{h}{p_{1}} \cdot \frac{d R}{d \eta_{1}}\right)^{2}+\ldots+A^{2} p_{6}\left(\eta_{6}-\frac{h}{p_{6}} \cdot \frac{d R}{d \eta_{6}}\right)^{2}=p_{5} . \\
& A p_{1} \eta_{1}\left(\eta_{1}-\frac{h}{p_{1}} \frac{d R}{d \eta_{1}}\right)+\ldots+A p_{6} \eta_{6}\left(\eta_{6}-\frac{h}{p_{6}} \cdot \frac{d R}{d_{6} \eta}\right)=\frac{1}{2} p_{5}
\end{aligned}
$$

Remembering that $p_{1} \eta_{1}{ }^{2}+\ldots+p_{6} \eta_{6}{ }^{2}=0$, and also that $R$ is an homogeneous function of the second order, and that, therefore, by Euler's theorem* :-

$$
\eta_{1} \frac{d R}{d \eta_{1}}+\ldots+\eta_{6} \frac{d R}{d \eta_{6}}=2 R=2,
$$

we have :-

$$
\begin{aligned}
-4 A^{2} h+A^{2} h^{2}\left(\frac{\mathrm{I}}{p_{1}}\left(\frac{d R}{d \eta_{1}}\right)^{2}+\ldots+\frac{\mathrm{I}}{p_{6}}\left(\frac{d R}{d \eta_{6}}\right)^{2}\right) & =p_{\zeta} . \\
-4 A h & =p_{\zeta} .
\end{aligned}
$$

* Williamson's Differential Calculus, 2nd Ed., p. II3. 
Whence we deduce $A h=-\frac{p_{\zeta}}{4}$, and since when $p_{\zeta}=0$ the screw must reduce to $\eta$, we find $A=\mathrm{I}$.

We, therefore, deduce the following theorem:-If $\eta_{1}, \ldots . \eta_{6}$, be the co-ordinates of a screw of zero pitch, then the co-ordinates of a screw $\zeta$, of pitch $p_{\zeta}$, upon the same straight line as the screw $\eta$ are equal to the six quantities :-

$$
\eta_{1}+\frac{p_{\zeta}}{4 p_{1}} \frac{d R}{d \eta_{1}}, \ldots, \eta_{6}+\frac{p_{\zeta}}{4 p_{6}} \frac{d R}{d \eta_{6}},
$$

in which

$$
R=\eta_{1}{ }^{2}+\ldots .+\eta_{6}{ }^{2}+2 \eta_{1} \eta_{2} \cos \left(\omega_{1} \omega_{2}\right)+2 \eta_{1} \eta_{3} \cos \left(\omega_{1} \dot{\omega}_{3}\right)+\ldots=\mathrm{I} \text {. }
$$

We may remark that the co-ordinates of a screw of infinite pitch, parallel to $\zeta$, are proportional to :-

$$
\frac{\mathrm{I}}{p_{1}} \frac{d R}{d \eta_{1}}, \ldots \frac{\mathrm{I}}{p_{6}} \frac{d R}{d \eta_{6}} \text {. }
$$

We can also prove that $\frac{1}{2} \frac{d R}{d \eta_{1}}$ is the cosine of the angle between the screw $\eta$, and the screw of reference $\omega_{1}$. Let $O$ be this angle, and let $d$ be the shortest distance between $\eta$ and $\dot{\omega}_{1}$. Then we have $(\S 35)$ :-

$$
2 p_{1}\left(\eta_{1}+\frac{p_{\zeta}}{4 p_{1}} \frac{d R}{d \eta_{1}}\right)=\left(p_{1}+p_{\xi}\right) \cos O-d \sin O,
$$

and as this must be true whatever may be the value of $p_{\zeta}$, it follows that :-

$$
\frac{\mathrm{I}}{2} \frac{d R}{d \eta_{1}}=\cos O
$$

We also have the identity :-

$$
\frac{1}{p_{1}}\left(\frac{d R}{d \eta_{1}}\right)^{2}+\ldots+\frac{1}{p_{6}}\left(\frac{d R}{d \eta_{6}}\right)^{2}=0 .
$$


From this we see that three of the pitches of a set of six co-reciprocals must be + , and three must be -.* For, suppose that the pitches of four of the co-reciprocals had the same sign, and let $\eta$ be a screw perpendicular to the two remaining co-reciprocals, then the identity just written would reduce to the sum of four positive terms equal zero, which is absurd.

$\S 94$. Screws on One Line.-There is one case in which a body has freedom of the second order that demands special attention. Suppose the two given screws $\theta, \phi$, about which the body can be twisted, happen to lie on the same straight line, then the cylindroid becomes illusory. If the amplitudes of the two twists be $\theta, \phi^{\prime}$, then the body will have received a rotation $\theta^{\prime}+\phi^{\prime}$, accompanied by a translation $\theta^{\prime} p_{\theta}+\phi^{\prime} p_{\phi}$. This movement is really identical with a twist on a screw of which the pitch is :-

$$
\frac{\theta^{\prime} p_{\theta}+\phi^{\prime} p_{\phi}}{\theta^{\prime}+\phi^{\prime}}
$$

Since $\theta^{\prime}, \phi^{\prime}$ may have any ratio, we see that, under these circumstances, the screw complex which defines the freedom consists of all the screws with pitches ranging from $-\infty$ to $+\infty$, which lie along the given line. It follows ( $(93)$, that the co-ordinates of all the screws about which the body can be twisted are to be found by giving $p_{\zeta}$ all the values from $-\infty$ to $+\infty$ in the expressions :-

$$
\eta_{1}+\frac{p_{5}}{4 p_{1}} \cdot \frac{d R}{d \eta_{1}}, \ldots, \eta_{6}+\frac{p_{5}}{4 p_{6}} \frac{d R}{d \eta_{6}}
$$

* This interesting theorem was communicated to me by Dr. Klein, who had proved it as a property of the parameters of "six fundamental complexes in involution" (Math. Ann. Band. ii., p. 204). 
$\S 95$. Displacement of a Point. - Let $P$ be a point, and let $\alpha, \beta$ be any two screws upon a cylindroid. If a body to which $P$ is attached receive a small twist about $\alpha$, the point $P$ will be moved to $P^{\prime}$. If the body receive a small twist about $\beta$, the point $P$ would be moved to $P^{\prime \prime}$. Then whatever be the screw $\gamma$ on the cylindroid about which the body be twisted, the point $P$ will still be displaced in the plane $P P^{\prime} P^{\prime \prime}$.

For the twist about $\gamma$ can be resolved into two twists about $\alpha$ and $\beta$, and therefore every displacement of $P$ must be capable of being resolved along $P P^{\prime}$ and $P P^{\prime \prime}$.

Thus through every point $P$ in space a plane can be drawn to which the small movements of $P$, arising from twists about the screws on a given cylindroid are confined. The simplest construction for this plane is as follows :-Draw through the point $P$ two planes, each containing one of the screws of zero pitch; the intersection of these planes is normal to the required plane through $P$.

The construction just given would fail if $P$ lay upon one of the screws of zero pitch. The movements of $P$ must then be limited, not to a plane, but to a line. The line is found by drawing a normal to the plane passing. through $P$, and through the other screw of zero pitch.

We thus have the following curious property of the lines of zero pitch, viz., that a point in the rigid body on the line of zero pitch will commence to move in the same direction whatever be the screw on the cylindroid about which the twist is imparted.

This easily appears otherwise. Appropriate twists about any two screws, $\alpha$ and $\beta$, can compound into a twist about the screw of zero pitch $\lambda$, but the twist about $\lambda$ cannot disturb a point on $\lambda$. Therefore a twist about $\beta$ must be capable of moving a point originally on $\lambda$ back to its position before it was disturbed by $a$. Therefore the 
twists about $\beta$ and $a$ must move the point in the same direction.

$\S$ 96. Properties of the Pitch Conic.-Since the pitch of a screw on a cylindroid is proportional to the inverse square of the parallel diameter of the pitch conic ( $\$ 20)$, the asymptotes must be parallel to the screws of zero pitch ; also since a pair of reciprocal screws are parallel to a pair of conjugate diameters ( $(42)$, it follows that the two screws of zero pitch, and any pair of reciprocal screws, are parallel to the rays of an harmonic pencil.* If the pitch conic be an ellipse, there are no real screws of zero pitch. If the pitch conic be a parabola, there is but one screw of zero pitch, and this must be one of the two screws which intersect at right angles. Since this screw is reciprocal to itself, as well as to the screw it intersects, it must be reciprocal to every screw on the cylindroid ( $(24)$. This is the only case in which a screw on the cylindroid is reciprocal to the cylindroid.

$\$ 97$. Equilibrium of a Body with Freedom of the Second Order.-We shall now consider more fully the conditions under which a body which has freedom of the second order is in equilibrium. The necessary and sufficient condition is, that the forces which act upon the body shall constitute a wrench upon a screw which is reciprocal to the cylindroid which defines the freedom of the body.

It has been shown ( $\S 25)$, that the screws which are reciprocal to a cylindroid exist in such profusion, that through every point in space a cone of the second order can be drawn, of which the entire superficies is made up of such screws. We shall now examine the distribution of pitch upon such a cone.

* Salmon's Conic Sections, 3rd Edition, p. 273. 
The pitch of each reciprocal screw is equal in magnitude, and opposite in sign, to the pitches of the two screws of equal pitch, in which it intersects the cylindroid ( $\$ 24)$. Now, the greatest and least pitches of the screws on the cylindroid are $p$ and $p_{\beta}(\S 20)$. For the quantity $p_{a} \cos ^{2} l+p_{\beta} \sin ^{2} l$ is always intermediate between $p_{a} \cos ^{2} l+p_{a} \sin ^{2} l$ and $p_{\beta} \cos ^{2} l+p \sin { }^{2} l$. Hence it follows that the generators of the cone which meet the cylindroid in three real points must have pitches intermediate between $p_{a}$ and $p_{\beta}$. It is also to be observed that, as only one line can be drawn through the vertex of the cone to intersect any two given screws on the cylindroid, so only one screw of any given pitch can be found on the reciprocal cone.

One screw can be found upon the reciprocal cone of every pitch from $-\infty$ to $+\infty$. The line drawn through the vertex parallel to the nodal line is a generator of the cone to which infinite pitch must be assigned. Setting out from.this line around the cone the pitch gradually decreases to zero, then becomes negative, and increases to negative infinity, when we reach the line from which we started. We may here notice that when a screw has infinite pitch, we may regard the infinity as either + or - indifferently. If we conceive distances marked upon each generator of the cone from the vertex, equal to the pitch of that generator, then the parallel to the nodal line drawn from the vertex forms an asymptote to the curve so traced upon the cone. It is manifest that we must admit the cylindroid to possess imaginary screws, whose pitch is nevertheless real.

The reciprocal cone drawn from a point to a cylindroid, is decomposed into two planes, when the point lies upon the cylindroid. The first plane is normal to the generator passing through the point. Every line in this plane must, when it receives the proper pitch, be 
a reciprocal screw. The second plane is that drawn through the point, and through the other screw of equal pitch on the cylindroid, to that which passes through the point.

We have, therefore, solved in the most general manner the problem of the equilibrium of a rigid body with two degrees of freedom. We have shown that the necessary and sufficient condition is, that the resultant wrench be about a screw reciprocal to the cylindroid expressing the freedom, and we have seen the manner in which the reciprocal screws are distributed through space. We now add a few particular cases.

$\S 98$. Particular Cases. - A body which has two degrees of freedom is in equilibrium under the action of a force, whenever the line of action of the force intersects both the screws of zero pitch upon the cylindroid.

If a body acted upon by gravity have freedom of the second order, the necessary and sufficient condition of equilibrium is, that the vertical through the centre of inertia shall intersect both of the screws of zero pitch.

A body which has freedom of the second order will be in equilibrium, notwithstanding the action of a couple, provided the axis of the couple be parallel to the nodal line of the cylindroid.

A body which has freedom of the second order will remain in equilibrium, notwithstanding the action of a wrench about a screw of any pitch on the nodal line of the cylindroid.

$\S 99$. The Impulsive Cylindroid and the Instantaneous Cylindroid.-A rigid body $M$ is at rest in a position $P$, from which it is either partially or entirely free to move. If $M$ receive an impulsive wrench about a screw $X_{1}$, it will commence to twist about an instantaneous screw $A_{1}$, if, however, the impulsive wrench had been about $X_{2}$ or $X_{3}$ ( $M$ being in either case at rest in the position $P$ ) 
the instantaneous screw would have been $A_{2}$, or $A_{3}$. Then we have the following theorem :-

If $X_{1}, X_{2}, X_{3}$ lie upon a cylindroid $S$ (which we may call the impulsive cylindroid), then $A_{1}, A_{2}, A_{3}$ lie on a cylindroid $S^{\prime}$ (which we may call the instantaneous cylindroid).

For if the three wrenches have suitable intensities they may equilibrate, since they are cocylindroidal: when this is the case the three instantaneous twist velocities must, of course, neutralize; but this is only possible if the instantaneous screws be cocylindroidal ( $\$ 63$ ).

If we draw a pencil of four lines through a point parallel to four generators of a cylindroid, the lines forming the pencil will lie in a plane. We may define the anharmonic ratio of four generators on a cylindroid to be the anharmonic ratio of the parallel pencil. We shall now prove the following theorem*:-

The anharmonic ratio of four screws on the impulsive cylindroid is equal to the anharmonic ratio of the four corresponding screws on the instantaneous cylindroid.

Before commencing the proof we remark that,

If an impulsive wrench of intensity $F$ acting on the screw $X$ be capable of producing the unit of twist velocity about $A$, then a wrench of intensity $F_{\omega}$ on $X$ will produce a twist velocity $\omega$ about $A$.

Let $X_{1}, X_{2}, X_{3}, X_{4}$ be four screws on the impulsive cylindroid, the intensities of the wrenches appropriate to which are $F_{1} \omega_{1}, F_{2} \omega_{2}, F_{3} \omega_{3}, F_{4} \omega_{4}$. Let the four corresponding instantaneous screws be $A_{1}, A_{2}, A_{3}, A_{4}$, and the twist velocities be $\omega_{1}, \omega_{2}, \omega_{3}, \omega_{\downarrow}$. Let $\phi_{m}$ be the angle

* This theorem is an illustration of the important bearings of the Theory of Correspondenee on the Theory of Screws. 
on the impulsive cylindroid defining $X_{m}$, and let $\theta_{m}$ be the angle on the instantaneous cylindroid defining $A_{m}$.

If three impulsive wrenches equilibrate, the corresponding twist velocities neutralise : hence ( $\S$ I 7 ) it must be possible for certain values of $\omega_{1}, \omega_{2}, \omega_{3}, \omega_{4}$ to satisfy the following equations :-

$$
\begin{aligned}
& \frac{\omega_{1}}{\sin \left(\theta_{2}-\theta_{3}\right)}=\frac{\omega_{2}}{\sin \left(\theta_{3}-\theta_{1}\right)}=\frac{\omega_{3}}{\sin \left(\theta_{1}-\theta_{2}\right)}, \\
& \frac{F_{1} \omega_{1}}{\sin \left(\phi_{2}-\phi_{3}\right)}=\frac{F_{2} \omega_{2}}{\sin \left(\phi_{3}-\phi_{1}\right)}=\frac{F_{3} \omega_{3}}{\sin \left(\phi_{1}-\phi_{2}\right)}, \\
& \frac{\omega_{2}}{\sin \left(\theta_{3}-\theta_{4}\right)}=\frac{\omega_{3}}{\sin \left(\theta_{4}-\theta_{2}\right)}=\frac{\omega_{4}}{\sin \left(\theta_{2}-\theta_{3}\right)}, \\
& \frac{F_{2} \omega_{2}}{\sin \left(\phi_{3}-\phi_{4}\right)}=\frac{F_{3} \omega_{3}}{\sin \left(\phi_{4}-\phi_{2}\right)}=\frac{F_{4} \omega_{4}}{\sin \left(\phi_{2}-\phi_{3}\right)},
\end{aligned}
$$

whence

$$
\frac{\sin \left(\theta_{1}-\theta_{2}\right) \sin \left(\theta_{3}-\theta_{4}\right)}{\sin \left(\theta_{3}-\theta_{1}\right) \sin \left(\theta_{4}-\theta_{2}\right)}=\frac{\sin \left(\phi_{1}-\phi_{2}\right) \sin \left(\phi_{3}-\phi_{4}\right)}{\sin \left(\phi_{3}-\phi_{1}\right) \sin \left(\phi_{4}-\phi_{2}\right)},
$$

which proves the theorem.

If, therefore, we are given three screws on the impulsive cylindroid, and the corresponding three screws on the instantaneous cylindroid, the connexion between every other corresponding pair is geometrically, determined.

$\S$ 100. Reaction of Constraints. - Whatever the constraints may be, their reaction produces an impulsive wrench $R_{1}$ upon the body at the moment when the impulsive wrench $X_{1}$ acts. The two wrenches $X_{1}$ and $R_{1}$ compound into a third wrench $Y_{1}$. If the body were free, $Y_{1}$ is the impulsive wrench to which the instantaneous screw $A_{1}$ would correspond. Since $X_{1}, X_{2}, X_{3}$ are cocylindroidal, $A_{1}, A_{2}, A_{3}$ must be cocylindroidal, 
and therefore also must be $Y_{1}, Y_{2}, Y_{3}$. The nine wrenches $X_{1}, X_{2}, X_{3}, R_{1}, R_{2}, R_{3},-Y_{1},-Y_{2},-Y_{3}$ must equilibrate; but if $X_{1}, X_{2}, X_{3}$ equilibrate, then the twist velocities about $A_{1}, A_{2}, A_{3}$ must neutralize, and therefore the wrenches about $Y_{1}, Y_{2}, Y_{3}$ must equilibrate. Hence $R_{1}, R_{2}, R_{3}$ equilibrate, and are therefore cocylindroidal.

Following the same line of proof used in the last section, we can show that

If impulsive wrenches on any four cocylindroidal screws act upon a partially free rigid body, the four corresponding initial reactions of the constraints also constitute wrenches about four cocylindroidal screws; and, further, the anharmonic ratios of the two groups of four screws are equal.

$\S$ IOI. Principal Screws of Inertia.-If a quiescent body with freedom of the second order receive impulsive wrenches on three screws $X_{1}, X_{2}, X_{3}$ on the cylindroid which expresses the freedom, and if the corresponding instantaneous screws on the same cylindroid be $A_{1}, A_{2}, A_{3}$, then the relation between any other impulsive screw $X$ on the cylindroid and the corresponding instantaneous screw $A$ is completely defined by the condition that the anharmonic ratio of $X, X_{1}, X_{2}, X_{3}$ is equal to the anharmonic ratio of $A, A_{1}, A_{2} A_{3}$.

Now, if three rays parallel to $X_{1}, X_{2}, X_{3}$ be drawn from a point, and also three rays parallel to $A_{1}, A_{2}, A_{3}$, then it is well known* that the problem to determine a ray $Z$ such that the anharmonic ratio of $Z, A_{1}, A_{2}, A_{3}$ is equal to that of $Z, X_{1}, X_{2}, X_{3}$, admits of two solutions. There are, therefore, two screws on a cylindroid which possess the property that an impulsive wrench on one of these screws will cause the body to commence to twist about the same screw.

* Chasles, passim. See also Townsend's Modern Geometry, vol. ii., p. 246. 
We have thus arrived by a special process at the two principal screws of inertia posssesed by a body which has freedom of the second order. This is, of course, a particular case of the general theorem of $\S 5 \mathrm{I}$. We shall show in the next section how these screws can be determined in another manner.

$\S$ 102. The Ellipse of Inertia.-We have seen ( $\$ 59)$ that a linear parameter $u_{\alpha}$ may be conceived appropriate to each screw $a$ of a comiplex, so that when the body is twisting about the screw $a$ with the unit of twist velocity, the kinetic energy is found by multiplying the mass of the body into the square of the line $u_{a}$.

We are now going to consider the distribution of this magnitude on $u_{\alpha}$ the screws of a cylindroid. If we denote by $u_{1}, u_{2}$ the values of $u_{\alpha}$ for any pair of conjugate screws of inertia on the cylindroid ( $\$ 54)$, and if by $\boldsymbol{a}_{1}, \boldsymbol{a}_{2}$ we denote the intensities of the components on the two conjugate screws of a wrench of unit intensity on $a$, we have $(\S 67)-$

$$
u_{\alpha}^{2}=u_{1}^{2} \alpha_{1}^{2}+u_{2}^{2} a_{2}^{2} .
$$

From the centre of the cylindroid draw two lines parallel to the pair of conjugate screws of inertia, and with these lines as axes of $x$ and $y$ construct the ellipse of which the equation is

$$
u_{1}^{2} x^{2}+u_{2}^{2} y^{2}=H,
$$

where $H$ is any constant. If $r$ be the radius vector in this ellipse, we have

$$
\frac{x}{r}=a_{1} \text { and } \frac{y}{r}=a_{2}
$$

whence by substitution we deduce

$$
u_{\alpha}^{2}=\frac{H}{r^{2}},
$$

which proves the following theorem:- 
The linear parameter $u_{\alpha}$ on any screw of the cylindroid is inversely proportional to the parallel diameter of a certain ellipse, and a pair of conjugate screws of inertia on the cylindroid are parallel to a pair of conjugate diameters of the same ellipse. This ellipse may be called the ellipse of inertia.

The major and minor axes of the ellipse of inertia are parallel to screws upon the cylindroid, which for a given twist velocity correspond to a maximum and minimum kinetic energy.

An impulsive wrench on a screw $\boldsymbol{\eta}$ acts upon a quiescent rigid body which has freedom of the second order. It is required to determine the screw $\theta$ on the cylindroid expressing the freedom about which the body will commence to twist.

The ellipse of inertia enables us to solve this problem with great facility. Determine that one screw $\phi$ on the cylindroid which is reciprocal to $\boldsymbol{\eta}(\S 28)$. Draw a diameter $D$ of the ellipse of inertia parallel to $\phi$. Then the required screw $\theta$ is simply that screw on the cylindroid which is parallel to the diameter conjugate to $D$ in the ellipse of inertia.

The converse problem, viz., to determine the screw $\eta$, an impulsive wrench on which would make the body commence to twist about $\theta$ is indeterminate. Any screw in space which is reciprocal to $\phi$ would fulfil the required condition (§54).

We have seen in $\S 66$ that an impulsive wrench on any screw in space may always be replaced by a precisely equivalent wrench upon the cylindroid which expresses the freedom. We are now going to determine the screw $\eta$, on the cylindroid of freedom, an impulsive wrench on which would make the body twist about a given screw $\theta$ on the same cylindroid. This can be easily determined with the help of the pitch conic; for we have seen $(\$ 42)$ that a pair of reciprocal screws on 
the cylindroid of freedom are parallel to a pair of conjugate diameters of the pitch conic. The construction is therefore, as follows:-Find the diameter $A$ which is conjugate, with respect to the ellipse of inertia to the diameter parallel to the given screw $\theta$. Next find the diameter $B$ which is conjugate to the diameter $A$ with respect to the pitch conic. The screw on the cylindroid parallel to the line $B$ thus determined is the required screw $\eta$.

Two concentric ellipses have one pair of common conjugate diameters. In fact, the four points of intersection form a parallelogram, to the sides of which the pair of common conjugate diameters are parallel. We can now interpret physically the common conjugate diameters of the pitch conic, and the ellipse of inertia. The two screws on the cylindroid parallel to these diameters are conjugate screws of inertia, and they are also reciprocal ; they are, therefore, the principal screws of inertia, to which we have been already conducted ( $\$$ Ior).

If the distribution of the material of the body bear certain relations to the arrangement of the constraints, we can easily conceive that the pitch conic and the ellipse of inertia might be both similar and similarly situated. Under these exceptional circumstances it appears that every screw of the cylindroid would possess the property of a principal screw of inertia.

$\S$ 103. The Ellipse of the Potential.-We are now to consider another ellipse, which, though possessing many useful mathematical analogies to the ellipse of inertia, is yet widely different from a physical point of view. We have introduced $(\S 72)$ the conception of the linear magnitude $v_{a}$, the square of which is proportional to the work done in effecting a twist of given amplitude about a screw $\boldsymbol{a}$ from a position of stable equilibrium under the influence of a system of forces. We now propose 
to consider the distribution of the parameter $v_{\alpha}$ upon the screws of a cylindroid. It appears from $(\S 72)$ that if $v_{1}, v_{2}$ denote the values of the quantity $v_{\alpha}$ for each of two conjugate screws of the potential, and if $\boldsymbol{a}_{1}, \boldsymbol{a}_{2}$ denote the intensities of the components on the two conjugate screws of a wrench of unit intensity on a screw $a$, which lies upon the cylindroid, that then-

$$
v_{\alpha}^{2}=v_{1}^{2} a_{1}^{2}+v_{2}^{2} a_{2}^{2} \text {. }
$$

From the centre of the cylindroid draw two straight lines parallel to the pair of conjugate screws of the potential, and with these lines as axes of $x$ and $y$ construct the ellipse, of which the equation is-

$$
v_{1}^{2} x^{2}+v_{2}^{2} y^{2}=H
$$

where $H$ is any constant. If $r$ be the radius vector in this ellipse, we have-

$$
\frac{x}{r}=a_{1} \text { and } \frac{y}{r}=a_{2} ;
$$

whence by substitution we deduce-

$$
v_{a}^{2}=\frac{H}{r^{2}}
$$

which proves the following theorem :-

The linear parameter $v_{a}$ on any screw of the cylindroid is inversely proportional to the parallel diameter of a certain ellipse, and a pair of conjugate screws of the potential are parallel to a pair of conjugate diameters of the same ellipse.

This ellipse may be called the ellipse of the potential.

The major and minor axes of the ellipse of the potential are parallel to screws upon the cylindroid, which, for a twist of given amplitude, correspond to a maximum and minimum potential energy.

When the body is slightly displaced from its position of equilibrium by the action of a wrench of given small intensity on a given screw $\eta$, the twist which the body executes in assuming its new position is per- 
formed about a screw $\theta$, which can very simply be constructed by the ellipse of the potential. Determine the screw $\phi$ (on the cylindroid of freedom) which is reciprocal to $\eta(\S 28)$, then $\phi$, and the required screw $\theta$, are parallel to a pair of conjugate diameters of the ellipse of the potential.

The common conjugate diameters of the pitch conic, and the ellipse of the potential, are parallel to the two screws on the cylindroid, which we have designated the principal screws of the potential ( $\$ 7 \mathrm{I})$.

When a body is displaced from its position of equilibrium by a small wrench upon a principal screw of the potential, then the body moves to the new position which is required in its altered circumstances by a small twist about the same screw.

$\S$ 104. Harmonic Screws.-The common conjugate diameters of the ellipse of inertia, and the ellipse of the potential, are parallel to the two harmonic screws on the cylindroid ( $\$ 74)$. This is evident, because the pair of screws thus determined are conjugate screws both of inertia and of the potential.

If the body be displaced by a twist about one of the harmonic screws, and be then abandoned to the influence of the forces, the body will continue for ever to perform twist oscillations about that screw.

If the ellipse of inertia, and the ellipse of the potential, be similar, and similarly situated, then every screw on the cylindroid of freedom will be an harmonic screw.

\$ 105. Exceptional Case.-We have now to consider the modifications which the results we have arrived at undergo when the cylindroid becomes illusory in the case considered ( $\$ 94)$.

Suppose that $\xi$ and $\zeta$ were a pair of conjugate screws of inertia on the straight line about which the body was free to rotate and slide independently. Then taking 
the six absolute principal screws of inertia* as screws of reference, we must have ( $(66)$ -

$$
\Sigma p_{1}^{2}\left(\eta_{1}+\frac{p_{\xi}}{4 p_{1}} \cdot \frac{d R}{d \eta_{1}}\right)\left(\eta_{1}+\frac{p_{\zeta}}{4 p_{1}} \cdot \frac{d R}{d \eta_{1}}\right)=0
$$

where $\eta$ denotes the screw of zero pitch on the same straight line.

Expanding this equation, and reducing, we find-

$$
u^{2}+\frac{\mathrm{I}}{4}\left(p_{\xi}+p_{\zeta}\right)\left(\Sigma p_{1} \eta_{1} \frac{d R}{d \eta_{1}}\right)+\frac{\mathrm{I}}{\mathrm{I} 6} p_{\xi} p_{\zeta}\left(\Sigma\left(\frac{d R}{d \eta_{1}}\right)^{2}\right)=0 .
$$

This result can be much simplified. By comparing $\S 37$ and $\S 52$, it appears that-

$$
R=\left(\eta_{1}+\eta_{2}\right)^{2}+\left(\eta_{3}+\eta_{4}\right)^{2}+\left(\eta_{5}+\eta_{6}\right)^{2} .
$$

and therefore-

$$
\Sigma p_{1} \eta_{1} \frac{d R}{d \eta_{1}}=2 \Sigma p_{1} \eta_{1}^{2}=2 p_{\eta}=0
$$

Hence we can prove that the product of the pitches of two conjugate screws of inertia is constant, and is equal to minus the square of the radius of gyration about the common axis of the screws.

$\S$ 106. Reaction of Constraints.-We shall now consider the following problem :-A body which is free to twist about all the screws of a cylindroid $C$ receives an impulsive wrench on a certain screw $\eta$. It is required to find the screw $\lambda$, a wrench on which constitutes the impulsive reaction of the constraints. Let $C^{\prime}$ represent the cylindroid which, if the body were perfectly free, would form the locus of those screws, impul-

* We shall often find it convenient to designate the six principal screws of inertia of a free rigid body $(\$ 52)$ by the phrase abolute principal screzes of inertia. 
sive wrenches on which correspond to all the screws of $C$ as instantaneous screws. Since a wrench on $\eta$, and one on $\lambda$, make the body twist about some screw on $C$, it follows that the cylindroid $(\eta, \lambda)$ must have a screw $\rho$ in common with $C^{\prime}$. The wrench on $\lambda$ might be resolved into two. one on $\eta$, and the other on $\rho$, and the latter might be again resolved into two wrenches on any two screws of $C^{\prime}$. It therefore follows that $\lambda$ must belong to the screw complex of the third order, which may be defined by $\eta$, and by any two screws from $C^{\prime}$. Take any three screws reciprocal to this complex, and any two screws on $C$. We have then five screws to which $\lambda$ is reciprocal, and it is therefore geometrically determined (§ 28).

When $\lambda$ is found, the cylindroid $(\eta \lambda)$ can be drawn, and thus $\rho$ is determined. The position of $\rho$ on $C^{\prime}$ will point out the screw on $C$, about which the body will commence to twist, while the position of $\rho$ on $(\eta, \lambda)$, and the known intensity of the wrench on $\eta$, will determine the intensity of the wrench on $\lambda$.

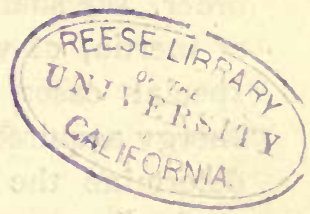




\section{CHAPTER XI.}

THE DYNAMICS OF A RIGID BODY, WHICH HAS FREEDOM OF THE THIRD ORDER.

§ 107. Introduction.-The dynamics of a rigid body which has freedom of the third order, possesses a special claim to attention, for, included as a particular case, we have the celebrated problem of the rotation of a rigid body about a fixed point. In the theory of screws the screw complex of the third order is characterised by the feature that the reciprocal screw complex is also of the third order, and this is a fertile source of interesting theorems.

We shall first study the screw complex of the third order, and its reciprocal. We shall then show how the instantaneous screw, corresponding to a given impulsive screw, can be determined for a rigid body whose movements are prescribed by any screw complex of the third order. We shall also point out the three principal screws of inertia, of which the three principal axes are only special cases, and we shall determine the kinetic energy acquired by a given impulse. Finally, we shall determine the three harmonic screws, and we shall apply these principles to the discussion of the small oscillations of a rigid body about a fixed point under the influence of gravity.

A screw complex of the first order consists of course of one screw. A screw complex of the second order consists of all the screws on a certain ruled surface (the cylindroid). Ascending one step higher, we find that in a screw complex of the third order the screws are so 
numerous that a finite number (three) can be drawn through every point in space. In the screw complex of the fourth order a cone of screws can be drawn through every point, while to a screw complex of the fifth order belongs a screw of suitable pitch on every straight line in space.

$\S$ 108. Screw Complex of the Third Order.-We shall now consider the collocation of the screws in space which constitute a screw complex of the third order. A free rigid body can receive six independent displacements. Its position is, therefore, to be specified by six co-ordinates. If, however, the body be so constrained that its six co-ordinates must always satisfy three equations of condition, there are then only three really independent co-ordinates, and any position possible for a body so circumstanced may be attained by twists about three fixed screws, provided that twists about these screws are permitted by the constraints.

Let $A$ be an initial position of a rigid body $M$. Let $M$ be moved from $A$ to a closely adjacent position, and let $x$ be the screw by twisting about which this movement has been effected; similarly let $y$ and $z$ be the two screws, twists about which would have brought the body from $A$ to two other adjacent positions. We thus have three screws $x, y, z$, which completely specify the circumstances of the body so far as its capacity for movement is considered.

Since $M$ can be twisted about each and all of $x, y, z$, it must be capable of twisting about a doubly infinite number of other screws. For suppose that by twists of amplitude $x^{\prime}, y^{\prime}, z^{\prime}$, the final position $V$ is attained. This position could have been reached by twisting about $v$, so as to come from $A$ to $V$ by a single twist. As the ratios of $x^{\prime}$ to $y^{\prime}$, and $z^{\prime}$, are arbitrary, 
and as a change in either of these ratios changes $v$, the number of $v$ screws is doubly infinite.

All the screws of which $v$ is a type form what we call a screw complex of the third order. We shall often denote this screw complex by the symbol $S$.

$\S$ 109. The Reciprocal Screw Complex.-A wrench which acts on a screw $\eta$ will not be able to disturb the equilibrium of $M$, provided $\eta$ be reciprocal to $x, y, z$. If, therefore, $\eta$ be reciprocal to three screws of the complex $S$, it will be reciprocal to every screw of $S$. Since $\eta$ has thus only three conditions to satisfy in order that it may be reciprocal to $S$, and since five quantities determine a screw, it follows that $\eta$ may be anyone of a doubly infinite number of screws which we may term the reciprocal screw complex $S^{\prime}$. Remembering the property of reciprocal screws ( $\$ 22)$ we have the following theorem ( $\$ 47)$.

A body only free to twist about all the screws of $S$ cannot be disturbed by a wrench on any screw of $S^{\prime \prime}$; and, conversely, a body only free to twist about the screws of $S^{\prime}$ cannot be disturbed by a wrench on any screw of $S$.

The reaction of the constraints by which the freedom is prescribed constitutes a wrench on a screw of $S^{\prime \prime}$.

$\S$ I IO. Distribution of the Screws. - To present a clear picture of all the movements which the body is competent to execute, it will be necessary to examine the mutual connexion of the doubly infinite number of screws which form the screw complex. It will be most convenient in the first place to classify the screws in the complex according to their pitches; the first theorem to be proved is that all the screwes of given pitch $+\mathrm{k}$ lie upon a hyperboloid of which they form one system of generators, while the other system of generators with the pitch $-k$ belong to the recifrocal screw complex $\mathrm{S}^{\prime}$. 
This is proved as follows:-Draw three screws $p, q, r$, of pitch $+k$ belonging to $S$. Draw three screws $l, m, n$, each of which intersects the three screws $p, q, r$, and attribute to each of $l, m, n$, a pitch $-k$. Since two intersecting screws of equal and opposite pitches are reciprocal, it follows that $p, q, r$, must all be reciprocal to $l, m, n$. Hence, since the former belong to $S$, the latter must belong to $S^{\prime}$. Every other screw of pitch $+k$ intersecting $l, m, n$, must be reciprocal to $S^{\prime}$, and must therefore belong to $S$.

But the locus of a straight line which intersects three given straight lines is an hyperboloid of one sheet, ${ }^{*}$ and hence the required theorem has been proved.

§III. The Pitch Quadric.-There is one member of this family of hyperboloids which is of exceptional in terest. We allude to that which is the locus of the screwes' of zero pitch belonging to the screw complex. As the quadric under consideration possesses a very important property ( $($ I I 2 ) besides that of being the locus of the screws of zero pitch, it is desirable to denote it by the special phrase pitch quadric.

We shall now determine the equation of the pitch quadric. Let one of the principal axes of the pitch quadric be denoted by $x$, this will intersect the surface in two points through each of which a pair of generators can be drawn. One generator of each pair will belong to $S$, and the other to $S^{\prime}$. Each pair of generators will be parallelt to the asymptotes of the section of the pitch quadric made by the plane containing the remaining principal axes $y$ and $z$. Let $\mu, \nu$ be the two generators

* Salmon's Analytic Geometry of Three Dimensions, p. 77.

+ Salmon, loc. cit., p. 72. 
belonging to $S$, then lines bisecting internally and externally the angle between two lines in the plane of $y$ and $z$, parallel to $\mu, \nu$ will be two of the principal axes of the pitch quadric. Draw the cylindroid $(\mu \nu)$ Now the two screws of zero pitch on the cylindroid are equidistant from the centre of the cylindroid, and the two rectangular screws of the cylindroid bisect internally and externally the angle between the lines parallel to the screws of zero pitch. Hence it follows that the two rectangular screws of the cylindroid $(\mu \nu)$ must be on the axes of $y$ and $z$ of the pitch quadric. We shall denote these screws by $\beta$ and $\gamma$, and their pitches $p_{\beta}$ and $p_{\gamma}$. From the properties of the cylindroid ( $\$ 15$ ) it appears that $a$, the semiaxis of the pitch quadric, must be determined from the equations-

$$
\begin{aligned}
& a=\left(p_{\beta}-p_{\gamma}\right) \sin l \cos l, \\
& p_{\beta} \cos ^{2} l+p_{\gamma} \sin ^{2} l=0 ;
\end{aligned}
$$

whence eliminating $l$, we deduce-

$$
a=\sqrt{-p_{\beta} p_{\gamma}} .
$$

If $b, c$ be the remaining semiaxes of the pitch quadric, then we must have-

$$
\frac{\cos ^{2} l}{b^{2}}+\frac{\sin ^{2} l}{c^{2}}=0
$$

because the screws $\mu, \nu$ are parallel to the asymptotes of

$$
\frac{y^{2}}{b^{2}}+\frac{z^{2}}{c^{2}}=1
$$

whence we find-

$$
p_{\beta^{2}}{ }^{2}=-\frac{a^{2} b^{2} c^{2}}{b^{4}}: p_{\gamma^{2}}{ }^{2}=-\frac{a^{2} b^{2} c^{2}}{c^{4}} .
$$


By taking the tangent planes to the pitch quadric at the extremities of $y$, we should similarly find-

$$
p_{a^{2}}=-\frac{a^{2} b^{2} c^{2}}{a^{4}}: p_{\gamma}{ }^{2}=-\frac{a^{2} b^{2} c^{2}}{c^{4}} ;
$$

hence we deduce the very important result which may be thus stated:-

The three principal axes of the pitch quadric, when furnished with suitable pitches $p_{a}, p_{\beta}, p_{\gamma}$, constitute screres belonging to the screw complex of the third order, and the equation of the pitch quadric has the form-

$$
p_{a} x^{2}+p_{\beta} y^{2}+p_{\gamma} z^{2}+p_{a} p_{\beta} p_{\gamma}=0 .
$$

We can also show conversely that every screw $\theta$ of zero pitch, which belongs to the screw complex of the third order, must be one of the generators of the pitch quadric. For $\theta$ must be reciprocal to all the screws of zero pitch on the reciprocal system of generators of the pitch quadric; and since two screws of zero pitch cannot be reciprocal unless they intersect either at a finite or infinite distance, it follows that $\theta$ must intersect the pitch quadric in an infinite number of points, and must therefore be entirely contained thereon.

Let now $S$ denote a screw complex of the third order, where $a, \beta, \gamma$ are the three screws of the system on the principal axes of the pitch quadric. Diminish the pitches of all the screws of $S$ by any magnitude $k$. Then the quadric-

$$
\begin{aligned}
\left(p_{a}-k\right) x^{2}+\left(p_{\beta}-k\right) & y^{2}+\left(p_{\gamma}-k\right) z^{2} \\
& +\left(p_{a}-k\right)\left(p_{\beta}-k\right)\left(p_{\gamma}-k\right)=0 \ldots \ldots
\end{aligned}
$$

must be the locus of screws of zero pitch in the altered system, and therefore of pitch $+k$ in the original system (§80). 
Regarding $k$ as a variable parameter, the equation just written represents the family of quadrics which constitute the screw complex $S$ and the reciprocal screw complex $S^{\prime}$. Thus all the generators of one system on each quadric, with pitch $+k$, constitute screws about which the body, with three degrees of freedom, can be twisted; while all the generators of the other system, with pitch $-k$, constitute screws, wrenches about which would be neutralized by the reaction of the constraints.

For the quadric to be a real surface it is plain that $k$ must be greater than the least, and less than the greatest of the three quantities $p_{a}, p_{\beta}, p_{\gamma}$. Hence the pitches of all the real screws of the screw complex $S$ are intermediate between the greatest and least of the three quantities $p_{a}, p_{\beta}, p_{\gamma}$.

§ I12. Screws through a Given Point.-We shall now show that three screws belonging to $S$, and also three screws belonging to $S^{\prime}$, can be drawn through any point $x^{\prime}, y^{\prime}, z^{\prime}$. Substitute $x^{\prime}, y^{\prime}, z^{\prime}$, in the equation of the last article, and we find a cubic for $k$. This shows that three quadrics of the system can be drawn through each point of space. The three tangent planes at the point each contain two generators, one belonging to $S$, and the other to $S^{\prime}$. It may be noticed that these three tangent planes intersect in a straight line.

Two intersecting screws can only be reciprocal if they be at right angles, or if the sum of their pitches be zero. It is hence easy to see that, if a sphere be described around any point as centre, the three screws belonging to $S$, which pass through the point, intersect the sphere in the vertices of a spherical triangle which is the polar of the triangle similarly formed by the lines belonging to $S^{\prime}$.

We shall now show that one screw belonging to $S$ 
can be found parallel to any given direction. All the generators of the quadric are parallel to the cone

$$
\left(p_{\alpha}-k\right) x^{2}+\left(p_{\beta}-k\right) y^{2}+\left(p_{\gamma}-k\right) z^{2}=0,
$$

and $k$ can be determined so that this cone shall have one generator parallel to the given direction; the quadric can then be drawn, on which two generators will be found parallel to the given direction; one of these belongs to $S$, while the other belongs to $S^{\prime}$.

It remains to be proved that each screw of $\mathrm{S}$ has a pitch which is proportional to the inverse square of the parallel diameter of the pitch quadric.*

Let $r$ be the intercept on a generator of the cone--

$$
\left(p_{a}-k\right) x^{2}+\left(p_{\beta}-k\right) y^{2}+\left(p_{\gamma}-k\right) z^{2}=0 ;
$$

by the pitch quadric-

$$
\begin{aligned}
& p_{a} x^{2}+p_{\beta} y^{2}+p_{\gamma} z^{2}+p_{a} p_{\beta} p_{\gamma}=0 ; \\
& \text { then } k=-\frac{p_{a} p_{\beta} p_{y}}{r^{2}} ;
\end{aligned}
$$

but $k$ is the pitch of the screw of $S$, which is parallel to the line $r$.

Nine constants $(\S 49)$ are required for the determination of a screw complex of the third order. This is the same number as that required for the specification of a quadric surface. $\dagger$ We hence infer, what is indeed other-

* This theorem is connected with some purely geometrical theorems of Plucher, who has shown (Neue Geometrie des Raumes, p. 130) that $k_{1} x^{2}$ $+k_{2} y^{2}+k^{3} z^{2}+k_{1} k_{2} k_{3}=0$, is the locus of lines common to three linear complexes of the first degree. The axes of the three complexes are directed along the co-ordinate axes, and the parameters of the complexes are $k_{1}, k_{2}, k_{3}$; the same author has also proved that the parameter of any complex belonging to the ("dreigliedrigen Gruppe") is proportional to the inverse square of the parallel diameter of the hyperboloid.

+ Salmon's Analytic Geometry of Three Dimensions, p. 35. 
wise manifest, viz., that when the pitch quadric is known the entire screw complex of the third order is determined.

Another very interesting property of the pitch quadric is thus enunciated. Any three co-reciprocal screws of a given screw complex of the third order are parallel to a triad of conjugate diameters of its pitch quadric.

Take any three co-reciprocal screws of the complex as screws of reference, and let $p_{1}, p_{2}, p_{3}$ be their pitches. If then the co-ordinates of any screw $\rho$ belonging to the complex be denoted by $\rho_{1}, \rho_{2}, \rho_{3}$, we shall have for the pitch of $\rho(\S 65)$ -

$$
p_{\rho}=p_{1} \rho_{1}{ }^{2}+p_{2} \rho_{2}{ }^{2}+p_{3} \rho_{3}{ }^{2} .
$$

If a parallelopiped be constructed, of which the three lines parallel to the reciprocal screws, drawn through the centre of the pitch quadric, are conterminous edges, and of which the line parallel to $\rho$ is the diagonal, and if $x, y, z$ be the lengths of the edges, and $r$ the length of the diagonal, then we have $(\$ 37)$ -

$$
\frac{x}{r}=\rho_{1}, \frac{y}{r}=\rho_{2}, \frac{z}{r}=\rho_{3} .
$$

It follows that $p_{p}$ must be proportional to the inverse square of the parallel diameter of the quadric surface-

$$
p_{1} x^{2}+p_{2} y^{2}+p_{3} z^{2}=H \text {. }
$$

But $p_{\rho}$ must be proportional to the inverse square of the parallel diameter of the pitch quadric, and hence the equation last written must actually be the equation of the pitch quadric, when $H$ is properly chosen. But the equation is obviously referred to three conjugate diameters, and hence three conjugate diameters of the pitch quadric are parallel to three co-reciprocal screws of the screw complex.

We see from this that the sum of the reciprocals of 
the pitches of three co-reciprocal screws is constant. This theorem will be subsequently generalised ( $\S 136)$.

$\S$ Ir 3. Screws of the Complex parallel to a Plane.-Up to the present we have been analysing the screw complex by classifying the screws into groups of constant pitch. Some interesting features will be presented by adopting a new method of classification. We shall now divide the general system into groups of screws which are parallel to the same plane.

We shall first prove that each of these groups constitutes a cylindroid. For suppose a screw of infinite pitch normal to the plane, 'then all the screws of the group parallel to the plane are reciprocal to this screw of infinite pitch. But they are also reciprocal to any three screws of the original reciprocal system ; they, therefore, form a screw complex of the second order $(\S 46)$-that is, they constitute a cylindroid.

We shall prove this in another manner.

A quadric containing a line must touch every plane passing through the line.* The number of screws of the complex which can lie in a given plane is, therefore, equal to the number of the quadrics of the complex which can be drawn to touch that plane.

The quadric surface whose equation is-

$$
\begin{aligned}
& \left(p_{\alpha}-k\right) z^{2}+\left(p_{\beta}-k\right) y^{2}+\left(p_{\gamma}-k\right) z^{2} \\
+ & \left(p_{\alpha}-k\right)\left(p_{\beta}-k\right)\left(p_{\gamma}-k\right)=0,
\end{aligned}
$$

touches the plane $P x+Q y+R z+S=0$, when the following condition is satisfied : $†-$

$$
\begin{aligned}
& p^{2}\left(p_{\beta}-k\right)\left(p_{\gamma}-k\right)+Q^{2}\left(p_{\alpha}-k\right)\left(p_{\gamma}-k\right) \\
+ & \left.R^{2}\left(p_{\alpha}-k\right) p_{\beta}-k\right)+S^{2}=0 ;
\end{aligned}
$$

* Salmon's Analytic Geometry of Three Dimensions, p. 74.

+ Salmon, loc. cit., p. 49. 
whence it follows that two values of $k$ can be found, or that two quadrics can be made to touch the plane, and that, therefore, two screws of the complex, and, of course, two reciprocal screws, lie in the plane.

From this it follows that all the screws of the complex parallel to a plane must lie upon a cylindroid. For, take any two screws parallel to the plane, and draw a cylindroid through these screws. Now, this cylindroid will be cut by any plane parallel to the given plane in two screws, which must belong to the complex; but this plane cannot contain any other screws; therefore, all the screws parallel to a given plane must lie upon the same cylindroid.

$\S$ I I4. Determination of the Cylindroid.-We now propose to solve the following problem:-Given a plane, determine the cylindroid which contains all the screws, selected from a screw complex of the third order, which are parallel to that plane.

Draw through $O$ the centre of the pitch quadric a plane $A$ parallel to the given plane. We shall first show that the centre of the cylindroid required lies in $A$.

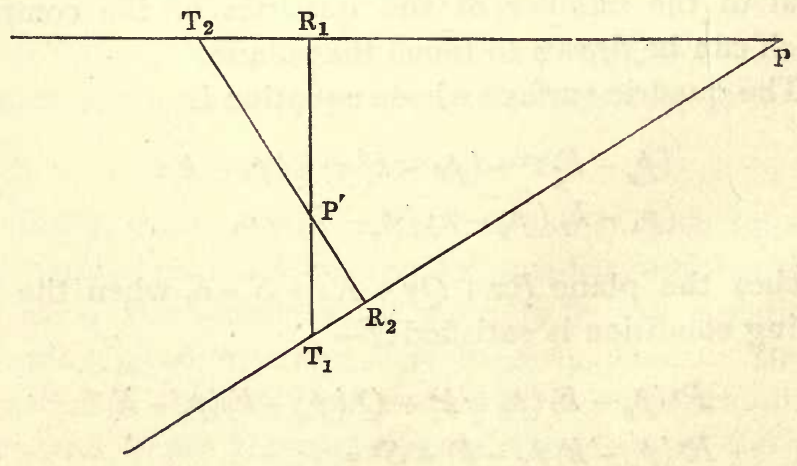

Fig. 3.

Let $T_{1}, T_{2}$ (Fig. 3) be two points in which the two quadrics of constant pitch touch the plane of the paper, 
which may be regarded as any plane parallel to $A$; then $P$ is the intersection of the pair of screws belonging to the complex $P T_{1}, P T_{2}$, which lie in that plane, and $P$ is the intersection of the pair of reciprocal screws $P^{\prime} R_{1}, P^{\prime} R_{2}$ belonging to the reciprocal complex. Since $P^{\prime} R_{1}$ is to be reciprocal to $P T_{2}$, it is essential that $R_{1}$ be a right angle, similarly $R_{2}$ is a right angle. The reciprocal cylindroid, whose axis passes through $P^{\prime}$, will be identically the same as the cylindroid belonging to the complex whose axis passes through $P$; but the two will be differently posited. If the angle at $P$ be a right angle, the points $T_{1}$ and $T_{2}$ are at infinity; therefore, the plane touches the quadric at infinity; it must, therefore, touch the asymptotic cone, and must, therefore, pass through the centre of the pitch quadric $O$; but $P$ is the centre of the cylindroid in this case, and, therefore, the centre of the cylindroid must lie in the plane $A$.

The position of the centre of the cylindroid in the plane $A$ is to be found by the following construction :Draw through the centre $O$ a diameter conjugate in the pitch quadric to the plane $A$. Let this line intersect the pitch quadric in the points $P_{1}, P_{2}$, and let $S, S^{\prime}$ (Fig. 4) be the feet of the perpendiculars let fall from $P_{1}, P_{2}$ upon the plane $A$. Draw the asymptotes $O L$, $O M$ to the section of the pitch quadric, made by the plane $A$. Through $S$ and $S^{\prime}$ draw lines in the plane $A$, $S T, S T^{\prime}, S^{\prime} T, S^{\prime} T^{\prime}$, parallel to the

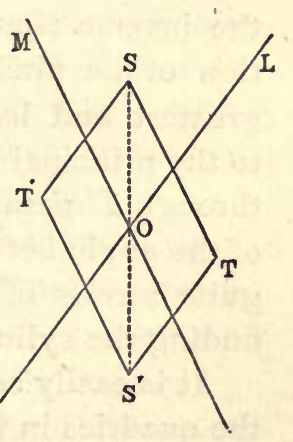

Fig. 4. asymptotes, then $T^{\prime}$ and $T$ are the centres of the two required cylindroids which belong to the two reciprocal screw complexes.

This construction is thus demonstrated :- 
The tangent planes at $P_{1}, P_{2}$ each intersect the surface in lines parallel to $O L, O M$. Let us call these lines $P_{1} L_{1}, P_{1}, M_{1}$ through the point $P_{1}$, and $P_{2} L_{2}, P_{2} M_{2}$ through the point $P_{2}$. Then $P_{1} L_{1}, P_{2} M_{2}$, are screws belonging to the complex, and $P_{1} M_{1}, P_{2} L_{2}$ are reciprocal screws.

Since $O L$ is a tangent to the pitch quadric, it therefore must be intersected by two rectilinear generators, one of each system. These two generators lie in a plane which contains $O L$; but since $O L$ touches the hyperboloid at infinity, the lines on the surface must be parallel to $O L$, and therefore their projections on the plane of $A$ must be $S^{\prime} T, S^{\prime} T^{\prime}$. Similarly for $S T, S^{\prime} T^{\prime}$; hence $S T^{\prime}$ and $S^{\prime} T^{\prime}$ are the projections of two screws belonging to the complex, and therefore the centre of the cylindroid is at $T^{\prime}$. In a similar way it is proved that the centre of the reciprocal cylindroid is at $T$.

Having thus determined the centre of the cylindroid, the remainder of the construction is easy. The pitches of two screws on the surface must be proportional to the inverse square of the parallel diameters of the section of the pitch quadric made by $A$. Therefore, the greatest and least pitches will be on screws parallel to the principal axes of the section. Hence, lines drawn through $T^{\prime}$ parallel to the external and internal bisectors. of the angle between the asymptotes are the two rectangular screws of the cylindroid. Thus the problem of finding the cylindroid is completely solved.

It is easily seen that each cylindroid touches each of the quadrics in two points.

$\S$ I1 5. Miscellaneous Remarks. - It follows from the last article that any plane which contains a pair of screws belonging to the complex which intersect at right angles must pass through the centre of the pitch quadric.

We are now in a position to determine the actual 
situation of a screw $\theta$ belonging to a screw complex of the third order of which the direction is given. The construction is as follows:-Draw through $O$ the centre of the pitch quadric a radius vector $O R$ parallel to the given direction of $\theta$, and cutting the pitch quadric in $R$. Draw a tangent plane to the pitch quadric in $R$. Then the plane $A$ through $O R$, of which the intersection with the tangent plane is perpendicular to $O R$, is the plane which contains $\theta$. For the section in which $A$ cuts the pitch quadric has for a tangent at $R$ a line perpendicular to $O R$; hence the line $O R$ is a principal axis of the section, and hence ( $\& I 14)$ one of the two screws of the complex in the plane $A$ must be parallel to $O R$. It remains to find the actual situation of $\theta$ in the plane $A$.

Since the direction of $\theta$ is known, its pitch is determinate, because it is inversely proportional to the square of $O R$. Hence the quadric can be constructed, which is the locus of all the screws which have the same pitch as $\theta$. This quadric must be intersected by the plane $A$ in two parallel lines. One of these lines is the required residence of the screw $\theta$, while the other line, with a pitch equal in magnitude to that of $\theta$, but opposite in sign, belonging, as it does, to one of the other system of generators, is a screw reciprocal to the system.

A family of quadric surfaces of constant pitch have the same planes of circular section, and therefore every plane through the centre cuts the quadrics in a system of conics having the same directions of axes.

The cylindroid which contains all the screws of the screw complex parallel to one of the planes of circular section must be composed of screws of equal pitch. A cylindroid in this case reduces to a plane pencil of rays passing through a point. We thus have two points situated upon the primary axis of the pitch quadric, 
through each of which a plane pencil of screws can be drawn, which belong to the screw complex. All the screws passing through either of these points have equal pitch. The pitches of the two pencils are equal in magnitude, but opposite in sign. The magnitude is that of the pitch of the screw situated on the primary axis of the pitch quadric.*

§ I 16. Virtual co-efficients.-Let $\rho$ be a screw of the screw complex which makes angles whose cosines are $f, g, h$, with the three screws of reference $a, \beta, \gamma$ upon the axes of the pitch quadric. Then, reference being made to any six co-reciprocals, we have for the coordinates of $\rho$,

$$
\begin{gathered}
\rho_{1}=f a_{1}+g \beta_{1}+h \gamma_{1}, \\
\& c ., \& c ., \\
\rho_{6}=f a_{6}+g \beta_{6}+h \gamma_{6} .
\end{gathered}
$$

Let $\eta$ be any given screw. The virtual co-efficient of $p$ and $\eta$ is-

$$
2 f \varpi_{\sigma_{\eta}}+2 g \varpi_{\beta_{\eta}}+2 h \omega_{\eta_{\eta}} \text {. }
$$

Draw from the centre of the pitch quadric a radius vector $r$ parallel to $\rho$, and equal to the virtual coefficient just written; then the locus of the extremity of $r$ is the sphere-

$$
x^{2}+y^{2}+z^{2}=2\left[x \overline{w a \eta}+y \bar{w}_{\beta \eta}+z \varpi^{m}\right] \text {. }
$$

The tangent plane to the sphere obtained by equating the right-hand side of this equation to zero is the prin-

* If $a, b, c$ be the three semiaxes of the pitch quadric, and $+d$ the distances from the centre, on $a$, of the two points in question, it appears from $\S$ I I 4 that $a^{2} d^{2}=\left(a^{2}-b^{2}\right)\left(a^{2}-c^{2}\right)$, which shows that $d$ is the fourth proportional to the primary semiaxis of the surface, and of its focal ellipse and hyperbola. 
cipal plane of that cylindroid which contains all the screws of the screw complex which are reciprocal to $\eta$.

$\S$ II7. Four Screws of the Screw Complex.-Take any four screws $a, \beta, \gamma, \delta$ of the screw complex of the third order. Then we shall prove that the cylindroid $(a, \beta)$ must have a screw in common with the cylindroid $(\gamma, \delta)$ For twists of appropriate amplitudes about $a, \beta, \gamma, \delta$ must neutralise, and hence the twists about $a, \beta$ must be counteracted by those about $\gamma, \delta$; but this cannot be the case unless there is some screw common to $(a, \beta)$ and $(\gamma, \delta)$.

This theorem provides a convenient test as to whether four screws belong to a screw complex of the third order.

\& I 1 8. Equilibrium of Four Forces applied to a Rigid Body.If the body be free, the four forces must be four wrenches on screws of zero pitch which are members of a screw complex of the third order. The forces must therefore be generators of an hyperboloid, and all belonging to the same sysiem ( $\S 106)$.

Three of the forces, $P, Q, R$, being given in position, $S$ must then be a generator of the hyperboloid determined by $P, Q, R$. This proof of a well-known theorem (due to Möbius) is given to show the facility with which such results flow from the Theory of Screws.

Suppose, however, that the body have only freedom of the fifth order, we shall find that somewhat more latitude exists with reference to the choice of $S$. Let $X$ be the screw reciprocal to the screw complex by which the freedom is defined. Then for equilibrium it will only be necessary that $S$ belong to the complex of the fourth order defined by the four screws

$$
P, Q, R, X \text {. }
$$

A cone of screws can be drawn through eevry point K 2 
in space belonging to this complex, and on that cone one screw of zero pitch can always be found. Hence one line can be drawn through every point in space along which $S$ might act.

If the body have freedom of the fourth order, the latitude in the choice of $S$ is still greater. Let $X_{1}, X_{2}$ be two screws reciprocal to the complex, then $S$ is only restrained by the condition that it belong to the screw complex of the fifth order defined by the screws

$$
P, Q, R, X_{1}, X_{2} \text {. }
$$

Any line in space when it receives the proper pitch is a screw of this complex. Through any point in space a plane can be drawn such that every line in the plane passing through the point with zero pitch is a screw of the complex ( $\$ 80)$.

Finally, if the body has only freedom of the third order, the four equilibrating forces $P, Q, R, S$ may be situated anywhere.

The positions of the forces being given, their magnitudes are determined; for draw three screws $X_{1}, X_{2}, X_{3}$ reciprocal to the complex, and find $(\S 30)$ the intensities of the seven equilibrating wrenches on

$$
P, Q, R, S, X_{1}, X_{2}, X_{3} \text {. }
$$

The last three are neutralised by the reactions of the constraints, and the four former must therefore equilibrate.

Given any four screws in space, it is possible for four wrenches of proper intensilies on these screws to hold a body having freedom of the third order in equilibrium. For, take the four given screws, and three reciprocal screws. Wrenches of proper intensities on these seven screws will equilibrate; but those on the reciprocal screws 
are destroyed by the reactions, and, therefore, the four wrenches on the four screws equilibrate. It is manifest that this theorem may be generalised into the following:-If a body have freedom of the $k^{\text {th }}$ order, then properly selected wrenches about any $k+$ I screws (not reciprocal to the screw complex) will hold the body in equilibrium.

That a rigid body with freedom of the third order may be in equilibrium under the action of gravity, we have the necessary and sufficient condition, which is thus stated :-

The vertical through the centre of inertia must be one of the reciprocal system of generators on the pitch quadric.

We see that the centre of inertia must, therefore, lie upon a screw of zero pitch which belongs to the screw complex; whence we have the following theorem :The restraints which are necessary for the equilibrium of a body which has freedom of the third order under the action of gravity, would permit rotation of the body round one definite line through the centre of inertia.

$\S$ II9. The Ellipsoid of Inertia.-The momental ellipsoid, which is of such significance in the theory of the rotation of a rigid body about a fixed point, is presented in the Theory of Screws as a particular case of another ellipsoid called the ellipsoid of inertia, which is of great importance in connexion with the general screw complex of the third order.

If we take three conjugate screws of inertia from the screw complex, as screws of reference, then we have seen $\left(\$ \sigma_{7}\right)$ that, if $\theta_{1}, \theta_{2}, \theta_{3}$, be the co-ordinates of a screw $\theta$, we have-

$$
u_{\theta}^{2}=u_{1}^{2} \theta_{1}^{2}+u_{2}^{2} \theta_{2}^{2}+u_{3}^{2} \theta_{3}^{2}
$$

where $u_{1}, u_{2}, u_{3}$ are the values of $u_{\theta}$ with reference to the three conjugate screws of inertia. 
Draw from any point lines parallel to $\theta$, and to the three conjugate screws of inertia. If then a parallelopiped be constructed of which the diagonal is the line parallel to $\theta$, and of which the three lines parallel to the conjugate screws are conterminous edges, and if $r$ be the length of the diagonal, and $x, y, z$ the lengths of the edges, then we have-

$$
\frac{x}{r}=\theta_{1}, \frac{y}{r}=\theta_{2}, \frac{z}{r}=\theta_{3} .
$$

We see, therefore ${ }_{1}$ that the parameter $u$ appropriate to any screw $\theta$ is inversely proportional to the parallel diameter of the ellipsoid

$$
u_{1}^{2} x^{2}+u_{2}^{2} y^{2}+u_{3}^{2} z^{2}=H .
$$

where $H$ is a certain constant.

Hence we have the following theorem :- The kinetic energy of a rigid body, when twisting with a given twist velocity about any screw of a complex of the third order, is proportional to the inverse square of the parallel diameter of a certain ellipsoid, which may be called the ellipsoid of inertia; and a set of three conjugate diameters of the ellipsoid are parallel to a set of three conjugate screws of inertia which belong to the screw complex.

We might also enunciate the property in the following manner :-Any diameter of the ellipsoid of inertia is proportional to the twist velocity with which the body should twist about the parallel screw of the screw complex, so that its kinetic energy shall be constant.

$\S$ I 20. The Principal Screws of Inertia.-It will simplify matters to consider that the ellipsoid of inertia is concentric with the pitch quadric. It will then be possible to find a triad of common conjugate diameters to the two ellipsoids. We can then determine three screws of the complex parallel to these diameters ( $\$$ II5), 
and these three screws will be co-reciprocal, and also conjugate screws of inertia. They will, therefore ( $\$ 57)$, form what we have termed the principal screws of inertia. When the screw complex reduces to a pencil of screws of zero pitch passing through a point, then the principal screws of inertia reduce to the well-known principal axes.

§ I2I. Lemma.-If from a screw complex of the $n^{\text {th }}$ order we select $n$ screws $A_{1}, \ldots, A_{n}$, which are conjugate screws of inertia ( $\left(57\right.$ ), and if $S_{1}$ be any screw which is reciprocal to $A_{2}, \ldots, A_{n}$, then an impulsive wrench on $S_{1}$ will cause the body, when only free to twist about the screws of the complex, to commence to twist about $A_{1}$. Let $R_{1}$ be the screw which, if the body were perfectly free, would be the impulsive screw corresponding to $A_{1}$ as the instantaneous screw. $R_{1}$ must be reciprocal to $A_{2}, \ldots, A_{n}(\S 54)$. Take also $6-n$ screws of the reciprocal system $B_{1}, \ldots, B_{6-n}$. Then the $8-n$ screws $R_{1}, S_{1}, B_{1}, \ldots, B_{6-n}$ must be reciprocal to the $n-1$ screws $A_{2}, \ldots A_{n}$, and therefore the $8-n$ screws must belong to a screw complex of the $(7-n)^{\text {th }}$ order. Hence an impulsive wrench upon the screw $S_{1}$ can be resolved into components on $R_{1}, B_{1}, \ldots B_{6} n$. Of these all but the first are neutralised by the reactions of the constraints, and by hypothesis the effect of an impulsive wrench upon $R_{1}$ is to make the body commence to twist about $A_{1}$, and therefore an impulsive wrench on $S_{1}$ would make the body twist about $A_{1}$.

§ 122. Relation between the Impulsive Screw and the Instantaneous Screw.-A quiescent rigid body which possesses freedom of the third order is acted upon by an impulsive wrench about a given screw $\eta$. It is required to determine the instantaneous screw $\theta$, about which the body will commence to twist.

The screws which belong to the complex, and are at 
the same time reciprocal to $\eta$, must all lie upon a cylindroid, as they each fulfil the condition of being reciprocal to four screws. All the screws on the cylindroid are parallel to a certain plane drawn through the centre of the pitch quadric, which may be termed the reciprocal plane with respect to the screw $\eta$. The reciprocal plane having been found, the diameter conjugate to this plane in the ellipsoid of inertia is parallel to the required screw $\theta$.

For let $\mu$ and $\nu$ denote two screws of the complex parallel to a pair of conjugate diameters of the ellipsoid of inertia in the reciprocal plane. Then $\theta, \mu, \nu$ are a triad of conjugate screws of inertia; but $\boldsymbol{\eta}$ is reciprocal to $\mu$ and $\nu$, and, therefore, by the lemma of the last article, an impulsive wrench upon $\eta$ will make the body commence to twist about $\theta$.

$\S$ 123. Kinetic acqEnergy uired by an Impulse.-We shall now consider the following problem:-A quiescent rigid body of mass $M$ receives an impulsive wrench of intensity $\eta^{\prime \prime}$ on a screw $\eta$ for a short time $e$. Determine the locus of a screw $\theta$ belonging to a screw complex of the third order, such that, if the body be constrained to twist about $\theta$, it shall acquire a given kinetic energy $K$, in consequence of the impulsive wrench.

We have from $\$ 6 \mathrm{r}$ the equation-

$$
K=\frac{e^{2}}{M} \frac{\eta^{\prime \prime 2}}{u_{\theta}^{2}} \varpi_{\eta \theta}^{2} .
$$

We can assign a geometrical interpretation to this equation, which will lead to some interesting results.

Through the centre $O$ of the pitch quadric the plane $A$ reciprocal to $\eta$ is to be drawn. A sphere (\$116) is to be described touching the plane $A$ at the origin $O$, the diameter of the sphere being so chosen that the intercept $O P$ made by the sphere on a radius vector 
parallel to any screw $\theta$ is equal to $\varpi_{\eta \theta}(\S I I 6)$. The quantity $u_{\theta}$ is inversely proportional to the radius vector $O Q$ of the ellipsoid of inertia, which is parallel to $\theta$ ( $I$ I 9 ). Hence for all the screws of the screw complex which acquire a given kinetic energy in consequence of a given impulse, we must have the product $O P . O Q$ constant.

From a well-known property of the sphere, it follows that all the points $Q$ must lie upon a plane $A^{\prime}$, parallel to $A$. This plane cuts the ellipsoid of inertia in an ellipse, and all the screws required must be parallel to the generators of the cone of the second degree, formed by joining the points of this ellipse to the origin, $O$.

Since we have already shown how, when the direction of a screw belonging to a screw complex of the third order is given, the actual situation of that screw is determined (§ I I 5), we are now enabled to ascertain all the screws $\theta$ on which the body acted upon by a given impulse would acquire a given kinetic energy.

The distance between the planes $A$ and $A^{\prime}$ is proportional to $O P . O Q$, and therefore to the square root of $K$. Hence, when the impulse is given, the kinetic energy acquired on a screw determined by this construction is greatest when $A$ and $A^{\prime}$ are as remote as possible. For this to happen, it is obvious that $A^{\prime}$ will just touch the ellipsoid of inertia. The group of screws will, therefore, degenerate to the single screw parallel to the diameter of the ellipsoid of inertia conjugate to $A$. But we have seen ( $\$ 122)$ that the screw so determined is the screw which the body will naturally select if permitted to make a choice from all the screws of the complex of the third order. We thus see again what Euler's theorem $(\$ 64)$ would have also told us, viz., that when a quiescent rigid body which has freedom of the third order is 
set in motion by the action of an impulsive wrench, the kinetic energy which the body acquires is greater than it would have been had the body been restricted to any other screw of the complex than that one which it naturally chooses.

\& 124. Reaction of the Constraints.-An impulsive wrench on a screw $\eta$ acts upon a body with freedom of the third order, and the body commences to move by twisting upon a screw $\theta$. It is required to find the screw $\lambda$, a wrench on which constitutes the initial reaction of the constraints. Let $\phi$ denote the impulsive screw which, if the body were free, would correspond to $\theta$ as the instantaneous screw. Then $\lambda$ must lie upon the cylindroid $(\phi, \eta)$, and may be determined by choosing on $(\phi, \eta)$ a screw reciprocal to any screw of the given screw complex.

$\S 125$. Impulsive Screw is Indeterminate.-Being given the instantaneous screw $\theta$ in a complex of the third order, the corresponding impulsive screw $\eta$ is indeterminate, because the impulsive wrench may be compounded with any reactions of the constraints. In fact $\eta$ may be any screw selected from a screw complex of the fourth order, which is thus found. Draw the diametral plane conjugate to a line parallel to $\theta$ in the ellipsoid of inertia, and construct the cylindroid which consists of screws belonging to the screw complex parallel to this diametral plane. Then any screw which is reciprocal to this cylindroid will be an impulsive screw corresponding to $\theta$ as an instantaneous screw.

Thus we see that through any point in space a whole cone of screws can be drawn, an impulsive wrench on any one of which would make the body commence to twist about the same screw.

One impulsive couple can always be found which would make the body commence to twist about any 
given screw of the screw complex. For a couple in a plane perpendicular to the nodal line of a cylindroid may be regarded as a wrench upon a screw reciprocal to the cylindroid; and hence a couple in a diametral plane of the ellipsoid of inertia, conjugate to the diameter parallel to the screw $\theta$, will make the body commence to twist about the screw $\theta$.

It is somewhat remarkable that a force directed along the nodal line of the cylindroid must make the body commence to twist about precisely the same screw as the couple in a plane perpendicular to the nodal line.

If a cylindroid be drawn through two of the principal screws of inertia, then an impulsive wrench on any screw of this cylindroid will make the body commence to twist about a screw on the same cylindroid. For the impulsive wrench may be resolved into wrenches on the two principal screws. Each of these will produce a twisting motion about the same screw, which will, of course, compound into a twisting motion about a screw on the same cylindroid.

$\S$ I 26. Ellipsoid of the Potential.-A body which has freedom of the third order is in equilibrium under the influence of a system of forces in conformity with the restrictions of $\S 6$. The body receives a twist of small amplitude $\theta^{\prime}$ about a screw $\theta$ of the screw complex. It is required to determine a geometrical representation for the quantity of work which has been done in effecting the displacement. We have seen that to each screw $\theta$ corresponds a certain linear parameter $v_{\theta}(\S 72)$, and that the work done is represented by-

\section{$F v \sigma^{2} \theta^{\prime 2}$.}

We have also seen that the quantity $v_{\theta}^{2}$ may be represented by-

$$
v_{1}^{2} \theta_{1}^{2}+v_{2}^{2} \theta_{2}^{2}+v_{3} \theta_{3}^{2} ;
$$


where $\theta_{1}, \theta_{2}, \theta_{3}$ are the co-ordinates of the screw $\theta$ referred to three conjugate screws of the potential, and $v_{1}, v_{2}, v_{3}$, denote the values of $v_{\theta}$ for each of the three screws of reference $(\S 72)$.

Drawing through the centre of the pitch quadric three axes parallel to the three screws of reference, we can then construct the ellipsoid of which the equation is-

$$
v_{1}^{2} x^{2}+v_{2}^{2} y^{2}+v_{3}^{2} z^{2}=H \text {, }
$$

which proves the following theorem.

The work done in giving the body a twist of given amplitude from a position of equilibrium about any screw of a complex of the third order, is proportional to the inverse square of the parallel diameter of a certain ellipsoid which we may call the ellipsoid of the potential, and three conjugate diameters of this ellipsoid are parallel to three conjugate screws of the potential in the screw complex.

\$ 127. The Principal Screws of the Potential.-The three common conjugate diameters of the pitch hyperboloid, and the ellipsoid of the potential, are parallel to three screws of the complex which are what we call the principal screws of the potential. If the body be displaced by a twist about a principal screw of the potential from a position of stable equilibrium, then the reduced wrench which is evoked is upon the same screw.

The three principal screws of the potential must not be confounded with the three screws of the complex which are parallel to the principal axes of the ellipsoid of the potential. The latter are the screws on which a twist of given amplitude requires a maximum or minimum consumption of energy, and they are rectangular, which, of course, is not in general the case with the principal screws of the potential.

$\S 128$. Wrench evoked by Displacement.-By the aid of 
the ellipsoid of the potential we shall be able to solve the problem of the determination of the screw on which a wrench is evoked by a twist about a given screw $\theta$ of the complex. The construction which will now be given will enable us to determine the screw of the complex on which the reduced wrench acts.

Draw through the centre of the pitch quadric a line parallel to $\theta$. Construct the diametral plane $A$ of the ellipsoid of the potential conjugate to this line, and let $\lambda, \mu$ be any two screws of the complex parallel to a pair of conjugate diameters of the ellipsoid of the potential which lie in the plane $A$. Then the required screw $\phi$ is parallel to that diameter of the pitch quadric which is conjugate to the plane $A$.

For $\phi$ will then be reciprocal to both $\lambda$ and $\mu$; and as $\lambda, \mu, \theta$ are conjugate screws of the potential, it follows that a twist about $\theta$ must evoke a reduced wrench on $\phi$.

§ 129. Harmonic Screws.-When a rigid body has free dom of the third order, it must have ( $\$ 74)$ three harmonic screws, or screws which are conjugate screws of inertia as well as conjugate screws of the potential. We are now enabled to construct these screws with facility, for they must be those screws of the screw complex which are parallel to the triad of common conjugate diameters of the ellipsoid of inertia, and the ellipsoid of the potential.

We have thus a complete geometrical conception of the small oscillations of a rigid body which has freedom of the third order. If the body be once set twisting about one of the harmonic screws, it will continue to twist thereon for ever, and in general its motion will be compounded of twisting motions upon the three harmonic screws.

If the displacement of the body from its position of 
equilibrium has been effected by a small twist about a screw on the cylindroid which contains two of the harmonic screws, then the twist can be decomposed into components on the harmonic screws, and the instantaneous screw about which the body is twisting at any epoch will oscillate backwards and forwards upon the cylindroid, from which it will never depart.

If the periods of the twist oscillations on two of the harmonic screws coincided, then every screw on the cylindroid which contains those harmonic screws would also be a harmonic screw.

If the periods of the three harmonic screws were equal, then every screw of the complex would be a harmonic screw.

$\S$ I 30. Oscillations of a Rigid Body about a Fixed Point.We shall conclude the present Chapter by applying the principles which it contains to the development of a geometrical solution of the following important problem :-

A rigid body, free to rotate in every direction around a fixed point, is at rest under the infuence of gravity. The body is slightly disturbed : it is required to determine the nature of its small oscillations.

Since three co-ordinates are required to specify the position of a body when rotating about a point, it follows that the body has freedom of the third order. The screw complex, however, assumes a very extreme type, for the pitch quadric has become illusory, and the screw complex reduces to a pencil of screws of zero pitch radiating in all directions from the fixed point.

The quantity $u_{\theta}$ appropriate to a screw $\theta$ reduces to the radius of gyration when the pitch of the screw is zero; hence the ellipsoid of inertia reduces in the present case to the well-known momental ellipsoid.

The ellipsoid of the potential $(\S 126)$ assumes a 
remarkable form in the present case. The work done in giving the body a small twist is proportional to the vertical distance through which the centre of inertia is elevated. Now, as in the position of equilibrium the centre of inertia is vertically beneath the point of suspension, it is obvious from symmetry that the ellipsoid of the potential must be a surface of revolution about a vertical axis. It is further evident that the vertical radius vector of the ellipsoid must be infinite, because no work is done in rotating the body around a vertical axis.

Let $O$ be the centre of suspension, and $I$ the centre of inertia, and let $O P$ be a radius vector of the ellipsoid of the potential. Let fall $I Q$ perpendicular on $O P$, and $P T$ perpendicular upon $O I$. It is extremely easy to show that the vertical height through which $I$ is raised is proportional to $I Q^{2} \times O P^{2}$; whence the area of the triangle $O P I$ is constant, and therefore the locus of $P$ must be a right circular cylinder of which $O I$ is the axis.

We have now to find the triad of common conjugate diameters of the momental ellipsoid, and the circular cylinder just described. A group of three conjugate diameters of the cylinder must consist of the vertical axis, and any two other lines through the origin, which are conjugate diameters of the ellipse in which their plane cuts the cylinder. It follows that the triad required will consist of the vertical axis, and of the pair of common conjugate diameters of the two ellipses in which the plane conjugate to the vertical axis in the momental ellipsoid cuts the momental ellipsoid and the cylinder. These three lines are the three harmonic axes.

With reference to the vertical axis which appears to be one of the harmonic axes, the time of vibration would 
be infinite, so we reject it. The three harmonic screws which are usually found in the small oscillations of a body with freedom of the third order are therefore reduced in the present case to two, and we have the following theorem:-

A rigid body which is free to rotate about a fixed point is at rest under the action of gravity. If a plane $\mathrm{S}$ be drawn through the point of suspension $\mathrm{O}$, congate to the vertical diameter OI of the momental ellipsoid, then the common conjugate diameters of the trwo ellipses in which $\mathrm{S}$ cuts the momental ellipsoid, and a circular cylinder whose axis is OI, are the two harmonic axes. If the body be displaced by a small rotation about one of these axes, the body will continue for ever to oscillate to and fro upon this axis, just as if the body had been actually constrained to move about this axis.

To complete the solution for any initial circumstances of the rigid body, a few additional remarks are necessary.

Assuming the body in any given position of equilibrium, it is first to be displaced by a small rotation about an axis $O X$. Draw the plane containing $O I$ and $O X$, and let it cut the plane $S$ in the line $O Y$. The small rotation around $O X$ may be produced by a small rotation about $O I$, followed by a small rotation about $O Y$. The effect of the small rotation about $O I$ is merely to alter the azimuth of the position, but not to disturb the equilibrium. Had we chosen this altered position as that position of equilibrium from which we started, the initial displacement will be communicated by a rotation around $O Y$. We may, therefore, without any sacrifice of generality, assume that the axis about which the initial displacement is imparted lies in the plane $S$. We shall now suppose the body to receive a small angular 
velocity about any other axis. This axis must be in the plane $S$, if small oscillations are to exist at all, for the initial angular velocity, if not capable of being resolved completely on the two harmonic axes, will have component around the vertical axis $O I$. The effect of an initial rotation about $O I$ will be to give the body a continuous slow rotation around the vertical axis, which is, of course, not admissible when small oscillations only are considered.

If, therefore, the body performs small oscillations only, we may regard the initial axis of displacement as lying in the plane $S$, while we must have the initial instantaneous axis in that plane. The initial displacement may be resolved into two displacements, one on each of the harmonic axes, and the initial angular velocity may also be resolved into two angular velocities on the two harmonic axes. The entire motion will, therefore, be found by compounding the vibrations about the two harmonic axes. Also the instantaneous axis will at every instant be found in the plane of the harmonic axes, and will oscillate to and fro in their plane.

Since conjugate diameters of an ellipse are always projected into conjugate diameters of the projected ellipse, it follows that the harmonic axes must project into two conjugate diameters of a circle on any horizontal plane. Hence we see that two vertical planes, each containing one of the harmonic axes, are at right angles to each other.

We have thus obtained a complete solution of the problem of the small oscillations of a body about a fixed point under the influence of gravity. 


\section{( 146$)$}

\section{CHAPTER XII.}

THE DYNAMICS OF A RIGID BODY WHICH HAS FREEDOM OF THE FOURTH ORDER.

$\S$ I3I. Screw Complex of the Fourth Order.-The most general type of a screw complex of the fourth order is merely a group of screws which are reciprocal to an arbitrary cylindroid $(\$ 49)$. To obtain a clear idea of this screw complex it is, therefore, only required to re-state a few results already obtained.

All the screws belonging to a screw complex of the fourth order which can be drawn through a given point lie on a cone of the second degree ( $\$ 25)$.

All the screws of given pitch belonging to a screw complex of the fourth order must intersect two fixed lines, viz., the two screws on the reciprocal cylindroid of pitch equal in magnitude, and opposite in sign, to the given pitch ( $\$ 24)$.

One screw of given pitch belonging to a screw complex of the fourth order can be drawn through each point in space ( $\$ 97)$.

$\S$ I 32. Screws Parallel to a Given Line.-It is required to determine the locus of the screws parallel to a given straight line $L$, which belong to a screw complex of the fourth order. This easily appears from the principle that each screw of the screw complex must intersect one screw of the reciprocal cylindroid at right angles (\$24). Take, therefore, that one screw $\theta$ on the cylindroid which is perpendicular to $L$. Then a plane through $\theta$ parallel to $L$ is the required locus.

$\S$ I33. Screws in a Plane.-As we have already seen that two screws belonging to a screw complex of the third order can be found in any plane ( $\$$ II $\left._{3}\right)$, so we might 
expect to find that a singly infinite number of screws belonging to a screw complex of the fourth order can be found in any plane. We shall now prove that all these screws envelope a parabola.

Take any point $P$ in the plane, then the screws through $P$ reciprocal to the cylindroid form a cone of the second order, which is cut by the plane in two lines. Thus two screws belonging to a given screw complex of the fourth order can be drawn in a given plane through a given point. From the last article it follows that only one screw of the complex parallel to a given line can be found in the plane. Therefore, the envelope must be a parabola.

§.I34. Property of the Pitches of Six Co-reciprocals.We may here introduce an important property of the pitches of a set of co-reciprocal screws selected from a screw complex.

There is one screw on a cylindroid of which the pitch is a maximum, and another screw of which the pitch is .a minimum. These screws are parallel to the principal axes of the pitch conic ( $\S 20)$. Belonging to a screw complex of the third order we have, in like manner, three screws of maximum or miminum pitch, which lie along the three principal axes of the pitch quadric ( I I I). The general question, therefore, arises, as to whether it is always possible to select from a screw complex of the $n^{\text {th }}$ order a certain number of screws of maximum or minimum pitch.

Let $\theta_{1}, \ldots \theta_{n}$ be the $n$ co-ordinates of a screw re ferred to $n$ co-reciprocal screws belonging to the given screw complex. Then the function $p_{\theta}$, or

$$
p_{1} \theta_{1}^{2}+\ldots+p_{n} \theta_{n}^{2}
$$

is to be a maximum, while, at the same time, the co-ordinates satisfy the condition ( $\$ 37$ )- 


$$
\Sigma \theta_{1}^{2}+2 \Sigma \theta_{1} \theta_{2} \cos \left(\omega_{1}, \omega_{2}\right)=\mathrm{I},
$$

which for brevity we denote as heretofore by-

$$
R=\mathbf{I} \text {. }
$$

Applying the ordinary rules* for maxima and minima, we deduce the $n$ equations-

$$
\begin{gathered}
2 p_{1} \theta_{1}-p_{\theta} \frac{d R}{d \theta_{1}}=0, \\
\& c . \& c_{.}, \\
2 p_{n} \theta_{n}-p_{\theta} \frac{d R}{d \theta_{n}}=0 .
\end{gathered}
$$

From these $n$ linear equations it would seem that $\theta_{1}, \ldots, \theta_{n}$ can be eliminated, and that an algebraic equation of the $n^{\text {th }}$ degree would remain for $p_{\theta}$. The analysis would, therefore, appear to have proved that $n$ screws of maximum or minimum pitch can always be selected from a screw complex of the $n^{\text {th }}$ order.

A moment's reflection will, however, show that this statement needs modification. Take the case of $n=6:$ the screw complex of the sixth order is simply another name for every screw in space. In this case, therefore, all the values of $p_{\theta}$ must be infinite, which implies that each co-efficient of the equation for $p_{\theta}$ must vanish, except the absolute term.

We are thus presented with no fewer than six formulæ involving the pitches and angles of inclination of the six screws of a co-reciprocal system. Of these formulæ we shall in this place only consider one. If the co-efficient of $p_{\theta}$ be equated to zero it appears that-

$$
\frac{\mathbf{I}}{p_{1}}+\frac{\mathbf{I}}{p_{2}}+\frac{\mathbf{I}}{p_{3}}+\frac{\mathbf{I}}{p_{4}}+\frac{\mathbf{I}}{p_{5}}+\frac{\mathbf{I}}{p_{6}}=0,
$$

* Williamson's Differential Calculus, 2nd Edition, p. I89. 
or, the sum of the reciprocals of the pitches of the six screws of a co-reciprocal system is equal to zero.

\$ 135. Another Proof.-The following elegant proof of the theorem of the last section was communicated to me by my friend Professor Everett. Divide, the six co-reciprocals into any two groups $A$ and $B$ of three each, then it appears from $\S$ I I I that the pitch quadric of each of these groups is identical. The three screws of $A$ are parallel to a triad of conjugate diameters of the pitch quadric, and the sum of the reciprocals of the pitches is proportional to the sum of the squares of the conjugate diameters ( $\S \mathrm{II} 2$ ). The three screws of $B$ are parallel to another triad of conjugate diameters of the pitch quadric, and the sum of the reciprocals of the pitches, with their signs changed, is proportional to the sum of the squares of the conjugate diameters. Remembering that the sum of the squares of the two sets of conjugate diameters is equal, the required theorem is at once evident.

$\S$ I 36. Property of the Pitches of $n$ Co-reciprocals. - The theorem just proved can be extended to show that the sum of the reciprocals of the pitches of $\mathrm{n}$ co-reciprocal screws, selected from a screw complex of the $\mathrm{n}^{\text {th }}$ order, is a constant for each screw complex.

Let $A$ be the given screw complex, and $B$ the reciprocal screw complex. Take $6-n$ co-reciprocal screws on $B$, and any $n$ co-reciprocal screw on $A$. The sum of the reciprocals of the pitches of these six screws must be always zero; but the screws on $B$ may be constant, while those on $A$ are changed, whence the sum of the reciprocals of the pitches of the $n$ co-reciprocal screws on $A$ must be constant.

Thus, as we have already seen from geometrical considerations, that the sum of the reciprocals of the pitches of co-reciprocals is constant for the screw complex of 
the second and third order $(\$ \$ 2,112)$, so now we see that the same must be likewise true for the fourth, fifth, and sixth orders.

The actual value of this constant for any given screw complex is evidently a characteristic feature of that screw complex.

$\S$ 137. Special Screw of the Complex.-In general there is one line in each csrew complex of the fourth order, which forms a screw belonging to the screw complex, whatever be the pitch assigned to it. The line in question is the nodal line of the reciprocal cylindroid. The kinematical statement is as follows :-

When a rigid body has freedom of the fourth order, there is in general one straight line, about which the body can be rotated, and parallel to which it can be translated.

$\S$ 138. Particular Case.-A body which has freedom of the fourth order may be illustrated by the case of a rigid body, one point $P$ of which is constrained to a certain curve. The position of the body will then be specified by four quantities, viz., the arc of the curve from a fixed origin up to $P$, and three rotations about three axes intersecting in $P$. The reciprocal cylindroid will in this case assume an extreme form; it consists of screws. of zero pitch on all the normals to the curve at $P$.

$\S$ 139. Statics.-When a rigid body has freedom of the fourth order, the necessary and sufficient condition for equilibrium is, that the forces shall constitute a wrench upon a screw of the cylindroid reciprocal to the given screw complex. Thus, if one force can act on the body without disturbing equilibrium, then this force must lie on one of the two screws of zero pitch on the cylindroid. If there were no real screws of zero pitch on the cylindroid-that is, if the pitch conic were an ellipse, then it is impossible for equilibrium to subsist when a force acts. It is, however, worthy of remark, 
that if one force can act without disturbing the equilibrium, then another force (on the other screw of zero pitch) will be in the same predicament.

A couple which is in a plane perpendicular to the nodal line can be neutralized by the reaction of the constraints, and is, therefore, consistent with equilibrium. In no other case, however, can a body which has freedom of the fourth order be in equilibrium under the influence of a couple.

$\S 140$. Equilibrium of Five Forces. - The five forces must, if the body be free, belong to a screw complex of the fourth order. Draw the cylindroid reciprocal to the complex. The five forces must, therefore, intersect both the screws of zero pitch on the cylindroid. We, therefore, have the well-known condition that two straight lines can be drawn which intersect all the five forces. Four of the forces will determine the two lines, and therefore the fifth force may enjoy any liberty consistent with the requirement that it also intersects the two lines. This condition is also a sufficient one, so far as the positions of the forces are concerned.

If $A_{1}, \ldots A_{5}$ be the five forces, the ratio of $A_{1}: A_{2}$ is thus determined.

Let $P, Q$ be the two screws of zero pitch upon the cylindroid.

Let $X, Y$ be two screws reciprocal to $A_{1}, A_{2}$.

Let $Z$ be a screw reciprocal to $A_{3}, A_{4}, A_{5}$.

Construct the screw $I$ reciprocal to the five screws

$$
X, Y, P, Q, Z \text {. }
$$

Now, the four screws $X, Y, P, Q$ are reciprocal to the cylindroid $A_{1}, A_{2}$; therefore $I$, which is reciprocal to $X, Y, R, P, Q$, must lie upon the cylindroid $\left(A_{1}, A_{2}\right)$.

Since $P, Q, Z$ are all reciprocal to $A_{3}, A_{4} A_{5}$, it follows that $I$ being reciprocal to $P, Q, Z$ must belong to 
the screw complex $A_{3}, A_{4}, A_{5}$. Hence $I$ belongs to $\left(A_{1}, A_{2}\right)$, and also to $\left(A_{3}, A_{4}, A_{6}\right)$. If, therefore, forces along $A_{1}, \ldots A_{5}$ equilibrate, then the forces along $A_{1}, A_{2}$ must compound into a wrench on $I$. This condition determines the forces on $A_{1}, A_{2}$ (§ I 7 ).

$\S 14 \mathrm{I}$. Problem.-A free rigid body is acted upon by five forces: show how to move the body so that it shall not do work against nor receive energy from any one of the forces.

Let $A_{1}, \ldots A_{5}$ be the five forces. Draw two transversals $L, M$ intersecting $A_{1}, \ldots A_{4}$. Construct the cylindroid of which $L, M$ are the screws of zero pitch; find, upon this cylindroid, the screw $X$ reciprocal to $A_{5}$. Then the only movement which the body can receive, so as to fulfil the prescribed conditions, is a twist about the screw $X$. For $X$ is then reciprocal to $A_{1}, \ldots A_{5}$, and therefore a body only free to twist about $X$ will be unacted upon by any forces directed along $A_{1}, \ldots A_{5}$.

From the theory of reciprocal screws it follows that a body rotated around any of the lines $A_{1}, \ldots A_{5}$ will not do work against nor receive energy from a wrench on $X$.

As a particular case, if $A_{1}, \ldots A_{5}$ have a common transversal, then $X$ is that transversal, and its pitch is zero. In this case it is sufficiently obvious that $A_{1}, \ldots A_{5}$ cannot disturb the equilibrium of a body only free to rotate about $X$.

$\S$ 142. Impulsive Screws and Instantaneous Screws.-A body which is free to twist about all the screws of a screw complex of the fourth order receives an impulsive wrench on the screw $\eta$. It is required to calculate the co-ordinates of the screw $\theta$ about which the body will commence to twist, and also the initial reactions of the constraints.

Let $\lambda$ and $\mu$ be any two screws on the reciprocal 
cylindroid, then the reaction of the constraints may be considered to consist of wrenches on $\lambda, \mu$ of intensities $\lambda^{\prime \prime}, \mu^{\prime \prime}$. If we adopt the six absolute principal screws of inertîa as the screws of reference, then the body will commence to move as if it were free, but had been acted upon by a wrench of which the co-ordinates are proportional to $p_{1} \theta_{1}, \ldots, p_{6} \theta_{6}$. It follows that the given impulsive wrench, when compounded with the reactions of the constraints, must constitute the wrench of which the coordinates have been just written; whence if $h$ be a certain constant, we have the six equations-

$$
\begin{gathered}
h p_{1} \theta_{1}=\eta^{\prime \prime} \eta_{1}+\lambda^{\prime \prime} \lambda_{1}+\mu^{\prime \prime} \mu_{1}, \\
\& \text { c., \&c. } \\
h p_{6} \theta_{6}=\eta^{\prime \prime} \eta_{6}+\lambda^{\prime \prime} \lambda_{6}+\mu^{\prime \prime} \mu_{6} .
\end{gathered}
$$

Multiply the first of these equations by $\lambda_{1}$, the second by $\lambda_{2}$, \&c. : adding the six equations thus obtained, and observing that $\theta$ is reciprocal to $\lambda$, we have-

$$
\eta^{\prime \prime} \Sigma \eta_{1} \lambda_{1}+\lambda^{\prime \prime} \Sigma \lambda_{1}^{2}+\mu^{\prime \prime} \Sigma \lambda_{1} \mu_{1}=0,
$$

and similarly-

$$
\eta^{\prime \prime} \Sigma \eta_{1} \mu_{1}+\lambda^{\prime \prime} \Sigma \mu_{1} \lambda_{1}+\mu^{\prime \prime} \Sigma \mu_{1}^{2}=0 .
$$

From these two equations the unknown quantities $\lambda^{\prime \prime}, \mu^{\prime \prime}$ can be found, and thus the initial reaction of the constraints is known, substituting the values of $\lambda^{\prime \prime}, \mu^{\prime \prime}$ in the six original equations, the co-ordinates of the required screw $\theta$ are known.

$\S$ I 43. Principal Screws of Inertia.-We shall now show how the co-ordinates of the four principal screws of inertia belonging to the screw complex of the fourth order are to be computed. All the co-ordinates are, as before, referred to the six absolute principal screws of ineriia of the body $(\$ 105)$.

Let $\boldsymbol{\alpha}, \beta \gamma, \delta$ be any four co-reciprocal screws of the 
given screw complex. Then the co-ordinates of any other screw $\theta$ of the complex may be determined by-

$$
\begin{gathered}
\theta^{\prime \prime} \theta_{1}=a^{\prime \prime} a_{1}+\beta^{\prime \prime} \beta_{1}+\gamma^{\prime \prime} \gamma_{1}+\delta^{\prime \prime} \delta_{1}, \\
\& \text { c., \&c. } \\
\theta^{\prime \prime} \theta_{6}=a^{\prime \prime} a_{6}+\beta^{\prime \prime} \beta_{6}+\gamma^{\prime \prime} \gamma_{6}+\delta^{\prime \prime} \delta_{6} .
\end{gathered}
$$

We shall, as before, denote two screws on the reciprocal cylindroid by $\lambda, \mu$. If $\theta$ be a principal screw of inertia, then-

$$
\begin{gathered}
h p_{1}\left(a^{\prime \prime} a_{1}+\beta^{\prime \prime} \beta_{1}+\gamma^{\prime \prime} \gamma_{1}+\delta^{\prime \prime \prime} \delta_{1}\right)=a^{\prime \prime} a_{1}+\beta^{\prime \prime} \beta_{1}+\gamma^{\prime \prime} \gamma_{1}+\delta^{\prime \prime} \delta_{1} \\
+\lambda^{\prime \prime} \lambda_{1}+\mu^{\prime \prime} \mu_{1}, \\
\& c ., \& c . \\
h p_{6}\left(a^{\prime \prime} a_{6}+\beta^{\prime \prime} \beta_{6}+\gamma^{\prime \prime} \gamma_{6}+\delta^{\prime \prime} \delta_{6}\right)=a^{\prime \prime} a_{6}+\beta^{\prime \prime} \beta_{6}+\gamma^{\prime \prime} \gamma_{6}+\delta^{\prime \prime} \delta_{6} \\
+\lambda^{\prime \prime} \lambda_{6}+\mu^{\prime \prime} \mu_{6} .
\end{gathered}
$$

Multiplying the first of these equations by $a_{1}$, the next. by $a_{2}, \&$ c., adding the products, observing that $a$ is reciprocal to $\beta, \gamma, \delta, \lambda, \mu$, and repeating the operations for $\beta, \gamma, \delta$, we have the four equations-

$$
\begin{aligned}
& a^{\prime \prime}\left(\Sigma a_{1}{ }^{2}-h p_{a}\right)+\beta^{\prime \prime} \Sigma a_{1} \beta_{1} \quad+\gamma^{\prime \prime} \Sigma a_{1} \gamma_{1} \quad+\delta^{\prime \prime} \Sigma a_{1} \delta_{1} \quad=0, \\
& a^{\prime \prime} \Sigma \beta_{1} \alpha_{1}+\beta^{\prime \prime}\left(\Sigma \beta_{1}{ }^{2}-h p_{\beta}\right)+\gamma^{\prime \prime} \Sigma \beta_{1} \gamma^{\mathrm{T}}+\delta^{\prime \prime} \Sigma \beta_{1} \delta_{1}=0 \text {, } \\
& a^{\prime \prime} \Sigma \gamma_{1} a_{1}+\beta^{\prime \prime} \Sigma \gamma_{1} \beta_{1}+\gamma^{\prime \prime}\left(\Sigma \gamma_{1}{ }^{2}-h p \gamma\right)+\delta^{\prime \prime} \Sigma \gamma_{1} \delta_{1}=0 \text {, } \\
& a^{\prime \prime} \Sigma \delta_{1} \alpha_{1}+\beta^{\prime \prime} \Sigma \delta_{1} \beta_{1} \quad+\gamma^{\prime \prime} \Sigma \delta_{1} \gamma_{1} \quad+\delta^{\prime \prime}\left(\Sigma \delta_{1}{ }^{2}-h p_{\gamma}\right)=0 .
\end{aligned}
$$

From these four linear equations $a^{\prime \prime}, \beta^{\prime \prime}, \gamma^{\prime \prime}, \delta^{\prime \prime}$ can be eliminated, and we obtain an equation of the fourth degree for $h$. When $h$ is known, then $a^{\prime \prime}, \beta^{\prime \prime}, \gamma^{\prime \prime}, \delta^{\prime \prime}$ are known, and thus the co-ordinates of the four principal screws of inertia are determined.

$\S$ 144. Application of Euler's Theorem.-It may be of interest to show how the instantaneous screw corresponding to a given impulsive $\mathrm{sc}^{-r} \mathrm{w}$ can be deduced 
from Euler's theorem ( $\$ 64)$. If a body receive an impulsive wrench on a screw $\eta$ while the body is constrained to twist about a screw $\theta$, then we have seen in $\S 6$ I that the kinetic energy acquired is proportional to-

$$
\frac{\varpi^{2} \eta \theta}{u^{2} \theta}
$$

If $\theta_{1}, \theta_{3}, \theta_{3}, \theta_{4}$ be the co-ordinates of $\theta$ referred to the four principal screws of inertia belonging to the screw complex of the fourth order, then $(\$ \S 65,67)$ -

$$
\begin{aligned}
& \varpi_{\eta \theta^{2}}=\left(p_{1} \eta_{1} \theta_{1}+p_{3} \eta_{2} \theta_{2}+p_{3} \eta_{3} \theta_{3}+p_{4} \eta_{4} \theta_{4}\right)^{2}, \\
& u_{\theta}{ }^{2}=u_{1}^{2} \theta_{1}^{2}+u_{2}^{2} \theta_{2}^{2}+u_{3}^{2} u_{3}^{2}+u_{4}^{2} \theta_{4}^{2} .
\end{aligned}
$$

Hence we have to determine the four independent variables $\theta_{1}, \theta_{2}, \theta_{3}, \theta_{4}$, so that -

$$
\frac{\left(p_{1} \eta_{1} \theta_{1}+p_{2} \eta_{2} \theta_{2}+p_{3} \eta_{3} \theta_{3}+p_{4} \eta_{4} \theta_{4}\right)^{2}}{u_{1}^{2} \theta_{1}^{2}+u_{2}^{2} \theta_{2}^{2}+u_{3}^{2} \theta_{3}^{2}+u_{4}^{2} \theta_{4}^{2},}
$$

shall be a maximum. This is easily seen to be the case when $\theta_{1}, \theta_{2}, \theta_{3}, \theta_{4}$ are respectively proportional to-

$$
\frac{p_{1}}{u_{1}^{2}} \eta_{1}, \frac{p_{2}}{u_{2}^{2}} \eta_{2}, \frac{p_{3}}{u_{3}^{2}} \eta_{3}, \frac{p_{4}}{u_{4}^{2}} \eta_{4}
$$

This method might be applied to any order of freedom, and of course gives the same result as $\$ 67$.

$\S$ 145. General Remarks. - We shall here introduce some general reflections upon the problem of the determination of the instantaneous screw corresponding to a given impulsive screw. These reflections are called forth by the circumstance that for the freedom of the fourth order a different method of proceeding is required from that which has been used for the second and third orders.

It has been shown in $\S 53$ how the co-ordinates of the instantaneous screw corres 
pulsive screw can be determined when the rigid body is perfectly free. It will be observed that the connexion between the two screws depends only upon the three principal axes through the centre of inertia, and the radii of gyration about these axes. We may express this result more compactly by the familiar conception of the momental ellipsoid. The centre of the momental ellipsoid is at the centre of inertia of the rigid body, the directions of the principal axes of the ellipsoid are the same as the principal axes of inertia, and the lengths of the axes of the ellipsoid are inversely proportional to the corresponding radii of gyration. When, therefore, the impulsive screw is given, the momental ellipsoid alone must be capable of determining the corresponding instantaneous screw.

A family of rigid bodies may be conceived which have a common momental ellipsoid, every rigid body which fulfils nine conditions will belong to this family. If an impulsive wrench applied to a member of this family cause it to twist about a screw $\theta$, then the same impulsive wrench applied to any other member of the same family will cause it likewise to twist about $\theta$. If we added the further condition that the masses of all the members of the family were equal, then it would be found that the twist velocity, and the kinetic energy acquired in consequence of a given impulse, would be the same to whatever member of the family the impulse were applied (\$\$ 60, 6I).

$\S$ I 46. The Screw Complex of the $(n-1)$ o:der and Second Degree.-We shall denote a screw complex of the. $n^{\text {th }}$ order and first degree by $A$, and $\theta_{1}, \ldots \theta_{n}$ are the co-ordinates of a screw $\theta$ belonging to $A$, and referred to $n$ co-reciprocal screws chosen from $A$.

Let us first consider the interpretation of the linear equation between the $n$ co-ordinates of $\theta$ :- 


$$
a_{1} \theta_{1}+a_{2} \theta_{2}+\& c_{.}+a_{n} \theta_{n}=0
$$

All the screws whose co-ordinates satisfy this equation must be reciprocal to the screw $\xi$ belonging to $A$, of which the co-ordinates are proportional to-

$$
\frac{a_{1}}{p_{1}}, \ldots \frac{a_{n}}{p_{n}}
$$

hence all the screws whose co-ordinates satisfy the linear equation must be reciprocal to $7-n$ independent screws, viz., $\xi$ and $6-n$ screws from the screw complex reciprocal to $A$. Hence we have the following theorem $(\S 46)$.

If from a screw complex $(A)$ of the $n^{\text {th }}$ order and first degree, we select all the screws whose $n$ co-ordinates (when referred to $n$ screws of reference belonging to $A$ ) satisfy one linear equation, then the group of screws so selected constitute a screw complex of the $(n-1)^{\text {th }}$ order and first degree.

We shall now define a screw complex of the $(n-I)^{t h}$ order and second degeee.

If from a screw complex $A$ of the $n^{\text {th }}$ order and first degree, we select all the screws whose $n$ co-ordinates (when referred to $n$ screws of reference belonging to $A$ ) satisfy one homogeneous equation of the second degree, then the group of screws so selected constitute a screw complex of the $(n-\mathrm{I})^{\text {th }}$ order and second degree.

$\S$ I 47. Polar Screws.-Let $U=0$ denote a screw complex of the $(n-1)^{\text {th }}$ order and second degree, embraced within the screw complex of the $n^{\text {th }}$ order and first degree, which is denoted by $A$, then we define the polar of the screw $\theta$ with respect to $U_{\theta}=0$ to be the screw belonging to $A$, of which the $n$ co-ordinates are proportional to- 


$$
\frac{\mathbf{I}}{p_{1}} \frac{d U_{\theta}}{d \theta_{1}}, \ldots \frac{\mathbf{I}}{p_{n}} \frac{d U_{\theta}}{d \theta_{n}},
$$

it being understood that the $n$ screws of reference are coreciprocal.

If $n=6$, then $A$ consists of every screw in space, and the polar of $\theta$ is what we have already considered in § 9 r.

$\S$ I 48. Kinetic Complex.-We have seen $(\$ 67)$ that the kinetic energy of a body twisting about a screw $\theta$ belonging to a screw complex of the $n^{\text {th }}$ order and first degree, with a twist velocity $\frac{d \theta^{\prime}}{d t}$ is

$$
M\left(\frac{d \theta^{\prime}}{d t}\right)^{2}\left(u_{1}^{2} \theta_{1}^{2}+\ldots+u_{n}^{2} \theta_{n}^{2}\right),
$$

the screws of reference being the principal screws of inertia.

If we make $u_{1}^{2} \theta_{1}^{2}+\ldots+u_{n}^{2} \theta_{n}^{2}=0$, then $\theta$ must belong to a screw complex of the $(n-I)^{\text {th }}$ order and second degree. This complex is, of course, imaginary, for the kinetic energy of the body when twisting about any screw which belongs to it is zero. We may for convenience term this the kinetic screw complex.*

The polar $\eta$ of the screw $\theta$, with respect to the kinetic complex, has co-ordinates proportional to-

$$
\frac{u_{1}^{2}}{p_{1}} \theta_{1} \ldots, \frac{u_{n}^{2}}{p_{n}} \theta_{n}
$$

Comparing this with $\S 67$, we deduce the following important theorem :-

A quiescent rigid body is free to twist about all the srews of a screw complex $\mathrm{A}$. If the body receive an impulsive

* Dr. Klein has, in a letter to the writer, pointed out the importance of the kinetic complex. Dr. Klein was led to this complex by expressing the condition that the impulsive screw should be reciprocal to the corresponding instantaneous screw. 
wrench on a screw $\eta$ belonging to $\mathrm{A}$, then the body will commence to trwist about the screw $\theta$, of which $\eta$ is the polar with respect to the kinetic complex.

The screw $\eta$ is, of course, only one of a screw complex $S$ of the $(7-n)^{\text {th }}$ order, an impulsive wrench on any one of which would make a body commence to twist about $\theta(\$ 55) ; \eta$ is, however, the only screw belonging to $S$ which also belongs to $A$; a wrench on $\eta$ is the reduced wrench on $A$, appropriate to a wrench on any other screw belonging to $S(\S 66)$.

$\S$ I 49 The Potential Complex.-If a rigid body which has freedom of the $n^{\text {th }}$ order be displaced from a position of stable equilibrium by a twist of given amplitude about a screw $\theta$, of which the co-ordinates referred to the $n$ principal screws of the potential are $\theta_{1}, \ldots \theta_{n}$, then the potential energy of the new position is proportional to-

$$
v_{1}^{2} \theta_{1}^{2}+\& \text { c. }+v_{n}^{2} \theta_{n}^{2} \text {. }
$$

If this expression be equated to zero, it denotes a screw complex of the $(n-1)^{t h}$ order and second degree, which may be termed the potential complex.

The potential complex possesses a physical importance in every respect analogous to that of the kinetic complex; by reference to $(\S 72)$ the following theorem can be deduced.

If a rigid body be displaced from a position of equilibrium by a twist about a screw $\theta$, then a wrench acts upon the body in its new position on a screw which is the polar of $\theta$ with respect to the potential complex.

$\S 150$ Harmonic Screws. - The constructions by which the harmonic screws were determined in the case of the second and the third orders have no analogies in the fourth order. We shall, therefore, here state a general algebraical method by which they can be determined.

Let $U=0$ be the kinetic complex, and $V=0$ the 
potential complex, then it is well-known that one set of axes of reference can be found which will reduce both $U$ and $V$ to the sum of $n$ squares. These axes of reference are the harmonic screws.

We may here also make the remark, that any screw complex $U_{\theta}=0$ of the $(n-1)^{t h}$ order and second degree can always be transformed in one way to the sum of $n$ square terms with co-reciprocal screwes of reference; for if $U_{\theta}$ and $p_{\theta}=0$ be transformed so that each consists of the sum of $n$ square terms, then the form of the expression of $p_{\theta}(\S 40)$ shows that the screws are co-reciprocal. 


\section{CHAPTER XIII.}

THE DYNAMICS OF A RIGID BODY WHICH HAS FREEDOM OF THE FIFTH ORDER.

$\S 151$. Screw Reciprocal to Five Screws. - There is no more important theorem in the Theory of Screws than that which asserts the existence of one screw reciprocal to five given screws. At the commencement, therefore, of the Chapter of which this theorem is the foundation, it may be well to give a demonstration founded on elementary principles.

Let one of the five given screws be typified by

$$
\frac{x-x_{k}}{a_{k}}=\frac{y-y_{k}}{\beta_{k}}=\frac{z-z_{k}}{\gamma_{k}}\left(\text { pitch }=\rho_{k}\right),
$$

while the desired screw is defined by

$$
\frac{x-x_{1}^{\prime}}{a}=\frac{y-y^{\prime}}{\beta}=\frac{z-z^{\prime}}{\gamma}(\text { pitch }=\rho) .
$$

The condition of reciprocity $(\$ 22)$ produces five equations of the following type :-

$$
\begin{aligned}
& \boldsymbol{a}\left[\left(\rho+\rho_{k}\right) \boldsymbol{a}_{k}+\gamma_{k} y_{k}-\beta_{k} z_{k}\right]+\beta\left[\left(\rho+\rho_{k}\right) \beta_{k}+\boldsymbol{a}_{k} z_{k}-\gamma_{k} x_{k}\right] \\
+ & \gamma\left\lceil\left(\rho+\rho_{k}\right) \gamma_{k}+\beta_{k} x_{k}-\boldsymbol{a}_{k} y_{k}\right]+\boldsymbol{a}_{k}\left(\gamma y^{\prime}-\beta z^{\prime}\right)+\beta_{k}\left(\boldsymbol{\alpha} z^{\prime}-\gamma x^{\prime}\right) \\
+ & \gamma_{k}\left(\beta x^{\prime}-\boldsymbol{a} y^{\prime}\right)=0 .
\end{aligned}
$$

From these five equations the relative values of the six quantities

$$
a, \beta, \gamma, \gamma y^{\prime}-\beta z^{\prime}, \alpha z^{\prime}-\gamma x^{\prime}, \beta x^{\prime}-a y^{\prime}
$$

can be determined by linear solution. Introducing these values into the identity 


$$
a\left(\gamma y^{\prime}-\beta z^{\prime}\right)+\beta\left(a z^{\prime}-\gamma x^{\prime}\right)+\gamma\left(\beta x^{\prime}-a y^{\prime}\right)=0,
$$

gives the equation which determines $\rho$.

To express this equation concisely we introduce two classes of subsidiary magnitudes. We write one magnitude of each class as a determinant.

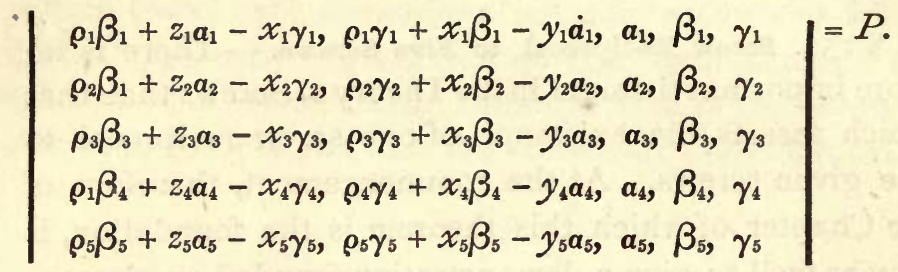

By cyclical interchange the two analogous functions $Q$ and $R$ are defined.

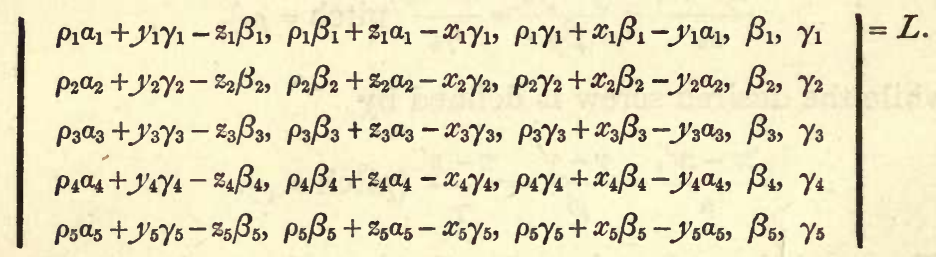

By cyclical interchange the two analogous functions $M$ and $N$ are defined.

The equation for $\rho$ reduces to

$$
\left(P^{2}+Q^{2}+R^{2}\right) \rho+P L+Q M+R N=0 .
$$

The reduction of this equation to the first degree is an independent proof of the principle, that one screw, and only one, can be determined which is reciprocal to five given screws; $\rho$ being known, $\boldsymbol{a}, \boldsymbol{\beta}, \boldsymbol{\gamma}$ can be found, and also two linear equations between $x^{\prime}, y^{\prime}, z^{\prime}$, whence the reciprocal screw is completely determined. 
$\S$ 152. Definition of the Sexiant. -When six screws, $A_{1}, \& c ., A_{6}$, are reciprocal to a single screw $T$, a certain relation must subsist between the six screws. This relation may be expressed by equating the determinant of $\S 4 \mathrm{I}$ to zero. The determinant (called the sexiant may be otherwise expressed as follows :-

The equations of the screw $A_{k}$ are

$$
\frac{x-x_{k}}{a_{k}}=\frac{y-y_{k}}{\beta_{k}}=\frac{z-z_{k}}{\gamma_{k}}\left(\text { pitch } \rho_{k}\right) \text {. }
$$

We shall presently show that we are justified in assuming for $T$ the equations

$$
\frac{x}{a}=\frac{y}{\beta}=\frac{z}{\gamma}(\text { pitch }=\rho) .
$$

The condition that $A_{k}$ and $T$ be reciprocal is

$$
\begin{gathered}
\left(\rho+\rho_{k}\right)\left(\boldsymbol{\alpha} \boldsymbol{a}_{k}+\beta \beta_{k}+\gamma \gamma_{k}\right)+x_{k}\left(\gamma \beta_{k}-\beta \gamma_{k}\right)+y_{k}\left(\boldsymbol{\alpha} \gamma_{k}-\gamma \boldsymbol{\alpha}_{k}\right) \\
+z_{k}\left(\beta \boldsymbol{a}_{k}-\boldsymbol{a} \beta_{k}\right)=0 .
\end{gathered}
$$

Writing the six equations of this type, found by giving $k$ the values 1 to 6 , and eliminating the six quantities

$$
\rho a, \rho \beta, \rho \gamma, a, \beta, \gamma,
$$

we obtain the result :-

$$
\begin{aligned}
& \left|a_{1} \rho_{1}+\gamma_{1} y_{1}-\beta_{1} z_{1}, \beta_{1} \rho_{1}+a_{1} z_{1}-\gamma_{1} x_{1}, \gamma_{1} \rho_{1}+\beta_{1} x_{1}-a_{1} y_{1}, a_{1}, \beta_{1}, \gamma_{1}\right|=0 \text {. } \\
& a_{2} \rho_{2}+\gamma_{2} y_{2}-\beta_{2} z_{2}, \beta_{2} \rho_{2}+a_{2} z_{2}-\gamma_{2} x_{2}, \gamma_{2} \rho_{2}+\beta_{2} x_{2}-a_{2} y_{2}, a_{3}, \beta_{2}, \gamma_{2} \\
& a_{3} \rho_{3}+\gamma_{3} y_{3}-\beta_{3} z_{3}, \beta_{3} \rho_{3}+a_{3} z_{3}-\gamma_{3} x_{3}, \gamma_{3} \rho_{3}+\beta_{3} x_{3}-a_{3} y_{3}, a_{3}, \beta_{3}, \gamma_{3} \\
& a_{4} \rho_{4}+\gamma_{4} y_{4}-\beta_{4} z_{4}, \beta_{4} \rho_{4}+a_{4} z_{4}-\gamma_{4} x_{4}, \gamma_{4} \rho_{4}+\beta_{4} x_{4}-a_{4} y_{4}, a_{4}, \beta_{4}, \gamma_{4} \\
& a_{5} \rho_{5}+\gamma_{5} y_{5}-\beta_{5} z_{5}, \beta_{5} \rho_{5}+a_{5} z_{5}-\gamma_{5} x_{5}, \gamma_{5} \rho_{5}+\beta_{5} x_{5}-\alpha_{5} y_{5}, \alpha_{5}, \beta_{5}, \gamma_{5} \\
& a_{6} \rho_{6}+\gamma_{6} y_{6}-\beta_{6} z_{6}, \beta_{6} \rho_{6}+a_{6} z_{6}-\gamma_{6} x_{6}, \gamma_{6} \rho_{6}+\beta_{6} x_{6}-a_{6} y_{6}, a_{6}, \beta_{6}, \gamma_{6}
\end{aligned}
$$

By transformation to any parallel axes the value of this determinant is unaltered. The evanescence of the determinant is therefore a necessary condition whenever the six screws are reciprocal to a single screw. Hence we 
sacrificed no generality in the assumption that $T$ passed through the origin.

Since the sexiant is linear in $x_{1}, y_{1}, z_{1}$, it appears that all parallel screws of given pitch reciprocal to one screw lie in a plane. Since the sexiant is linear in $\boldsymbol{a}_{1}, \beta_{1}, \gamma_{1}$, we have Möbius' theorem $(\S 80)$.

The property possessed by six screws when their sexiant vanishes may be enunciated in different ways, which are precisely equivalent.

(a). The six screws are all reciprocal to one screw.

(b). The six screws are members of a screw complex of the fifth order and first degree.

(c). Wrenches of appropriate intensities on the six. screws equilibrate, when applied to a free rigid body.

(d). Properly selected twist velocities about the six screws neutralize, when applied to a rigid body.

(e). A body might receive six small twists about the six screws, so that after the last twist the body would occupy the same position which it had before the first.

If seven wrenches equilibrate (or twists neutralize), then the intensity of each wrench (or the amplitude of each twist) is proportional to the sexiant of the six noncorresponding screws.

$\S$ 153. Equilibrium.-For a rigid body which has freedom of the fifth order to be in equilibrium, the necessary and sufficient condition is that the forces which act upon the body constitute a wrench upon that one screw to which the freedom is reciprocal. We thus see that it is not possible for a body which has freedom of the fifth order to be in equilibrium under the action of gravity unless the screw reciprocal to the freedom have zero pitch, and coincide in position with the vertical through the centre of inertia.

Professor Sylvester has shown* that when six lines, 
$P, Q, R, S, T, U$, are so situated that forces acting along them equilibrate when applied to a free rigid body, a certain determinant vanishes, and the six lines are in involution.*

Using the ideas and language of the Theory of Screws, this determinant is the sexiant of the six screws, the pitches of course being zero.

If $x_{m}, y_{m}, z_{m}$, be a point on one of the lines, the direction cosines of the same line being $\boldsymbol{a}_{m}, \boldsymbol{\beta}_{m}, \gamma_{m}$, the condition is

$\left|\begin{array}{llllll}\boldsymbol{a}_{1}, & \beta_{1}, & \gamma_{1}, & y_{1} \gamma_{1}-z_{3} \beta_{1}, & z_{1} a_{1}-x_{1} \gamma_{1}, & x_{1} \beta_{1}-y_{1} a_{1} \\ a_{2}, & \beta_{2}, & \gamma_{2}, & y_{2} \gamma_{2}-z_{2} \beta_{2}, & z_{2} a_{2}-x_{2} \gamma_{2}, & x_{2} \beta_{2}-y_{2} a_{2} \\ \boldsymbol{a}_{3}, & \beta_{3}, & \gamma_{3}, & y_{3} \gamma_{3}-z_{3} \beta_{3}, & z_{3} a_{3}-x_{3} \gamma_{3}, & x_{3} \beta_{3}-y_{3} a_{3} \\ \boldsymbol{a}_{4}, & \beta_{4}, & \gamma_{4}, & y_{4} \gamma_{4}-z_{4} \beta_{4}, & z_{4} a_{4}-x_{4} \gamma_{4}, & x_{4} \beta_{4}-y_{4} a_{4} \\ \boldsymbol{a}_{5}, & \beta_{5}, & \gamma_{5}, & y_{5} \gamma_{6}-z_{5} \beta_{5}, & z_{5} a_{5}-x_{5} \gamma_{5}, & x_{5} \beta_{5}-y_{5} a_{5} \\ \boldsymbol{a}_{6}, & \beta_{6}, & \gamma_{6}, & y_{6} \gamma_{6}-z_{6} \beta_{6}, & z_{6} a_{6}-x_{6} \gamma_{6}, & x_{6} \beta_{6}-y_{6} a_{6}\end{array}\right|=0$.

A single screw $X$ must be capable of being found which is reciprocal to all the six screws $P, Q, R, S, T, U$. Suppose the rigid body were only free to twist about $X$, then the six forces would not only collectively be in equilibrium, but severally would be unable to stir the body only free to twist about $X$.

In general a body able to twist about six screws (of any pitch) would have perfect freedom; but the body capable of rotating about each of the six lines, $P, Q, R, S, T, U$, which are in involution, is not necessarily perfectly free (Möbius).

* In the language of Plücker (Neue Geometrie des Raumes) a system of lines in involution forms a linear complex. In our language a system of lines in involution consists of the screws of equal pitch belonging to a screwe complex of the fifth order and first degree. See also Salmon's Geometry of Three Dimensions, third edition, p. 456 , note. It may save the reader some trouble to observe here that the word involution has been employed in a more generalised sense by Battaglini, and in quite a different sense by Klein. 
If a rigid body were perfectly free, then a wrench about any screw could move the body; if the body be only free to rotate about the six lines in involution, then a wrench about every screw (except $X$ ) can move it.

The conjugate axes of Professor Sylvester (p. 743) are presented in the present system as follows:-Draw any cylindroid which contains the reciprocal screw $X$, then the two screws of zero pitch on this cylindroid are a pair of conjugate axes. For a force on any transversal intersecting this pair of screws is reciprocal to the cylindroid, and is therefore in involution with the original system.

Draw any two cylindroids, each containing the reciprocal screw, then all the screws of the cylindroids form a screw complex of the third order. Therefore the two pairs of conjugate axes, being four screws of zero pitch, must lie upon the same quadric. This theorem is due to Professor Sylvester.

The cylindroid also presents in a clear manner the solution of the problem of finding two rotations which shall bring a body from one position to any other given position. Find the twist which would effect the desired change. Draw any cylindroid through the corresponding screw, then the two screws of zero pitch on the cylindroid are a pair of axes that fulfil the required conditions. If one of these axes were given the cylindroid would be defined and the other axis would be determinate.

$\S$ I54. Impulsive Screws and Instantaneous Screws.-We can determine the instantaneous screw corresponding to a given impulsive screw in the case of freedom of the fifth order by geometrical considerations. Let $\lambda$, as before, represent the screw reciprocal to the freedom, and let $\rho$ 'be the instantaneous screw which would correspond to $\lambda$ as an impulsive screw, if the body were perfectly free; let $\eta$ be the screw on which the body receives an 
impulsive wrench, and let $\xi$ be the screw about which the body would commence to twist in consequence of this impulse if it had been perfectly free.

The body when limited to the screw complex of the fifth order will commence to move as if it had been free, but had been acted upon by a certain unknown wrench on $\lambda$, together with the given wrench on $\eta$. The movement which the body actually acquires is a twisting motion about a screw $\theta$ which must lie on the cylindroid $(\xi, \rho)$. We therefore determine $\theta$ to be that one screw on the known cylindroid $(\xi, \rho)$ which is reciprocal to the given screw $\lambda$. The twist velocity of the initial twisting motion about $\theta$, as well as the intensity of the impulsive wrench on the screw $\lambda$ produced by the reaction of the constraints, are also determined by the same construction. For by $\S \mathrm{I} 7$ the relative twist velocities about $\theta, \xi$, and $\rho$ are known; but since $\eta^{\prime \prime}$ is known, the twist velocity about $\xi$ is known ( $(60)$; and therefore, the twist velocity about $\theta$ is known; finally, from the twist velocity about $\rho$, the intensity $\lambda^{\prime \prime}$ is determined.

$\S$ I 55. Analytical Investigation.-A quiescent rigid body which has freedom of the fifth order receives an impulsive wrench on a screw $\eta$ : it is required to determine the instantaneous screw $\theta$, about which the body will commence to twist.

Let $\lambda$ be the screw reciprocal to the freedom, and let the co-ordinates be referred to the absolute principal screws of inertia. The given wrench compounded with a certain wrench on $\lambda$ must constitute the wrench which, if the body were free, would make it twist about $\theta$, whence we deduce the six equations ( $h$ being an unknown quantity).

$$
\begin{gathered}
h p_{1} \theta_{1}=\eta^{\prime \prime} \eta_{1}+\lambda^{\prime \prime} \lambda_{1} \\
\& c ., \& c ., \\
h p_{6} \theta_{6}=\eta^{\prime \prime} \eta_{6}+\lambda^{\prime \prime} \lambda_{0} .
\end{gathered}
$$


Multiplying the first of these equations by $\lambda_{1}$, the second by $\lambda_{2}$, \&c., adding the six equations thus produced, and remembering that $\theta$ and $\lambda$ are reciprocal, we deduce

$$
\eta^{\prime \prime} \Sigma \eta_{1} \lambda_{1}+\lambda^{\prime \prime} \Sigma \lambda_{1}{ }^{2}=0
$$

This equation determines $\lambda^{\prime \prime}$ the intensity of the impulsive reaction of the constraints. The co-ordinates of the required screw $\theta$ are, therefore, proportional to the six quantities

$$
\frac{\eta_{1} \Sigma \lambda_{1}^{2}-\lambda_{1} \Sigma \eta_{1} \lambda_{1}}{p_{1}}, \& c ., \frac{\eta_{6} \Sigma \lambda_{1}{ }^{2}-\lambda_{6} \Sigma \eta_{1} \lambda_{1}}{p_{6}} .
$$

$\S$ I 56. Principal Screws of Inertia.-We are now enabled to determine the co-ordinates of the five principal screws of inertia; for if $\xi$ be a principal screw of inertia, then

whence

$$
h p_{1} \xi_{1}=\xi^{\prime \prime} \xi_{1}+\lambda^{\prime \prime} \lambda_{1},
$$

$$
\xi_{1}=\frac{\lambda^{\prime \prime} \lambda_{1}}{h p_{1}-\xi^{\prime \prime}}
$$

with similar values for $\xi_{2}$, \&c., $\xi_{6}$. Substituting these values in the equation

$$
p_{1} \lambda_{1} \xi_{1}+p_{2} \lambda_{2} \xi_{2}+p_{3} \lambda_{3} \xi_{3}+p_{4} \lambda_{4} \xi_{4}+p_{5} \lambda_{5} \xi_{5}+p_{6} \lambda_{6} \xi_{6}=0
$$

and making $\frac{\xi^{\prime \prime}}{h}=x$, we have for $x$ the equation

$$
\frac{p_{1} \lambda_{1}{ }^{2}}{p_{1}-x}+\frac{p_{2} \lambda_{2}{ }^{2}}{p_{2}-x}+\frac{p_{3} \lambda_{3}{ }^{2}}{p_{3}-x}+\frac{p_{4} \lambda_{4}{ }^{2}}{p_{4}-x}+\frac{p_{5} \lambda_{5}{ }^{2}}{p_{5}-x}+\frac{p_{6} \lambda_{6}{ }^{2}}{p_{6}-x}=0 .
$$

This equation is of the fifth degree, corresponding to the five principal screws of inertia. If $x^{\prime}$ denote one of the roots of the equation, then the corresponding principal screw of inertia has co-ordinates proportional to

$$
\frac{\lambda_{1}}{p_{1}-x^{\prime \prime}} \frac{\lambda_{2}}{p_{2}-x^{\prime}} \frac{\lambda_{3}}{p_{3}-x^{\prime \prime}}, \frac{\lambda_{4}}{p_{4}-x^{\prime}}, \frac{\lambda_{5}}{p_{5}-x^{\prime}}, \frac{\lambda_{8}}{p_{6}-x^{\prime}} .
$$


We may easily verify with these co-ordinates that each pair of principal screws of inertia are reciprocal : for let $x^{\prime}, x^{\prime \prime}$ be a pair of roots, then the difference between the two equations

$$
\Sigma \frac{p_{1} \lambda_{1}^{2}}{p_{1}-x^{\prime}}=0 \text { and } \Sigma \frac{p_{1} \lambda_{1}^{2}}{p_{1}-x^{\prime \prime}}=0 \text { is } \Sigma \frac{p_{1} \lambda^{2}}{\left(p_{1}-x^{\prime}\right)\left(p_{1}-x^{\prime \prime}\right)}=0 \text {; }
$$

but this is equally the condition that the two screws of which the co-ordinates are

$$
\frac{\lambda_{1}}{p_{1}-x^{\prime \prime}}, \& c ., \frac{\lambda_{1}}{p_{1}-x^{\prime \prime}}, \& c \text {. }
$$

shall be reciprocal ( $\$ 57)$.

It is also easy to see that

$$
\frac{x^{\prime \prime}}{x^{\prime \prime}-x^{\prime}} \Sigma \frac{p_{1} \lambda_{1}{ }^{2}}{p_{1}-x^{\prime}}+\frac{x^{\prime}}{x^{\prime}-x^{\prime \prime}} \Sigma \frac{p_{1} \lambda_{1}{ }^{2}}{p_{1}-x^{\prime \prime}}=\Sigma \frac{p_{1}{ }^{2} \lambda_{1}{ }^{2}}{\left(p_{1}-x^{\prime}\right)\left(p_{1}-x^{\prime \prime}\right)} \text {. }
$$

Since each of the terms on the left-hand side of this equation is zero, it follows that the right-hand side must be zero; but this is equivalent $(\S 54)$ to the statement that the principal screws of inertia are conjugate screws of inertia $(\S 57)$. 
I 70 )

\section{CHAPTER XIV.}

THE DYNAMICS OF A RIGID BODY WHICH HAS FREEDOM OF THE SIXTH ORDER.

$\S$ 157. Introduction.-When a rigid body has freedom of the sixth order, it is perfectly free. The screw complex of the sixth order includes every screw in space. That there is no reciprocal screw to such a complex is merely a different way of asserting the obvious proposition that when a body is perfectly free it cannot remain in equilibrium, if the forces which act upon it have a resultant.

$\S$ I 58. Impulsive screws.-Let $A_{1}, A_{2}$, \&c., denote a series of instantaneous screws which correspond respectively to the impulsive screws $R_{1}, R_{2}$, \&c., the body being perfectly free. Corresponding to each pair $A_{1}, R_{1}$ is a certain specific parameter. This parameter may be conveniently defined to be the twist velocity produced about $A_{1}$ by an impulsive wrench on $R_{1}$, of which the intensity is one unit. If six pairs, $A_{1} R_{1}, A_{2} R_{2}$, \&c., be known, and also the corresponding specific parameters, then the impulsive wrench on any other screw $R$ can be resolved into six impulsive wrenches on $R_{1}, \& \mathrm{c}$, $R_{6}$, these will produce six known twist velocities on $A_{1}, \&$ c., $A_{6}$, which being compounded together determine $A$, the twist velocity about $A$, and therefore the specific parameter of $R$ and $A$. We thus see that it is only necessary to be given six corresponding pairs, and their specific parameters, in order to determine completely the effect of any other impulsive wrench.

We are now going to show that if seven pars $o$ 
corresponding instantaneous and impulsive screws be given, then the relation between every other pair is absolutely determined. It appears from $\S 30$ that appropriate twist velocities about $A_{1}, \&$ c., $A_{7}$ can neutralise. When this is the case, the corresponding impulsive wrenches on $R_{1}$, \&c., $R_{7}$, must equilibrate, and therefore the relative values of the intensities are known. It follows that the specific parameter of each pair $A_{1}, R_{1}$ is proportional to the quotient obtained by dividing the sexiant of $A_{2}, \&$ c., $A_{6}$, by the sexiant of $R_{2}, \&$ c., $R_{6}$. With, therefore, the exception of a constant factor, the specific parameter of every pair of screws is known, when seven corresponding screws are known.

When therefore seven instantaneous screws are known, and the corresponding seven impulsive screws, we are enabled by geometrical construction alone to deduce the instantaneous screw corresponding to any eighth impulsive screw, and vice versâ.

A precisely similar similar method of proof will give us the following theorem:-

If a rigid body be in position of stable equilibrium under the influence of a sytem of forces which have a potential, and if the twists abont seven given screws evoke wrenches about seven other given screws, then, without knowing any further about the forces, we shall be able to determine the screw on which a wrench is evoked by a twist about any eighth screw.

We shall state the results of the present section in a form, which may, perhaps, interest the student of modern geometry. We must conceive two corresponding systems of screws, of which the correspondence is completely established, when, to any seven screws regarded as belonging to one system, the seven corresponding screws in the other system are known. To every screw in space viewed as belonging to one system will corres- 
pond another screw viewed as belonging to the other system. Six screws can be found, each of which coincides with its correspondent. To a screw complex of the $n^{\text {th }}$ order and $m^{\text {th }}$ degree in one system will correspond a screw complex of the $n^{\text {th }}$ order and $m^{\text {th }}$ degree in the other system.

We add here a few examples to illustrate the use which may be made of screw co-ordinates.

$\S$ I 59. Theorem.-When an impulsive force acts upon a free quiescent rigid body, the directions of the force and of the instantaneous screw are parallel to a pair of conjugate diameters in the momental ellipsoid.

Let $\eta_{1}, \ldots \eta_{6}$ be the co-ordinates of the force referred to the absolute principal screws of inertia, then $(\S 37)$

$$
\left(\eta_{1}+\eta_{2}\right)^{2}+\left(\eta_{3}+\eta_{4}\right)^{2}+\left(\eta_{5}+\eta_{6}\right)^{2}=1,
$$

and from $(\S 93)$ it follows that the direction cosines of $\eta$ with respect to the principal axes through the centre of inertia are

$$
\left(\eta_{1}+\eta_{2}\right),\left(\eta_{3}+\eta_{4}\right),\left(\eta_{5}+\eta_{6}\right) .
$$

If $a, b, c$ be the radii of gyration, then the instantaneous screw corresponding to $\eta$ has for co-ordinates

$$
+\frac{\eta_{1}}{a},-\frac{\eta_{2}}{a},+\frac{\eta_{3}}{b},-\frac{\eta_{4}}{b},+\frac{\eta_{5}}{c},-\frac{\eta_{6}}{c} \text {. }
$$

The condition that $\eta$ and its instantaneous screw shall be parallel to a pair of conjugate diameters of the momental ellipsoid is

$$
a^{2}\left(\eta_{1}+\eta_{2}\right) \frac{\eta_{1}-\eta_{2}}{a}+b^{2}\left(\eta_{3}+\eta_{4}\right) \frac{\eta_{3}-\eta_{4}}{b}+c^{2}\left(\eta_{5}-\eta_{6}\right) \frac{\eta_{5}-\eta_{6}}{c}=0 ;
$$

or

$$
\Sigma p_{1} \eta_{1}^{2}=p_{\eta}=0 .
$$

But if the impulsive wrench on $\eta$ be a force, then the pitch of $\eta$ is zero, whence the theorem is proved. 
$\S$ I60. Theorem.-When an impulsive wrench acting on a free rigid body produces an instantaneous rotation, the axis of the rotation must be perpendicular to the impulsive screw.

Let $\eta_{1}, \ldots \eta_{6}$ be the axis of the rotation, then

$$
\Sigma p_{1} \eta_{1}^{2}=0
$$

or

$a\left(\boldsymbol{\eta}_{1}-\boldsymbol{\eta}_{2}\right)\left(\boldsymbol{\eta}_{1}+\eta_{2}\right)+b\left(\boldsymbol{\eta}_{3}-\boldsymbol{\eta}_{4}\right)\left(\boldsymbol{\eta}_{3}+\eta_{4}\right)+c\left(\boldsymbol{\eta}_{5}-\boldsymbol{\eta}_{6}\right)\left(\boldsymbol{\eta}_{5}+\boldsymbol{\eta}_{6}\right)=0$,

whence the screw of which the co-ordinates are $+a \eta_{1}$, $-a \eta_{2},+b \eta_{3}, \& c$., is perpendicular to $\eta$, and the theorem is proved.

From this theorem, and the last, we infer that, when an impulsive force acting on a rigid body produces an instantaneous rotation, the direction of the force, and the axis of the rotation, are parallel to the principal axes of a section of the momental ellipsoid.

$\$$ I6r. Principal Axis.-If $\eta$ be a principal axis of a rigid body, it is required to prove that

$$
\Sigma p_{1}^{3} \eta_{1}^{2}=0,
$$

reference being made to the absolute principal screws of inertia.

For in this case a force along a line $\theta$ intersecting $\eta$, compounded with a couple in a plane perpendicular to $\eta$, must constitute an impulsive wrench to which $\eta$ corresponds as an instantaneous screw, whence we deduce ( $(93), h$ and $k$ being arbitrary constants.

$$
\begin{gathered}
\theta_{1}=\frac{h}{p_{1}} \frac{d R}{d \eta_{1}}+k p_{1} \eta_{1}, \\
\& \mathrm{c} .
\end{gathered}
$$

Expressing the condition that $p^{\theta}=0$, we have 


$$
k^{2} \Sigma p_{1}{ }^{3} \eta_{1}{ }^{2}+2 h k \Sigma p_{1} \eta_{1} \frac{d R}{d \eta_{1}}+h^{2} \Sigma \frac{\mathrm{I}}{p_{1}}\left(\frac{d R}{d \eta_{1}}\right)^{2}=0 ;
$$

but we have already seen $(\$ \$ 93$, I05) that the two last terms of this equation are zero, whence the required theorem is demonstrated.

The formula we have just proved may be written in the form

$$
\Sigma p_{1} \cdot p_{1} \eta_{1} \cdot p_{1} \eta_{1}=0
$$

This shows that if the body were free, then an impulsive force suitably placed would make the body commence to rotate about $\eta$. Whence we have the following theorem :-

A rigid body previously in unconstrained equilibrium in free space is supposed to be set in motion by a single impulsive force; if the initial axis of twist velocity be a principal axis of the body, the initial motion is a pure rotation, and conversely. (Mr. Townsend, Educational Times, reprint, Vol. xxi., p. I07.)

It may also be asked what is the point of the body one of the three principal axes through which coincides with $\eta$ ? This point is the intersection of $\theta$ and $\eta$. To determine the co-ordinates of $\theta$ it is only necessary to find the relation between $h$ and $k$, and this is obtained by expressing the condition that $\theta$ is reciprocal to $\eta$, whence we deduce

$$
2 h+k u_{\eta}^{2}=0 .
$$

Thus $\theta$ is known, and the required point is determined. If the body be fixed at this point, and then receive the impulsive couple perpendicular to $\eta$, the instantaneous reaction of the point will be directed along $\theta$.

$\S$ I62. Harmonic Screws.-We shall conclude by stating for the sixth order the results which are included as particular cases of the general theorems in Chapter VIII. 
If a perfectly free rigid body be in equilibrium under the influence of a system of forces as restricted in $\S 6$, then six screws can be found such that each pair are conjugate screws of inertia, as well as conjugate screws of the potential, and these six screws are called harmonic screws. If the body be displaced from its position of equilibrium by a twist of small amplitude about a harmonic screw, and if the body further receive a small initial twisting motion about the same screw, then the body will continue for ever to perform small twist oscillations about that screw. And, more generally, whatever be the initial circumstances, the movement of the body is compounded of twist oscillations about the six harmonic screws. 
271

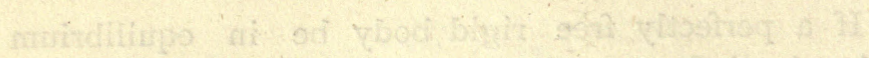

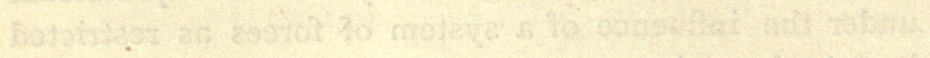

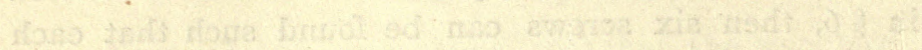

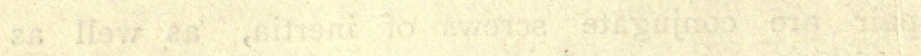

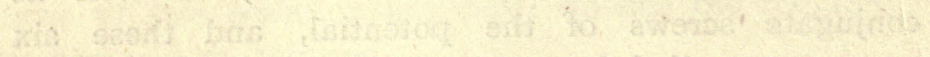

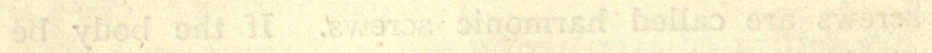

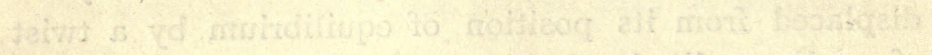

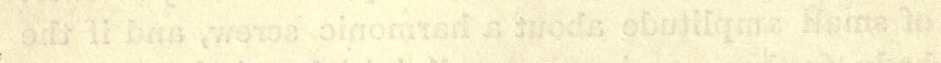

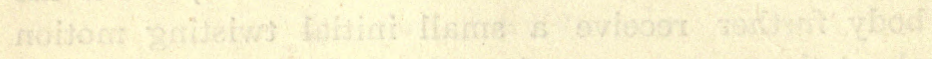

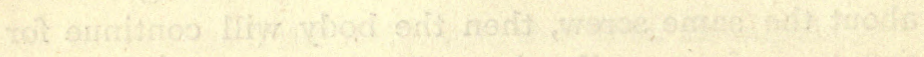

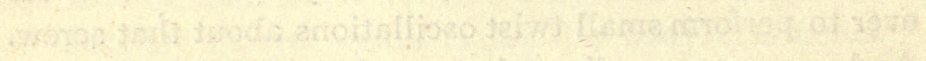

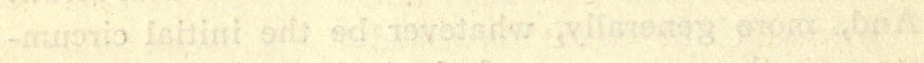

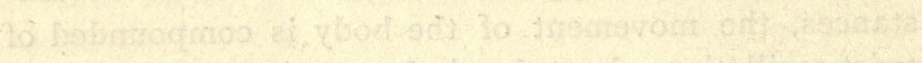

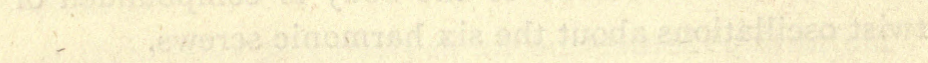




\title{
A P P E N D I X.
}

\author{
No. I.
}

I HERE briefly describe the principal works known to me which bear on the subject of the present volume.

Pornsot (L.)-Sur la composition des moments et la composition des aires (1804). Paris Journal de l'Ecole Polytechnique ; t. vi. 13 cah., pp. 182-205 (1806).

In this paper the author of the conception of the couple, and of the laws of composition of couples, has demonstrated the important theorem that any system of forces applied to a rigid body can be reduced to a single force, and a couple in a plane perpendicular to the force.

Chasles (M.)-Note sur les propriëtés générales du système de deux corps semblables entr'eux et placés d'une manière quelconque dans l'espace; et sur le déplacement fini ou infiniment petit d'un corps solide libre. Bulletin des Sciences Mathématiques, par Ferussac. Vol. xiv., pp. 321-326 (Paris, 1830).

The author shows that there always exists one straight line, about which it is only necessary to rotate one of the bodies to place it similarly to the other. Whence (p. 324) he is led to the following fundamental theorem :-

I'on peut toujours transporter un corps solide libre d'une position dans une autre position quelconque, déterminée par le mouvement continu d'une vis à laquelle ce corps serait fixé invariablement. 
That this theorem is really due to Chasles there can be little doubt. He explicitly claims it in note 34 to the Aperçu Historique. Three or four years later than the paper we have cited, Poinsot published his celebrated "Théorie Nouvelle de la Rotation des Corps" (Paris, 1834). In this he enunciates the same theorem. As Poinsot does not refer to Chasles, I had been led, in ignorance of Chasles' previous paper, to attribute the theorem to Poinsot (Transactions of Royal Irish Academy, Vol. xxv., p. 160); but I corrected the mistake in Phil. Trans., I874, p. 16.

\section{MöвIUs (A. F.)-Lehrbuch der Statik (Leipzig, I 837).}

This book is, we learn from the preface, one of the numerous productions to which the labours of Poinsot has given rise. The first part, pp. I-355, discusses the laws of equilibrium of forces, which act upon a single rigid body. The second part, pp. 1-313, discusses the equilibrium of forces acting upon several rigid bodies connected together. The characteristic feature of the book is its great generality. I here enunciate some of the principal theorems.

If a number of forces acting upon a free rigid body be in equilibrium, and if a straight line of arbitrary length and position be assumed, then the algebraic sum of the tetrahedra, of which the straight line and each of the forces in succession are pairs of opposite edges, is equal to zero (p. 94).

If four forces are in equilibrium they must be generators of the same hyperboloid (p. 177).

If five forces be in equilibrium they must intersect two common straight lines (p. r 79).

If the lines of action of five forces be given, then a certain plane $S$ through any point $P$ is determined. If the five forces can be equilibrated by one force through $P$, then this one force must lie in $S$ (p. 180).

To adopt the notation of Professor Cayley, we denote by 12 the perpendicular distance between two lines I, 2, multiplied into the sine of the angle between them (Comptes Rendus, t. lxi., pp. 829-830). Möbius shows (p. 189) that if forces 
along four lines 1, 2, 3, 4 equilibrate, the intensities of these forces are proportional to

$$
\sqrt{23 \cdot 24 \cdot 14}, \sqrt{13 \cdot 14 \cdot 34}, \sqrt{12 \cdot 14 \cdot 24}, \sqrt{12 \cdot 13 \cdot 23} .
$$

It is also shown that the product of the forces on $\mathrm{I}$ and 2 , multiplied by 12 , equals the product of the forces on 3 and 4 multiplied by 34. He hence deduces Chasles' theorem (Liouville's Journal, t. xii., p. 222), that the volume of the tetrahedron formed by two of the forces is equal to that formed by the remaining two.

MöBIUS (A. F.)-Ueber die Zusammensetzung unendlich kleiner Drehungen. Crelle's Journal ; t. 18, pp. 189-212 (Berlin, $1838)$.

This memoir contains many very interesting theorems, of which the following are the principal:-Any given displacement of a rigid body can be effected by two rotations. Two equal parallel and opposite rotations compound into a translation. Rotations about intersecting axes are compounded like forces, If a number of forces acting upon a free body make equilibrium, then the final effect of a number of rotations (proportional to the forces) on the same axes will be zero. If a body can be rotated about six independent axes, it can have any movement whatever.

RODRIGUES (O.)-Des lois géométriques qui régissent les déplacements d'un système solide dans l'espace et de la variation des co-ordonnées, provenant de ces déplacements considérés indépendamment des causes qui peuvent les produire. Liouville's Journal; t. 5, pp. $380-440$ (5th Dec., 1840).

This paper consists mainly of elaborate formulæ relating to displacements of finite magnitude. It has been already cited for an important remark ( $(12)$. 
Chasles (M.)-Propriétés géométriques rélatives au mouvement infiniment petit d'un corps solide libre dans l'espace. Comptes Rendus; t. xvi., pp. 1420-1432 (1843).

A pair of "droites conjuguées" are two lines by rotations about which a given displacement can be communicated to a rigid body. Two pairs of "droites conjuguées" are always generators of the same hyperboloid.

Pornsot (L.)-Théorie nouvelle de la rotation des corps. Liouville's Journal ; t. xvi., pp. 9-129, 289-336 (March, 1851).

This is Poinsot's classical memoir, which contains his beautiful geometrical theory of the rotation of a rigid body about $a$ fixed point. In a less developed form the Theory had been previously published in Paris in 1834 , as already mentioned.

CAyley (A.)-On a new analytical representation of curves in space Quarterly Mathematical Journal; Vol. iii., pp. 225-236 (1860): Vol. v., pp. 8I-86.

In this paper the conception of the six co-ordinates of a line is introduced for the first time.

Sylvester (J. J.)-Sur l'involution des lignes-droites dans l'espace, considérées comme des axes de rotation. Comptes Rendus; t. lii., pp. 741-746 (April, 186I).

Any small displacement of a rigid body can generally be represented by rotations about six axes (Möbius). But this is not the case if forces can be found which equilibrate when acting along the six axes on a rigid body. The six axes in this case are in involution. The paper discusses the geometrical features of such a system, and shows, when five axes are given, how the locus of the sixth is to be found. Möbius had shown that through any point a plane of lines can be drawn in involution with five given lines. The present paper shows how the plane can be constructed. All the transversals intersecting a pair of conjrgate axes are in involution with five given lines. Any two pairs of conjugate axes lie on the same hyperboloid. 
Two forces can be found on any pair of conjugate axes, which are statically equivalent to two given forces on any other given pair of conjugate axes. In presenting this paper M. Chasles remarks that Mr. Sylvester's results lead to the following construction:-Conceive that a rigid body receives any small displacement, then lines drawn tnrough any six points of the body perpendicular to their trajectories are in involution. M. Chasles takes occasion to mention also some other properties of the conjugate axes.

Sylvester (J. J.)-Note sur l'involution de six lignes dans l'espace.

Comptes Rendus; t. lii., pp. 815-817 (April, r86r).

The six lines are $1,2,3,4,5,6$. Let the line $i$ be represented by the equations

$$
\begin{aligned}
& a_{i} x+b_{i} y+c_{i} z+d_{i} u=0, \\
& a_{i} x+\beta_{i} y+\gamma_{i} z+\delta_{i} u=0,
\end{aligned}
$$

and let $i, j$ represent the determinant

$$
\begin{array}{llll}
a_{i} & b_{i} & c_{i} & d_{i} \\
a_{i} & \beta_{i} & \gamma_{i} & \delta_{i} \\
a_{j} & b_{j} & c_{j} & d_{j} \\
a_{j} & \beta_{j} & \gamma_{j} & \delta_{j}
\end{array}
$$

Form now the determinant $\Delta_{6}-$

\begin{tabular}{|llllll} 
& I, 2 & I, 3 & I, 4 & I, 5 & I, 6 \\
$2, I$ & & 2,3 & 2,4 & 2,5 & 2,6 \\
$3, I$ & 3,2 & & 3,4 & 3,5 & 3,6 \\
$4, I$ & 4,2 & 4,3 & & 4,5 & 4,6 \\
$5, I$ & 5,2 & 5,3 & 5,4 & & 5,0 \\
6,1 & 6,2 & 6,3 & 6,4 & 6,5 &
\end{tabular} \mid

If $\Delta_{6}=0$, the lines are in involution. Considering only the figures $1,2,3,4,5$, the determinant $\Delta_{s}$ can be formed. If 
$\Delta_{\mathbf{6}}=0$ and $\Delta_{\mathbf{0}}=0$, the five lines $1,2,3,4,5$ are in involution. If all the other minors are zero, the six lines will intersect a single transversal. If $\Delta_{5}=0$, without any other condition, the five lines $\mathrm{I}, \mathbf{2}, \mathbf{3}, 4,5$ intersect a single transversal. If $\Delta_{4}=0$ without any other condition, the lines I, 2, 3, 4 have but one common transversal (Cayley). A determinant can be found which is equal to the square root of $\Delta_{6}$. This square root is the determinant given in $\S 153$.

Grassmann (H.)-Die Ausdehnungslehre. Berlin (1862).

A system of $n$, numerically equal, "Grössen erster Stufe," of which each pair are " normal," is discussed on p. I13. A set of co-reciprocal screws is a particular case of this very general conception.

The "inneren Produkte" of two "Grössen" divided by the product of their numerical values, is the cosine of the angle between the two "Grössen." If $a, b, c, \ldots$ be normal, and if $k, l$ be any two other "Grössen," then

$$
\cos \angle k l=\cos \angle a k \cos \angle a l+\cos \angle b k . \cos \angle b l,+\& c \text {. (p. 139). }
$$

Here we have a very general theory, which includes screw co-ordinates as a particular case.

In a note on p. 222 the author states that the displacement of a body in space, or a general system of forces, form an "allgemeine räumliche Grösse zweiter Stufe."

The "kombinatorische Produkt" (p. $4 \mathrm{I}$ ) of $n$ screws will contain as a factor that single function whose evanescence would express that the $n$ screws belonged to a screw complex of the $(n-1)^{\text {th }}$ order.

Plücker (J.)-On a new geometry of space. Phil. Trans., 1865. Vol. 155, pp. 725-791.

In this paper the linear complex is defined ( $p$. 733). Some applications to optics are made (p. 760); the six co-ordinates of a line are considered (p. 774); and the applications to the geometry of forces (p. 786). 
PLüCKRR (J.) - Fundamental views regarding mechanics. Phil. Trans. (1866), Vol. 156, pp. 361-380.

The object of this paper is to "connect, in mechanics, translatory and rotatory movements with each other by a principle in geometry analogous to that of reciprocity." One of the principal theorems is thus enunciated:- "Any number of rotatory forces acting simultaneously, the co-ordinates of the resulting rotatory force, if there is such a force, if there is not, the co-ordinates of the resulting rotatory dyname, are obtained by adding the co-ordinates of the given rotatory forces. In the case of equilibrium the six sums obtained are equal to zero."

Mannheim (A.)-Sur le déplacement d'un corps solide. Journal de Mathématiques, $2^{\circ}$ Series, t. xl. (1866).

To M. Mannheim belongs the credit of having been the first to study geometrically the kinematics of a constrained rigid body from a perfectly general point of view. This paper contains the following theorem :-

When a rigid body has freedom of the second order, any point of the body must be displaced on a certain surface, and at any instant all the normals to these surfaces will intersect two straight lines.

This is easily seen from the Theory of Screws, because any force reciprocal to the cylindroid expressing this freedom must be a normal to all the surfaces belonging to the points on it.

Spottiswoode (W.)-Note sur l'équilibre des forces dans l'espace.

Comptes Rendus; t. Ixvi., pp. 97-103 (January, 1868).

If $P_{o}, \&$ c., $P_{n-1}$ be $n$ forces in equilibrium, and if $(0,1)$ denote the moment of $P_{o}, P_{1}$, then the author proves* that

$$
\begin{array}{r}
P_{1}(0,1)+P_{1}(0,2)+\& c_{0}=0 \\
P(1,0)+P_{2}(1,2)+\& c_{0}=0 \\
P(2,0)+P_{1}(2,1)+\ldots=0
\end{array}
$$

- We may remark that since the moment of two lines is the virtual co-efficient of two screws of zero pitch, these equations are given at once by virtual velocities, if we rotate the body round each of the forces in succession. 
As we have thus $n$ equations to determine only the relative values of $n$ quantities, the redundancy is taken advantage of to prove that

$$
\frac{P_{0}^{2}}{[0,0]}=\frac{P_{1}^{2}}{[1,1]}=\& c
$$

where $[0,0],[1,1], \& c$. , are the coefficients of $(0,0),(1,1)$, $\&$ c., in the determinant

$$
\left|\begin{array}{ccc}
(0,0) & (0,1) & \ldots \\
(1,0) & (1,1) & \ldots \\
. & .
\end{array}\right|
$$

When the number of forces is less than seven, it is shown how the formulæ admit of a special transformation, which expresses the conditions to be fulfilled.

This very elegant result may receive an extended interpretation. If $P_{o}, P_{1}, P_{2}, \&$ c., denote the intensities of wrenches on the screws $0,1,2, \&$ c. ; and if (12) denote the virtual co-efficient of I and 2, then, when the formulæ of Mr. Spottiswoode are satisfied, the $n$ wrenches equilibrate, provided that the screws belong to a screw complex of the $(n-1)^{\text {th }}$ order and first degree.

Plücker (J.)-Neue Geometrie des Raumes gegründet auf die Betrachtung der geraden Linie als Raumelement. Leipzig (B. G. Trübner, 1868-69), pp. 1-374.

This elaborate work is the principal authority on the theory of the linear complex. The subject is essentially geometrical, but there are a few remarks on mechanics; thus the author, on p. 24, introduces the word "Dyname:"-“Durch den Ausdruck 'Dyname,' habe ich die Ursache einer beliebigen Bewegung eines starren Systems, oder, da sich die Natur dieser Ursache, wie die Natur einer Kraft überhaupt, unserem Erkennungsvermögen entzieht, die Bewegung selbst: statt der Ursache die Wirkung, bezeichnet." Although it is not very easy to see the precise meaning of this passage, yet it appears that a 'Dyname' 
may be either a twist or a wrench (to use the language of the Theory of Screws.)

On p. 25 we read:- "Dann entschwindet das specifisch Mechanische, und, um mich auf eine kurze Andeutung zu beschränken : es treten geometrische Gebilde auf, welche zu Dynamen in derselben Beziehung stehen, wie gerade Linien zu Kräften und Rotationen." There can be little doubt that the "geometrische Gebilde," to which Plücker refers, are what we have called screws.

As we have already stated $(\S 16)$, it is in this book that we find the first mention of the surface which we call the cylindroid.

Through any point a cone of the second degree can be drawn, the generators of which are lines belonging to a linear complex of the second degree. If the point be limited to a certain surface the cone breaks up into two planes. This surface is of the fourth class and fourth degree, and is known as Kummer's surface, or as the surface of singularities appropriate to the given linear complex. (See Kummer, Abhandl. der Berl. Akad., 1866). This theory is of interest for our purpose, because the locus of screws reciprocal to a cylindroid is a very special linear complex of the second degree, of which the cylindroid itself is the surface of singularities.

KLEIN (Felix).-Zur Theorie der Linien-Complexe des ersten und zweiten Grades. Math. Ann., II. Band, pp. 198-226 (14th June, 1869).

The "simultaneous invariant" of two linear complexes is discussed. In our language this function is the virtual coefficient of the two screws reciprocal to the complexes. The six fundamental complexes are considered at length, and many remarkable geometrical properties proved. It is a matter of no little interest that these purely geometrical researches have a physical significance attached to them by the Theory of Screws.

This paper also contains the following proposition:- If $x_{1}, \ldots, x_{6}$ be the co-ordinates of a line, and $k_{1}, \ldots k_{6}$ be con- 
stants, then the family of linear complexes denoted by

$$
\frac{x_{1}^{2}}{k_{1}-\lambda}+\& \text { c. }+\frac{x_{0}^{2}}{k_{6}-\lambda}=0 \text {, }
$$

have a common surface of singularities where $\lambda$ is a variable parameter. If the roots $\lambda_{1}$, \&c. be known, we have a set of quasi elliptical co-ordinates for the line $x$. (Compare with § 156 ).

It is in this memoir that we find the enunciation of the remarkable geometrical principle which, when transformed into the language and conceptions of the Theory of Screws, asserts the existence of one screw reciprocal to five given screws.

KLEIN (F.) - Die allgemeine lineare Transformation der LinienCo-ordinaten. Math. Ann., Vol. ii., p. 366-371 (August 4, 1869).

Let $U_{1}, \ldots U_{\mathrm{b}}$ denote six linear complexes. The moments of a straight line, with its conjugate polars with respect to $U_{1}, \ldots U_{0}$, are, when multiplied by certain constants, the homogeneous co-ordinates of the straight line, and are denoted by $x_{1}, \ldots x_{8}$. Arbitrary values of $x_{1}, \&$ c., do not denote a straight line, unless a homogeneous function of the second degree vanishes.* If this condition be not satisfied, then a linear complex is defined by the co-ordinates, and the function is called the invariant of the linear complex. The simultaneous invariant of two linear complexes is a function of the co-ordinates, or is equal to $\Delta \sin \phi$ $-\left(K+K^{\prime}\right) \cos \phi$, where $K$ and $K^{\prime}$ are the parameters of the linear complexes, $\Delta$ the perpendicular distance, and $\phi$ the angle between their principal axes. If this quantity be zero, the two linear complexes are in involution. (The reader will observe that the word involution is here employed in a very different sense to that in which the same word is used by Professor Sylvester.)

The co-ordinates of a linear complex are the simultaneous

- This equation expresses that the pitch of the screw denoted by the co-ordinates is zero. 
invariants of the linear complex with each of six given linear complexes multiplied by certain constants. The six linear complexes can be chosen so that each one is in involution with the remaining five. The reader will easily perceive the equivalent theorems in the Theory of Screws:

\section{Zeuthen (H. G.)-Notes sur un système de co-ordonnées linéaire dans l'espace. Math. Ann., Vol. i., pp. 432-454 (1869).}

The co-ordinates of a line are the components of an unit force on the line decomposed along the six edges of a tetrahedron. These co-ordinates must satisfy one condition, which expresses that six forces along the edges of a tetrahedron have a single resultant force. The author makes applications to the theory of the linear complex.

Regarding the six edges as screws of zero pitch, they are not co-reciprocal. It may, however, be of interest to show how these co-ordinates may be used for a different purpose from that for which the author now quoted has used them. Call the virtual co-efficients of the opposite pairs of edges $L, M, N$. If the co-ordinates of a screw with respect to this system be $\theta_{1} \ldots \theta_{0}$, then the pitch is

$$
\frac{1}{2}\left(L \theta_{1} \theta_{2}+M \theta_{3} \theta_{4}+N \theta_{3} \theta_{\theta}\right)
$$

and the virtual co-efficient of the two screws $\phi, \theta$ is

$$
L\left(\theta_{1} \phi_{2}+\theta_{2} \phi_{1}\right)+M\left(\theta_{2} \phi_{6}+\theta_{4} \phi_{2}^{\prime}\right)+N\left(\theta_{3} \phi_{6}+\theta_{6} \phi_{3}\right) .
$$

Battaglini (G.)-Memoria sulle dinami in involuzione. Atti di Napoli IV. (1869).

The co-ordin ates of a dyname are the six forces which acting along the edges of a tetrahedron, are equivalent to the dyname. This memoir investigates the properties of dynames of which the co-ordinates satisfy one or more linear equations. The author shows analytically the existence of two associated systems of dynames such that all the dynames of the first order are correlated to all the dynames of the second. These correspond to what we would call two reciprocal screw complexes. 
Battaglini (G.) -Sul movimento geometrico infinitesimo di un sistemo rigido.

Estratto dal Rendiconto della $R$. Accademia delle Seiense fisiche e Matematiche. (Fascicolo, May 5, 1870).

This paper and the last belong to a series by the same author, in which the tetrahedron co-ordinates are employed in the analytical development of the statics of a rigid body, as well as the theory of small displacements.

Mannheim (A.)-Etude sur le déplacement d'une figure de forme invariable. Recueil des Memoires, des Savants étrangers; t. xx. Journal de l'école Polytechnique, cah. 43, pp. 57-122 (1870).

This paper discusses the trajectories of the different points of a body when its movement takes place under prescribed conditions. Had I been sooner acquainted with this paper, I should have attributed to M. Mannheim the theorem about the screws of zero pitch on a cylindroid given in $\S 95$. Another theorem of the same class is also given by M. Mannheim. When a rigid body has freedom of the third order, then for any point on the surface of a certain quadric* the possible displacements are limited to a plane.

BALL (R. S.)-On the small oscillations of a Rigid Body about a fixed point under the action of any forces, and, more particularly, when gravity is the only force acting. Transactions of the Royal Irish Academy, Vol. xxiv., pp. 593-627 (January 24, 1870.)

The principal theorems contained in this paper are demonstrated in $\S \mathrm{I} 30$ of the present volume.

* The reader will easily see that this is the pitch quadric. 
KLEIN (Felix).-Notiz betreffend den Zusammenhang der LinienGeometrie mit der Mechanik starrer Körper. Math. Ann., Vol. iv., pp. 403-415 (June, 1871).

Among many interesting matters this paper contains the germ of the physical conception of reciprocal screws. We thus read on p. 413:- "Es lässt sich nun in der That' ein physikalischer Zusammenhang zwischen Kräftesystemen und unendlich kleinen Bewegungen angeben, welcher es erklärt, wie so die beiden Dinge mathematisch co-ordinirt auftreten. Diese Beziehung ist nicht von der Art, dass sie jedem Kräftesystem eine einzelne unendlich kleine Bewegung zuordnet, sondern sie ist von anderer Art, sie ist eine dualistische.

“Es sei ein Kräftesystem mit de丸 Coordinaten $\Xi, H, Z, \Lambda$, $M, N$, und eine unendlich kleine Bewegung mit den Coordinaten $\Xi^{\prime}, H^{\prime}, Z^{\prime}, \Lambda^{\prime}, M, N$ gegeben, wobei man die Co-ordinaten in der im $\S 2$ besprochenen Weise absolut bestimmt haben mag. Dann repräsentirt, wie hier nicht weiter nachgewiesen werden soll, der Ausdruck

$$
\Lambda^{\prime} \Xi+M^{\prime} N+N^{\prime} Z+\Xi^{\prime} \Lambda+H^{\prime} M+Z^{\prime} N
$$

das Quantum von Arbeit, welches das gegebene Kräftesystem bei Eintritt der gegebenen unendlich kleinen Bewegung leistet. Ist insbesondere

$$
\Lambda^{\prime} \Xi+M^{\prime} H+N^{\prime} Z+\Xi^{\prime} \Lambda+H^{\prime} M+Z^{\prime} N=0,
$$

so leistet das gegebene Kräftesystem bei Eintritt der gegebenen unendlich kleinen Bewegung keine Arbeit. Diese Gleichung nun repräsentirt uns, indem wir einmal $\Xi, H, Z, \Lambda, M, N$, das andere $\Xi^{\prime}, H^{\prime}, Z^{\prime}, \Lambda^{\prime}, M^{\prime}, N^{\prime}$ als veränderlich betrachten, den Zusammenhang zwischen Kräftesystemen und unendlich kleinen Bewegungen."

KLEIN (Felix).-Ueber gewisse in der Linien-Geometrie auftretende Differential-Gleichungen. Math. Ann., V. Band, pp. 278 -303 (November, 1871).

There is a remarkable invariant of $n$ linear complexes $U_{1}=0$, $U_{2}=0, \ldots U_{n}=0$. For let $\lambda_{1}, \ldots \lambda_{n}$ be arbitrary multipliers, 
then $\lambda_{1} U_{1}+\ldots+\lambda_{n} U_{n}=0$ also denotes a linear complex, provided that a certain condition is satisfied. This condition is presented as a homogeneous function of the second degree in $\lambda_{1}, \ldots \lambda_{n}$ equated to zero. The discriminant of the function is the invariant in question.

BALL (R. S.)-The Theory of Screws-a geometrical study of the kinematics, equilibrium, and small oscillations of a Rigid Body. Transactions of the Royal Irish Academy, Vol. xxv., pp. 137-217 (November 13, 1871).

This is the original paper on the Theory of Screws. In estimating how far the contents of this paper are novel, it is to be remembered that the cylindroid had been discussed by Plücker two or three years previously, while the conception of reciprocal screws had been announced by Klein a few months before. Both these authors would, of course, have been referred to in this paper had I been acquainted with their works at the time the paper was written.

Clifford (W. K.) - On Biquaternions. Proceedings of the London Mathematical Society, Nos. 64, 65, p. 382 (1 2th June, 1873).

A Biquaternion is defined to be the ratio of two "motors." A "motor" may be said to bear the same relation to the dyname of Plücker which a vector bears to a linear magnitude. The Biquaternions are shown to be intimately associated with the speculations of the geometry of elliptic space. See Klein's wonderful paper, "Ueber die nicht Euclidische Geometrie." Math. Ann., Band IV., pp. 573-625.

BALI (R. S.)-Researches in the Dynamics of a Rigid Body by the aid of the Theory of Screws (June 19, 1873). Philosophical Transactions, pp. i5-40 (1874).

The $n$ principal screws of inertia belonging to a rigid body which has freedom of the $n^{\text {th }}$ order are here discussed. 
LiNDEMANN (F.)-Ueber unendlich kleine Bewegungen und über Kräflesysteme bei allgemeiner projectivischer Massbestimmung. Math. Ann., 7th Vol., pp. 56-143 (July, 1873).

This is a memoir upon the statics and kinematics of a rigid body in elliptic or hyperbolic space. Among several results closely related to the Theory of Screws, we find that the cylindroid is only the degraded form in parabolic or common space of a surface of the fourth order, with two double lines.

\section{WEILER (A.)-Ueber die verschiedenen Gattungen der Complexe} zweiten Grades. Math. Ann., Vol. vii., pp. 145-207 (July, 1873).

In this elaborate memoir the author enumerates fifty-eight different species of linear complex of the second order. The classification is based upon Kummer's surface, which defines the singularities of the screw complex.

BALL, (R. S.)-Screw Co-ordinates and their applications to problems in the Dynamics of a Rigid Body. Transactions of the Royal Irish Academy, Vol. xxv., pp. 259-327 (January 12, 1874).

To trace a satisfactory connexion between an impulsive screw and the corresponding instantaneous screw is the principal object of this paper. It is here shown that to the instantaneous screw, whose co-ordinates are $\theta_{1}, \ldots, \theta_{6}$, corresponds an impulsive screw, whose co-ordinates are proportional to $p_{1} \theta_{1}, \ldots, p_{6} \theta_{6}$, reference being made to the absolute principal screws of inertia.

Everetr (J. D.)-On a new method in Statics and Kinematics. (Part I.) Messenger of Mathematics. New Series. No. 39 (1874).

This paper contains applications of quaternions. The operator $w+V_{\sigma}(\quad)$ is a " motor," $w$ and $\sigma$ being vectors, the former denoting a translation or couple, the latter a rotation or force. The pitch is $S \frac{\sigma}{\sigma}$. The equation to the central axis is $\rho=V \frac{\pi}{\sigma}$ $-x \sigma$. The work done in a small motion is $-S \varpi_{1} \sigma_{2}-S \varpi_{2} \sigma_{1}$ 
The existence of $k$ equations of the first degree between $n$ motors is the condition of their belonging to a screw complex of the first degree, and of order $n-k$.

EveretT (J. D.)-On a new method in Statics and Kinematics. (Part II.) Messenger of Mathematics. New Series. No. 45 (1875).

This paper contains further developments of the theory of linear relations between motors. Several of the leading theorems in screws are directly deduced from motor equations by the ordinary rules of determinants.

Everetr (J. D.)-On a new method in Statics and Kinematics. (Part III.) Messenger of Mathematics. New Series. No. 53 (1875).

This paper is devoted to the operation of motors upon motors. The interpretation of such operations is given, the laws of operation are laid down, and some applications are made, involving the use of a special symbol called a "motor determinant." 


\section{No. II.}

\section{NOTE ON THE CYLINDROID.}

As the study of surfaces is greatly facilitated by accurate models, I here give the details of the construction of the model of the cylindroid figured in the frontispiece. A boxwood cylinder, ${ }^{m}{ }^{m} 5$ long and $0^{m} \cdot{ }_{5}$ diameter, is chucked to the mandril of a lathe furnished with a dividing plate. A drill is mounted on the slide rest, and driven by overhead gear. The parameter $p_{\alpha}-p_{\beta}$ (in the present case $0^{m} .066$ ) is divided into one hundred parts. By the screw, which moves the slide rest parallel to the bed of the lathe, the drill can be moved to any number $z$ of these parts from its original position at the centre of the length of the cylinder. Four holes are to be drilled for each value of $z$. These consist of two pairs of diametrically opposite holes. The directions of the holes intersect the axis of the cylinder at right angles. The following table will enable the work to be executed with facility. $l$ is the angle of $\S_{15}$ :-

\begin{tabular}{|c|c|c|c|c|}
\hline$z$ & $l$ & $90-l$ & $180+l$ & $270-l$ \\
\hline 0.0 & 0 & 90 & 180 & 70 \\
\hline 17.4 & 5 & 85 & 185 & 265 \\
34.2 & 10 & 80 & 190 & 260 \\
50.0 & 15 & 75 & 195 & 255 \\
64.3 & 20 & 70 & 200 & 250 \\
76.5 & 25 & 65 & 205 & 245 \\
86.6 & 30 & 60 & 210 & 240 \\
94.0 & 35 & 55 & 215 & 235 \\
98.5 & 40 & 50 & 220 & 230 \\
$100 \cdot 0$ & 45 & 45 & 225 & 225 \\
\hline
\end{tabular}


For example, when the slide has been moved $34^{\circ} \mathbf{2}$ parts from the centre of the cylinder, the dividing plate is to be set successively to $10^{\circ}, 80^{\circ}, 190^{\circ}, 260^{\circ}$, and a hole is to be drilled in at each of these positions. The slide rest is then to be moved on to $5^{\circ}$ parts, and holes are to be drilled in at $15^{\circ}, 75^{\circ}, 195^{\circ}$, $255^{\circ}$. Steel wires, each about $0^{m} \cdot 3$ long, are to be forced into the holes thus made, and half the surface is formed. The remaining half can be similarly constructed : a length of $0^{m} \cdot 066$ $\cos 2 l$ is to be coloured upon each wire to show the pitch. The sign of the pitch is indicated by using one colour for positive, and another colour for negative pitches.

THE END. 



U. C. BERKELEY LIBRARIES

C061354097
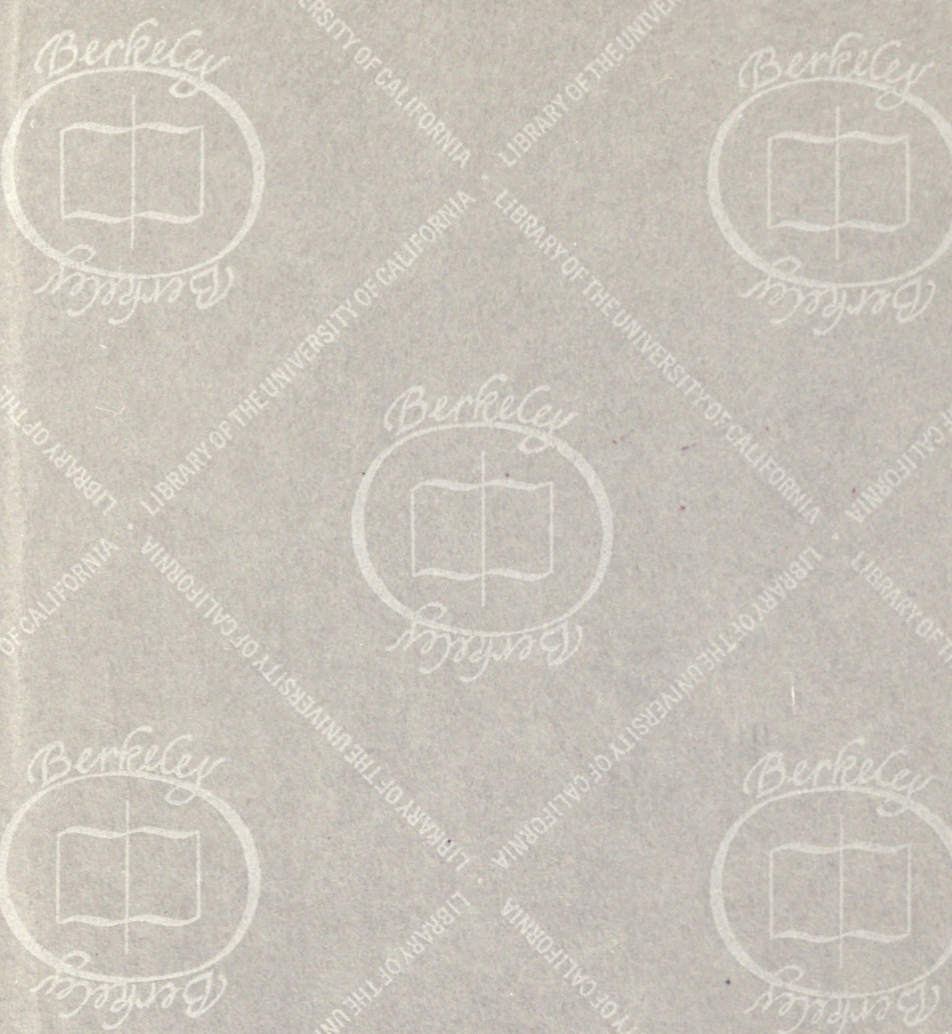
